\title{
Spinning black hole binary dynamics, scattering amplitudes, and effective field theory
}

\author{
Zvi Bern, ${ }^{1}$ Andres Luna, ${ }^{1}$ Radu Roiban, ${ }^{2}$ Chia-Hsien Shen, ${ }^{1}$ and Mao Zeng ${ }^{3}$ \\ ${ }^{1}$ Mani L. Bhaumik Institute for Theoretical Physics, Department of Physics and Astronomy, \\ UCLA, Los Angeles, California 90095, USA \\ ${ }^{2}$ Institute for Gravitation and the Cosmos, Pennsylvania State University, \\ University Park, Pennsylvania 16802, USA \\ ${ }^{3}$ Institute for Theoretical Physics, ETH Zürich, 8093 Zürich, Switzerland
}

(Received 6 April 2021; accepted 28 April 2021; published 17 September 2021)

\begin{abstract}
We describe a systematic framework for finding the conservative potential of compact binary systems with spin based on scattering amplitudes of particles of arbitrary spin and effective field theory. An arbitrary-spin formalism is generally required in the classical limit. By matching the tree and one-loop amplitudes of four spinning particles with those of a suitably chosen effective field theory, we obtain the $\operatorname{spin}_{1}-\operatorname{spin}_{2}$ terms of a two-body effective Hamiltonian through $\mathcal{O}\left(G^{2}\right)$ and valid to all orders in velocity. Solving Hamilton's equations yields the impulse and spin changes of the individual bodies. We write them in a surprisingly compact form as appropriate derivatives of the eikonal phase obtained from the amplitude. It seems likely this structure persists to higher orders. We also point out various double-copy relations for general spin.
\end{abstract}

DOI: 10.1103/PhysRevD.104.065014

\section{INTRODUCTION}

\section{A. Overview}

The landmark detection of gravitational waves by the LIGO and Virgo Collaborations [1] has opened a new window into the universe. The promise of major new discoveries calls for an invigorated effort to develop new theoretical tools for predictions of gravitational-wave signals matching the precision of current and future observations. Current predictions for gravitational-wave signals are based on a variety of complementary theoretical approaches. This includes the effective one-body (EOB) formalism [2], numerical relativity [3], and self-force formalisms [4]. In the inspiral phase, we have the traditional post-Newtonian (PN) approximation using methods in classical gravity [5,6] and the nonrelativistic general relativity (NRGR) formalism [7,8] based on effective field theory (EFT), as well as the post-Minkowskian (PM) expansion [9-17]. The various approaches provide important nontrivial confirmation and information in overlap regions of the PN, PM, and self-force expansions $[15,18,19]$. For recent reviews see Refs. [20,21].

Published by the American Physical Society under the terms of the Creative Commons Attribution 4.0 International license. Further distribution of this work must maintain attribution to the author(s) and the published article's title, journal citation, and DOI. Funded by SCOAP .
In recent years the post-Minkowskian approach, which is a relativistic weak-field expansion in Newton's constant, has risen in prominence because, at fixed order in Newton's constant, it naturally yields the exact velocity dependence of observable quantities. These properties mirror those of scattering amplitudes, which are fundamental building blocks of observables in quantum field theory. Combining techniques in scattering amplitudes and EFT, effective Hamiltonians have been derived in Refs. [12,22,23] that straightforwardly determine classical dynamics of bound orbits via their equations of motion. The usefulness of this framework has recently been demonstrated through the construction of the conservative two-body Hamiltonian at the third order in Newton's constant expansion [14,15]. Such Hamiltonians can be imported into the EOB framework $[10,16]$ used for gravitational-wave template construction. An important feature of results obtained along these lines is that they have a much simpler analytic structure than those obtained in other approaches, on the one hand, because the velocity expansion is resummed and, on the other, because scattering amplitudes naturally eliminate certain gauge-redundant structures that would generically appear.

Amplitude-based methods leverage powerful techniques that have been developed over the years for computing quantum scattering amplitudes in gauge and gravity theories (for reviews see e.g., Refs. [24,25]). The basic philosophy is to focus on gauge-invariant quantities that can be recursively computed from simpler building blocks: 
on-shell recursion relations [26] allow us to build more complex tree-level amplitudes directly from lower-point ones, and the modern unitarity method [27-31] then assembles tree amplitudes into integral representations of loop amplitudes. Because there is a close link between classical physics and quantum scattering amplitudes (see e.g., [10-15,22,23,32-40]), advanced methods for finding the latter can also be applied to solving certain nontrivial classical gravitational problems. The Kawai-Lewellen-Tye (KLT) [41] and Bern-Carrasco-Johansson (BCJ) [25,42,43] double-copy relations give gravitational scattering amplitudes directly in terms of much simpler gauge-theory ones, enabling explicit (super)gravity calculations at remarkably high orders of perturbation theory [44,45]. Massless and massive helicity methods $[46,47]$ have proven to be especially effective for calculating four-dimensional amplitudes. These tools have already demonstrated their utility for calculations of interest in gravitational-wave physics.

In this paper we focus on spin-dependent classical interactions of binary systems, in the post-Minkowskian expansion. As highlighted by the recent detection of black hole spin during inspiral phase [48], such effects are of considerable importance in light of astrophysical evidence that black holes can have a variety of intrinsic angular momenta, including close to maximally allowed values [49]. The presence of spin can lead to qualitative changes in the dynamics of a binary system, such as the orbitalplane precession when the spins are not aligned with the orbital angular momentum (see e.g., Ref. [50]). Such an effect would lead, in particular, to a modulation of the amplitude, frequency, and phase of the observed gravitational wave signal.

Inclusion of spin effects in the post-Newtonian expansion has a long history in a variety of frameworks [51-57]. The effect of spin in the context of the PN approximation has also been considered using elementary-particle scattering amplitudes [23,34]. Reference [23] further extracts PN potentials via EFT techniques and provides an early indication of the correspondence between minimal coupling and Kerr black holes. The analogous problem in the post-Minkowskian framework, where all orders in velocity are kept, has been comparatively less explored. For the problem of two Kerr black holes scattering, Ref. [58] derived a solution at linear order in Newton's constant with the full spin dependence using traditional methods and derived a corresponding two-body Hamiltonian. This was later shown to be equivalent to minimal amplitudes in massive spinor helicity formalism [59-62], and these amplitudes at order $G$ were used to derive a two-body effective Hamiltonian [63]. Physical observables with spins can also be extracted directly from scattering amplitudes [38], as demonstrated at order $G$ in [61]. At order $G^{2}$, a complete all-orders-in-velocity spin-orbit Hamiltonian is known [64]. Beyond linear order in spin, only partial results are available to all orders in velocity [37,59-62,65-67].

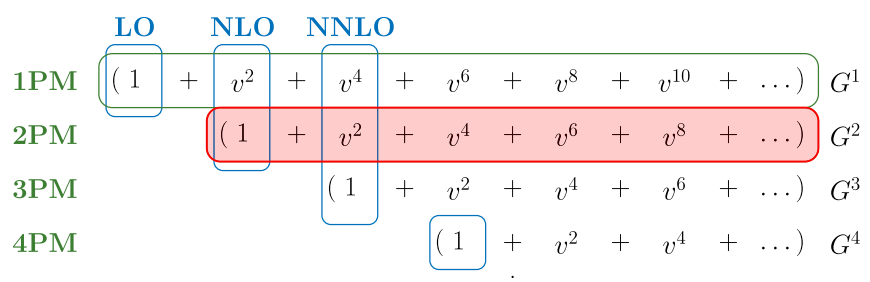

FIG. 1. The previously known results in PN and PM expansions of the (bilinear in spin) $\operatorname{spin}_{1}-$ spin $_{2}$ interactions in the two-body potential are outlined in horizontal (green) and vertical (blue) directions, respectively. The new results in this paper at $\mathcal{O}\left(G^{2}\right)$ and all orders in velocity correspond to the shaded (red) region. Each horizontal row corresponds to the same order in $G$, or the PM expansion. The velocity expansion is indicated by $v^{n}$. Each vertical column corresponds to the same PN order for the $\operatorname{spin}_{1}-\operatorname{spin}_{2}$ interaction, where the leading order (LO), next-toleading order (NLO), next-to-next leading order (NNLO), and the static part at $G^{4}$ are known up to quadratic in spins.

The table in Fig. 1 shows the status of the $\operatorname{spin}_{1}-\operatorname{spin}_{2}$ interactions analyzed in some detail in this paper at $\mathcal{O}\left(G^{2}\right)$, indicating the previously known terms in both the velocity and the $G$ expansions as well the new results.

\section{B. Summary of paper}

In this paper, we aim to answer several important questions in the amplitude-based approach. First, conventional field theory considers elementary spins $[23,34,66]$. The results are a priori not necessarily the same as those obtained with continuous classical spins. Using massivespinor-helicity formalism, the universality of spindependent effects was shown at linear in $G$ order and partially at order $G^{2}[59-62,67]$. Bootstrapping results for Kerr black holes from the massive-spinor-helicity method, however, is known to have ambiguities beyond quartic order in spins at $G^{2}[37,59,60,62]$, as new spin-multipole moments are allowed. It would be desirable to have a complementary formulation, with arbitrary spins, that can generate amplitudes from first principles. Second, for applications to LIGO and VIRGO, it is crucial to extract quantities of interest for the bound-state problem in a format that can be straightforwardly compared with previously known results. In the post-Minkowskian scenario without spins, there are several methods for doing so available $[10,12,14,15,39]$. In the presence of spins, however, the known results from EFT or scattering angles are limited either to leading order in $G$ or to special configurations of spins $[23,38,59,61,63]$. The goal of this paper is to build a systematic framework bridging the gaps between quantum scattering amplitudes, classical gravity, and bound orbits for spinning objects. A key part is to identify a new direct link between the scattering amplitudes including spin and physical observables, via the eikonal phase $[68,69]$.

A central component of our paper is an amplitude-based formalism for incorporating spinning effects for binary 
systems in a post-Minkowskian framework, i.e., fixed order in $G$ and all orders in velocity. Our results are new in several directions. First we construct a field theory with arbitrary-spin particles, smoothly interpolating from elementary particles to classical spinning particles. Next, we formulate an EFT for spinning particles. The amplitudes from this arbitrary-spin field theory are translated, through EFT matching, into an effective potential which can be used to study bound-state problems. Conversely, the formalism can also turn any classical Hamiltonians into gauge-invariant scattering amplitudes, allowing for a straightforward comparison of the gauge-invariant content of two Hamiltonians. The usefulness of our setup is demonstrated through a new result for spin-dependent effects: we obtain a two-body Hamiltonian that describes the interactions linear in the spin of each body, referred to as "spin ${ }_{1}-$ spin $_{2}$ " or "bilinear in spin," through order $G^{2}$ and to all orders in velocity. Finally, as discussed in more detail in Ref. [70], we use this Hamiltonian to calculate physical observables - the momentum and spin transfer-via the classical equations of motion, and organize them into an eikonal-based formula, providing a direct link between scattering amplitudes and classical observables. All results presented here are for generic spin orientation. We now summarize each section in turn.

In Sec. II, we begin with a basic introduction to classical spins, including the formulation of an arbitrary-spin field theory following the path of Refs. [71,72]. The arbitraryspin formalism constructed here is a natural framework for capturing higher powers of spin interaction; this may be contrasted with the more familiar spin- $1 / 2$ or spin- 1 cases that could be used to extract low-order spin interactions $[23,34,66]$. Nonminimal interactions at linear order in $G$ are characterized in Sec. III and are similar to those in the world-line formulation [52] of spinning particles given in Ref. [57]. At linear order in $G$, the Lagrangian may be interpreted as the covariantization of the most general parity-even gravitational form factor. Our stress tensor reproduces the all-orders-in-spin stress tensor at order $G$ of Ref. [58]. For the stress tensor, or equivalently the onshell two-matter-one-graviton vertex, we also present new double-copy relations to all orders in the spin for arbitrary nonminimal coupling, expressing the complete set of gravitational interactions in terms of gauge-theory ones. Double-copy properties in the context of gravitational waves have been discussed recently in Refs. [14,15,60,73].

In Secs. IV and V, we compute various tree-level and one-loop amplitudes with higher-spin particles. At the relatively low order considered in this paper, we will not need the full arsenal of amplitude techniques that become important at higher orders. We therefore use polarization tensors to incorporate the spin degrees of freedom. and make only modest use of the double copy [25,41,42] to write compact expressions for amplitudes. We first obtain the two-to-two scattering of higher-spin particles at tree level, truncated to bilinear order in spins. Then we calculate the tree-level gravitational Compton amplitude, and find simple KLT-like relations. In Sec. V, we use the Compton amplitude to extract the required contribution to the fourpoint one-loop amplitude of two distinct spinning particles. We then reduce the integrand to a basis of scalar integrals using the massive extension [74] of Forde's formalism [31]. This formalism clarifies the connection of basis integral coefficients and integrands and efficiently extracts the needed contribution in the classical limit.

In Sec. VI, we construct an EFT for spinning objects, following the path of Ref. [12] in the spinless case and Ref. [23] in the spin case under the PN framework. This EFT allows us to map scattering amplitudes to effective Hamiltonians, which can then be straightforwardly applied to bound-orbit problems. We classify spin interactions explicitly to bilinear-in-spin order. The on-shell matching scheme reduces the number of independent operators. We show how to compute scattering amplitudes in this EFT. We also point out the crucial role of the $S O(3)$ algebra of classical spins in order to obtain results for generic spin orientation. Combining with the one-loop amplitudes obtained in Sec. V, we derive the bilinear in spin Hamiltonian through order $G^{2}$ and to all orders in velocity.

In Sec. VII, we obtain the momentum and spin change in the scattering regime starting from our derived classical Hamiltonian. The three-dimensional nature of the scattering process makes the construction of the perturbative solution of the equations of motion somewhat more involved than for the case of spinless particles. An alternative approach is to directly obtain observables from the amplitudes [13,38], bypassing the Hamiltonian and EFT matching. A very interesting question is whether there exist, in general, simple and direct relations between physical quantities and suitably defined finite parts of amplitudes scattering amplitudes analogous to the one for the spinless or aligned spin case $[10,15,39,59]$. The ability to do this for spin [38] suggests that this might be more generally possible. We indeed find such a relation for generic spin, generalizing the eikonal formula $[68,69]$ to the case with spin, obtaining not only the impulse, but also the spin kick from appropriate derivatives of the eikonal phase. This striking result suggests that it should be possible to develop much more streamlined formalisms for extracting physical observables from scattering amplitudes at higher orders. We leave the details to a forthcoming paper [70].

In order to ensure the reliability of our results, we perform a number of nontrivial checks for the $\mathcal{O}\left(G^{2}\right)$ contributions to the interactions bilinear in spin. This includes comparison with the post-Newtonian NLO spinorbit results of Refs. [23,54], the NNLO results of Ref. [55] in the overlapping region to $\mathcal{O}\left(G^{2}\right)$, and to all orders in velocity for the scattering angles with spins aligned to orbital angular momentum $[59,65]$, whose spin-orbit part is in agreement with Ref. [64]. The latter comparison in the 
spin-orbit case is especially powerful because it verifies the complete coefficient of the spin-orbit operator. In the test body limit, we also reproduce a simplified isotropic gauge version $^{1}$ of the test-body Hamiltonian given in Appendix D of Ref. [75] valid to quadratic order in spin and all order in Newton's constant and velocity. At the amplitude level and with a suitable interpretation of the covariant spin vector, we also recover the spin-1/2 results of Ref. [66]. Although we work with an arbitrary spin, this is expected because, as we argue on general grounds in Sec. II, for the terms linear in the spin of each particle, spin- $1 / 2$ is sufficient as long as no special properties of the Pauli matrices are used $[23,34,62,66]$.

In this paper we use mostly negative metric, and the fourdimensional Levi-Civita symbol is normalized as $\epsilon^{0123}=1$. Unless otherwise specified, the boldface symbols denote spatial three vectors. All four momenta are outgoing. Additional notation can be found in Secs. VE and VIE, where we summarize the results in amplitudes and in EFT.

\section{BASICS FOR SPINNING PARTICLES}

In this section we describe the classical limit of processes involving spinning particles (which we identify with spinning compact astrophysical objects) and review basic facts on spin that we use in later sections. We will see that the spin must be of the same classical order as the orbital angular momentum, and therefore, from a Lagrangian perspective, the classical spinning particles should be represented by higher-spin fields. To describe them we follow Sec. 31 of Ref. [71] and Ref. [72]. This approach has the advantage of giving a simple relation between the classical spin vector and Lorentz generators in the Lagrangian, making it straightforward to construct a robust formalism. We will formulate and use a Lagrangian that captures the gauge-invariant completion of the most general parity-even spin-dependent linear response of a massive particle to a gravitational field. We will show that the trilinear interaction of this Lagrangian is the double copy of similarly general trilinear interactions of higher-spin fields with gluons, thus extending observations of Refs. $[41-43,76]$. Last but not least, we will see that, for suitably chosen couplings, the gravitational stress tensor derived from our Lagrangian reproduces (in the classical limit) that of the Kerr black hole, derived in [58]. Consequently, the scattering amplitude of two Kerr black holes discussed in that reference is also correctly reproduced.

\section{A. The classical limit}

Our goal is to extract the classical potential between two massive spinning bodies from their scattering amplitude. To define the classical limit of an amplitude, we follow the

\footnotetext{
${ }^{1} \mathrm{We}$ thank Justin Vines for providing this form of the Hamiltonian.
}

same path used in Refs. [12,14,15]. Classical physics applies whenever the minimal interparticle separation $|\boldsymbol{b}|$ is much larger than the de Broglie wavelength $\lambda$ of each particle. This macroscopic length scale $|\boldsymbol{b}|$ can be chosen as the impact parameter in a scattering process, or the orbital size of a bound binary system. For incoming particles of momentum $\boldsymbol{p}$, we must then have

$$
|\boldsymbol{b}| \gg \lambda=\frac{1}{|\boldsymbol{p}|},
$$

where we use natural, $\hbar=1$, units. This implies that for any such two-body classical system, the magnitude of orbital angular momentum $\mathrm{L}=|\boldsymbol{L}|$ must be large,

$$
\mathrm{L} \sim|\boldsymbol{p} \times \boldsymbol{b}| \gg 1
$$

The same must hold for all other charges, such as electric charge or spin, that may be carried by classical particles. Indeed, the difference between the classical spin and orbital angular momentum is only in the interpretation of the macroscopic length scale: from internal radius to the impact parameter, and rotating to translational velocities. Thus, in the classical limit, we need the magnitude of the spin, $\mathrm{S}_{i}=\left|\boldsymbol{S}_{i}\right|$, and of the orbital angular momentum, $\mathrm{L}=|\boldsymbol{L}|$, to be commensurate,

$$
\mathrm{S}_{1} \sim \mathrm{S}_{2} \sim \mathrm{L}
$$

The net effect is therefore that classically spinning particles should be described from a field theory point of view by a large-spin limit of higher-spin fields. As we will explain shortly, the details of the calculations imply that at fixed order in Newton's constant and in the number of spin vectors, a finite but sufficiently large spin is sufficient to capture all the relevant contributions.

Since the impact parameter is of order of the inverse momentum transfer in a scattering process, $|\boldsymbol{b}| \sim 1 /|\boldsymbol{q}|$, the classical limit implies the hierarchy

$$
m_{1}, m_{2},|\boldsymbol{p}| \sim \mathrm{L}|\boldsymbol{q}| \sim \mathrm{S}_{i}|\boldsymbol{q}| \gg|\boldsymbol{q}| .
$$

The quantum contributions enter at higher orders in a large $\mathrm{L}$ expansion or, equivalently, higher orders in a small $|\boldsymbol{q}|$ expansion. This gives us the scaling

$$
\mathcal{O}(1 / \mathrm{L}) \sim \mathcal{O}\left(1 / \mathrm{S}_{i}\right) \sim \mathcal{O}(|\boldsymbol{q}|) \sim \mathcal{O}(q) \text { (classical expansion). }
$$

We omit the proper mass scale factors for simplicity. The italic letter denotes four-momentum components. Unless otherwise noted, the classical expansion in this paper includes simultaneously the scaling of spins, orbital angular momentum, and $\boldsymbol{q}$. For example, monomials in 
$\boldsymbol{q} \cdot \boldsymbol{S}_{i}$ or their covariant version $q \cdot S_{i}$ are of $\mathcal{O}(1)$ in the classical limit.

A second expansion parameter is the ratio between spin and orbital angular momentum, which is suppressed by the internal size over impact parameter or orbital radius if we ignore the difference in rotating velocities. Therefore the expansion in spin-induced multipole moments is

$$
\begin{aligned}
\mathcal{O}\left(\mathrm{S}_{i} / \mathrm{L}\right) \sim & \mathcal{O}\left(\mathrm{S}_{i} /|\boldsymbol{b}|\right) \sim \mathcal{O}\left(\mathrm{S}_{i}|\boldsymbol{q}|\right) \sim \mathcal{O}\left(\mathrm{S}_{i} q\right) \\
& (\text { spin expansion }) .
\end{aligned}
$$

For examples, the monomials in $\boldsymbol{q} \cdot \boldsymbol{S}_{i}$ are classically $\mathcal{O}(1)$ but are order by order in spin expansion. Indeed, terms linear in the spin correspond to a dipole moment, those quadratic in spin, $\left(\boldsymbol{q} \cdot \boldsymbol{S}_{i}\right)^{2}$, represent a quadrupole moment, etc. While the multipole moments are not necessarily small when taking velocities into account, the fact that we keep the spin vector arbitrary provides a way to classify interactions between two particles in terms of interactions between their respective multipole moments. ${ }^{2}$

The traditional PN expansion parameter relies on velocity $v \sim|\boldsymbol{p}| / m_{i}$. For bound orbits the virial theorem relates the scale of both $G$ and spin expansion parameters to the velocity. ${ }^{3}$ The virial theorem, however, does not hold for unbound orbits, and therefore the velocity expansion is independent from the others in scattering events. From an amplitudes' perspective it is also more natural to keep a fully relativistic velocity dependence. Thus, we will not expand in velocity except to compare with results from the PN literature.

With this in mind, we have the following structure of classical conservative Hamiltonian expanded in the (number of) spin vectors of each particle,

$$
\begin{aligned}
H= & H^{(0)}\left(r^{2}, p^{2}\right)+h_{i}^{(1)}\left(r^{2}, p^{2}\right) \frac{1}{r^{2}} \boldsymbol{L} \cdot \boldsymbol{S}_{i} \\
& +h_{i j}^{(2,1)}\left(r^{2}, p^{2}\right) \frac{1}{r^{4}} \boldsymbol{r} \cdot \boldsymbol{S}_{i} \boldsymbol{r} \cdot \boldsymbol{S}_{j}+\cdots
\end{aligned}
$$

where we only keep terms up to quadratic order in spins, i.e., up to quadrupole moments. Here $\boldsymbol{r}$ and $\boldsymbol{p}$ are center-ofmass distance and momentum and the indices run over $i$, $j=1,2, r=|\boldsymbol{r}|, \quad p=|\boldsymbol{p}| . H^{(0)}\left(r^{2}, p^{2}\right)$ is the spinless

\footnotetext{
${ }^{2}$ In general, multipole moments are symmetric and traceless combinations of spin vectors. At tree level the trace part leads to contact interactions, which are not of interest to us. At loop-level, however, trace terms no longer drop out and more care is needed to relate symmetric products of spin vectors to multipole moment operators. Since in later sections we will be concerned at most with $\operatorname{spin}_{1}-\operatorname{spin}_{2}$ - or dipole-dipole-interactions, we will not need the complete identification of spin-induced multipole moments.

${ }^{3}$ In PN counting, spins are suppressed relative to the angular momentum by $\mathrm{S}_{i} \sim \mathrm{L} v^{\alpha}$, with $\alpha=1$ to 4 depending on rotating speeds. See Ref. [21] for more details.
}

Hamiltonian with the usual PM expansion in $G / r$. At lowest order, ignoring velocity and spin dependence, the potential is simply the Newtonian one. The $h_{b}^{(a)}$ coefficient of each spininduced moment has the same structure as $H^{(0)}\left(r^{2}, p^{2}\right)$. Using $\mathcal{O}(1 / r) \sim \mathcal{O}(|\boldsymbol{q}|)$ under the Fourier transform, we can see that each spin structure is of the same classical order $\mathcal{O}(1)$ as the spinless potential, but carries a higher order in spins, or is equivalently suppressed by the additional powers of $1 / r$. More details will be discussed in Secs. VI and VII. Such Hamiltonians are a basic input into models—-such as the EOB framework [2]-for building gravitational-wave templates. In this paper, we evaluate the $\mathcal{O}\left(G^{2}\right)$ contributions to the conservative two-body potential to all orders in the velocity and to bilinear order in the two spins.

\section{B. The spin vector and tensor}

We now describe the basic field theory formalism that we use to incorporate spin interactions into an amplitude-based approach. In the post-Newtonian framework, the classical spin-orbit and spin-spin interaction Hamiltonian of spinning particles is well-studied in the literature [23,34,62]. A simplifying aspect is that through $\mathcal{O}\left(G^{2}\right)$, spin- $1 / 2$ and spin-1 fields turn out to be sufficient to recover postNewtonian results obtained via general-relativistic methods [51,54,55]. Not surprisingly, at higher orders in spin, calculations using such low-spin fields are insufficient because the dimension of these representations implies that higher powers of Lorentz generator matrices can be expressed in terms of lower powers. For example, the square of a Pauli matrix describing spin- $1 / 2$ is the identity matrix, which is of course not generally true. Thus, to capture all multispin interactions we need a formalism that describes arbitrarily high spins. Such a formalism would also provide an a priori explanation of the validity of the low-spin observations as well as give the minimal value of the spin that is necessary to capture some given spin-induced multipole moment. ${ }^{4}$ Descriptions of higher-spin particles date back to Fierz and Pauli [79]. Our amplitude-based approach to higher spin is closely related to the world-line approaches of Refs. [52,57]. The formalism makes the connection between Lorentz generators in the amplitudes and final spin vectors relatively transparent. Alternative approaches based on the massivespinor-helicity formalism of Ref. [47] are found in Refs. [37,59,60,62,65,67].

In quantum field theory, massive particles of integer spin $s$ are described by symmetric traceless rank-s tensor fields [80],

\footnotetext{
${ }^{4}$ As known for some time in the particle physics phenomenology literature, spin-1/2 is sufficient to capture the dipole moment and spin-1 the quadrupole. See e.g., Ref. [77] and also Ref. [78] for a counting of form factors of an arbitrary-spin particle.
} 
$\phi_{s}{ }^{a_{1} \cdots a_{i} \cdots a_{j} \cdots a_{s}}=\phi_{s}{ }^{a_{1} \cdots a_{j} \cdots a_{i} \cdots a_{s}}, \quad \eta_{a_{1} a_{2}} \phi_{s}{ }^{a_{1} a_{2} a_{3} \cdots a_{s}}=0$.

Additional transversality constraints are necessary [80] to select the part corresponding to fixed spin $s$. The corresponding physical states are described by polarization tensors that are symmetric traceless and transverse in all indices,

$$
\begin{aligned}
\varepsilon^{a_{1} a_{2} \cdots a_{i} \cdots a_{j} \cdots a_{m}} & =\varepsilon^{a_{1} a_{2} \cdots a_{j} \cdots a_{i} \cdots a_{m}}, \quad \eta_{a_{1} a_{2}} \varepsilon^{a_{1} a_{2} \cdots \cdots a_{m}}=0, \\
p_{a_{1}} \varepsilon^{a_{1} a_{2} \cdots \cdots a_{m}} & =0 .
\end{aligned}
$$

The Hermitian Lorentz generators in this representation are

$$
\begin{aligned}
& \left(M^{a b}\right)_{c(s)} d(s)=2 i s \delta_{\left(c_{1}\right.}^{[a} \eta^{b]\left(d_{1}\right.} \delta_{c_{2}}^{d_{2}} \cdots \delta_{\left.c_{s}\right)}^{\left.d_{s}\right)}, \\
& \left(M^{a b}\right)_{c(s) d(s)}=-\left(M^{a b}\right)_{d(s) c(s)},
\end{aligned}
$$

where the indices $c(s)$ and $d(s)$ stand for the symmetrized sets of vector indices $\left\{c_{1}, \ldots, c_{s}\right\}$ and $\left\{d_{1}, \ldots, d_{s}\right\}$, respectively, and they are raised and lowered with the appropriate symmetric product of the Minkowski metric. The generators $M^{a b}$ satisfy the usual Lorentz algebra,

$$
\begin{aligned}
{\left[M^{a_{1} a_{2}}, M^{a_{3} a_{4}}\right]=} & i\left(\eta^{a_{3} a_{1}} M^{a_{4} a_{2}}+\eta^{a_{2} a_{3}} M^{a_{1} a_{4}}\right. \\
& \left.-\eta^{a_{4} a_{1}} M^{a_{3} a_{2}}-\eta^{a_{2} a_{4}} M^{a_{1} a_{3}}\right) .
\end{aligned}
$$

As we will explain shortly, apart from describing the scattering of massive spin- $s$ fields, our interest is to develop a formalism that avoids use of any of the special properties of fixed-spin representations of the Lorentz group. Thus, it suffices for our purpose to not demand that they be transverse and instead treat the fields (2.8) as unconstrained. It is then convenient to follow Refs. [71,72] and map them to a two-component spinor indices in the usual way,

$$
\phi_{s \alpha_{1} \cdots \alpha_{s}}^{\dot{\beta}_{1} \cdots \dot{\beta}_{s}}=\phi_{s}{ }^{a_{1} \cdots a_{s}}\left(\sigma_{a_{1}}\right)_{\left(\alpha_{1}\right.}\left(\dot{\beta}_{1} \cdots\left(\sigma_{a_{s}}\right)_{\alpha_{s}}{ }^{\left.\dot{\beta}_{s}\right)} .\right.
$$

This parametrization trivializes the tracelessness condition (2.8), which translates into symmetrization in the twocomponent spinor indices of the same handedness. Halfinteger spin can also be described along these lines [71,72], as pairs of such fields with different numbers of left-handed and right-handed indices. While we do not discuss them in any detail (and in the classical limit they should give the same result as the integer-spin fields), we will also describe integer-spin fields as pairs of fields (2.12):

$$
\phi_{s}=\frac{1}{\sqrt{2}}\left(\begin{array}{c}
\xi_{\beta_{1} \cdots \alpha_{u}}^{\alpha_{1} \cdots \alpha_{v}} \\
\dot{\beta}_{1} \cdots \dot{\beta}_{v} \\
\chi_{\dot{\alpha}_{1} \cdots \dot{\alpha}_{v}}^{\beta_{1} \cdots \beta_{v}}
\end{array}\right) .
$$

For integer- and half-integer-spin particles we have

$$
u=v=s, \quad u=s+\frac{1}{2}, \quad v=s-\frac{1}{2},
$$

respectively. ${ }^{5}$ For half-integer spins $\xi$ and $\chi$ are different objects; one may impose a Majorana-type condition which identifies one with the conjugate of the other. In the remaining part of this paper we use only integer spin, since that is sufficient for describing large spin.

When taking the classical limit of quantum-mechanical expectation values, it is necessary to choose states that minimize the standard deviation of observables being considered. ${ }^{6}$ For a spin system in the rest frame, the relevant states are the so-called "spin coherent states" [81]. Their defining property is that

$$
\langle\boldsymbol{n} \mid \boldsymbol{n}\rangle=1, \quad\langle\boldsymbol{n}|\hat{\boldsymbol{S}}| \boldsymbol{n}\rangle=\boldsymbol{S} \equiv|\boldsymbol{S}| \boldsymbol{n}, \quad \frac{\Delta \hat{\boldsymbol{S}}}{|\boldsymbol{S}|} \rightarrow 0,
$$

where $\hat{S}$ is the rest-frame spin operator, related to the rotation generator $M_{j k}$ in the usual way, $\hat{S}^{i}=\frac{1}{2} \epsilon^{i j k} M_{j k}$, and $\boldsymbol{n}$ is the unit vector along the classical spin. The state $|\boldsymbol{n}\rangle$ localizes the spin along the unit vector $\boldsymbol{n}$ as much as it is allowed by quantum mechanics.

We define the covariant spin vector and spin tensor of a particle by boosting their rest-frame three-dimensional counterparts $S^{i}$ and $S^{i j}$, which are related in the standard way

$$
S^{i}=\frac{1}{2} \epsilon^{i j k} S_{j k}
$$

The boost from the particle's rest frame gives

$$
\begin{aligned}
& S(p, \boldsymbol{S})^{\mu}=\left(\frac{\boldsymbol{p} \cdot \boldsymbol{S}}{m}, \boldsymbol{S}+\frac{\boldsymbol{p} \cdot \boldsymbol{S}}{m(E+m)} \boldsymbol{p}\right), \\
& S(p, \boldsymbol{S})^{i 0}=-S(p, \boldsymbol{S})^{0 i}=\frac{1}{m} S^{i l} p_{l}=\epsilon^{i l n} \frac{p_{l}}{m} S_{n}, \\
& S(p, \boldsymbol{S})^{i j}=S^{i j}-2 \frac{p^{[i} S^{j] l} p_{l}}{m(m+E)}=\epsilon^{i j k}\left[\frac{E}{m} S_{k}-\frac{\boldsymbol{p} \cdot \boldsymbol{S}}{m+E} \frac{p_{k}}{m}\right],
\end{aligned}
$$

where Roman letters from the middle of the alphabet indicate spatial indices. We raise and lower the indices of the three-dimensional (3D) rest-frame spin vector with the Euclidean 3D metric, so $S^{k}=S_{k}$ (which should not be confused with the spatial part of $S^{\mu}$ ). These expressions can be summarized in a covariant format:

\footnotetext{
${ }^{5}$ One may have more general representations, in which $u$ and $v$ differ by some finite amount, $u=s_{L}, v=s_{R}$, and $s=s_{L}+s_{R}$.

${ }^{6}$ For example, for the harmonic oscillator, classical physics is recovered if one chooses it to be in a coherent state.
} 


$$
\begin{aligned}
S^{\alpha \beta}(p) & =-\frac{1}{m} \epsilon^{\alpha \beta \gamma \delta} p_{\gamma} S_{\delta}(p), \\
S^{\alpha}(p) & =-\frac{1}{2 m} \epsilon^{\alpha \beta \gamma \delta} p_{\beta} S_{\gamma \delta}(p),
\end{aligned}
$$

where the four-dimensional Levi-Civita symbol is normalized as $\epsilon^{0123}=1$. We will later denote the covariant spin vector of particle $a$ as $S_{a} \equiv S\left(p_{a}, S_{a}\right)$. Our definition of the covariant spin vector implies that it obeys the so-called covariant spin supplementary condition,

$$
p_{\mu} S(p, S)^{\mu}=0 .
$$

Boosting the relations (2.15) to an arbitrary frame will not change the scalar product of coherent states and will yield the covariant spin vector on the right-hand side of the second equation. Boosting the ket and the bra states to momenta differing by some momentum transfer $q$ is less trivial. It is lengthy but straightforward to show that [see also Appendix E of Ref. [60] for some details on the derivation of $\left.\varepsilon\left(\boldsymbol{s}, p_{1}\right) \cdot \varepsilon\left(\boldsymbol{s}, p_{2}\right)\right]$

$$
\begin{aligned}
& \varepsilon\left(\boldsymbol{s}, p_{1}\right) \cdot \varepsilon\left(\boldsymbol{s}, p_{2}\right) \\
& \quad=\left(1-i \frac{\epsilon_{r s k} p_{1}^{r} p_{2}^{s} S^{k}}{m\left(m+E\left(\boldsymbol{p}_{1}\right)\right)}+\mathcal{O}\left(\mathrm{S}^{2} \boldsymbol{q}^{2}\right)\right)+\mathcal{O}(q), \\
& \varepsilon\left(\boldsymbol{s}, p_{1}\right) M^{a b} \varepsilon\left(\boldsymbol{s}, p_{2}\right) \\
& \quad=S\left(p_{1}, \boldsymbol{S}\right)^{a b} \varepsilon\left(\boldsymbol{s}, p_{1}\right) \cdot \varepsilon\left(\boldsymbol{s}, p_{2}\right)+\mathcal{O}\left(q^{0}\right), \\
& \varepsilon\left(\boldsymbol{s}, p_{1}\right) \frac{1}{2}\left\{M^{a b}, M^{c d}\right\} \varepsilon\left(\boldsymbol{s}, p_{2}\right) \\
& \quad=S\left(p_{1}, \boldsymbol{S}\right)^{a b} S\left(p_{1}, \boldsymbol{S}\right)^{c d} \varepsilon\left(\boldsymbol{s}, p_{1}\right) \cdot \varepsilon\left(\boldsymbol{s}, p_{2}\right)+\mathcal{O}\left(q^{-1}\right),
\end{aligned}
$$

where $\varepsilon\left(s, p_{1}\right)$ and $\varepsilon\left(\boldsymbol{s}, p_{2}\right)$ are the incoming and outgoing polarization tensors of a particle in a convention where both momenta are taken to be outgoing, so that the momentum transfer is $q=-p_{1}-p_{2}$. We denote the spin label by $s$ to emphasize that, in general, it can be a quantum property of the particle and to distinguish it from the rest-frame classical spin vector $S$. The rest-frame spin is assumed to be large in the classical limit, with $\boldsymbol{q} \cdot \boldsymbol{S} / m \sim \mathcal{O}(1)$ as discussed in Sec. II A, and the number of left-handed and right-handed indices in Eq. (2.13), $u$ and $v$, are commensurate, i.e., $u-v \ll u, v$. The terms in the parentheses of the first line are classical, and we only exhibit them to linear order in spin. The rest of $\mathcal{O}\left(q^{\alpha}\right)$ denote classical expansion, where we show only the leading term. Recall that classical expansion count both $\boldsymbol{q}$ and spins. The momentum of the spin tensor can be chosen to be any combination of $p_{1}$ and $p_{2}$ since all such combinations differ by terms proportional to the momentum transfer $q$. In tree amplitudes the momentum dependence of vertices makes all these contributions subleading in the classical limit. We will revisit the subleading terms in the second of Eq. (2.20) in our discussion of the effective field theory and the comparison of its amplitudes with those of the higher-spin Lagrangian we discuss next. As we will see, they do not affect observables. Another subtlety is that, as indicated in the final formula in Eq. (2.20), only the symmetric product of Lorentz generators is interpreted directly as products of spin tensors. As will be discussed in Sec. III, this is sufficient for obtaining the classical limit of a product of two Lorentz generators after accounting for the standard commutation relation (2.11).

\section{Higher-spin Lagrangians}

Theories of massive higher-spin fields have a long history. A free action was constructed in Ref. [80]. Spin- $s$ fields are described by rank- $s$ symmetric tensors. Fields transforming in the $(s+1, s+1)$ representation of the $S O(3,1) \simeq S U(2) \times S U(2)$ Lorentz group $^{7}$ contain many representations of the rotation group. To eliminate all but the spin-s representation [i.e., the $(2 s+1)$-dimensional representation of the $S O(3)$ rotation group], the tensor field is usually constrained to be transverse. Implementing this in a Lorentz-invariant Lagrangian can be done [80] with the aid of $s$ rank- $k$ auxiliary fields, with $k=0, \ldots, s-1$. Preservation of tree-level unitarity when coupling this free action with gravity turns out to require introduction of dimension-four terms involving both the higher-spin fields and the Riemann curvature tensor $[82,83]$. Aspects of an interacting Lagrangian constructed along these lines for all spin-induced multipole moments were discussed in Ref. [62].

While we use a Lagrangian to organize the interactions of higher-spin fields with gravity, we take a different approach than earlier ones, which is tailored to our needs for constructing classical limits of amplitudes with explicit dependence on the spin vector. It may be interpreted as a relativistic effective theory that captures all spin-induced multipole moments and thus all linear responses of spinning objects to gravity. This approach provides a minimal completion of any desired three-particle interactions which is invariant under the nonlinear diffeomorphism transformations and offers a convenient way to align our derivations with earlier ones. Here we will use this approach to understand aspects of low-point interactions of higher-spin fields and gravitons in the classical limit and derive the effective interaction potential of two higher-spin fields due to the exchange of gravitons through $\mathcal{O}\left(G^{2}\right)$.

For our purpose it is not important that the matter fields transform in an irreducible representation of the rotation group. It is, however, important that all irreducible components be treated uniformly. To this end we take our fields to be traceless rank- $s$ tensors in their spinor

\footnotetext{
${ }^{7}$ We denote representations of the Lorentz group by $\left(d_{L}, d_{R}\right)$, where the two entries are the dimensions of the two $S U(2)$ representations.
} 
formulation (2.12) and not require that they be transverse. The validity of this approach can be verified a posteriori through the independence of the result on the number of components of the tensor field. One may intuitively expect that this will be the case as the number of components of matter fields can arise only from loops containing them and such graphs do not contribute in the classical limit. As we will see, this framework provides a minimal value for the spin needed to capture the complete spin dependence of an $L$-loop four-point amplitude in the classical limit. Such lower bonds are similar in spirit with the observation $[23,34,62]$ that calculations at fixed and low spins can be used to reproduce the part of the spin-dependent Hamiltonian that is available in the literature and was originally derived though general-relativistic techniques.

We describe the gravitational field in the vielbein rather than the metric formulation because it exposes the tangentspace Lorentz generators, making it easier to identify the (classical) spin vector. Since we are not interested in matter contact interactions of higher-spin fields (because they do not contribute to the long-range potential), we will focus on a single higher-spin field, $\phi_{s}$.

Our higher-spin Lagrangian has two parts:

$$
\mathcal{L}=\mathcal{L}_{\text {min }}+\mathcal{L}_{\text {nonmin }}, \quad S=\int d^{4} x \sqrt{-g} \mathcal{L} .
$$

The minimal Lagrangian, i.e., the Lagrangian with the minimal number of derivatives, including the terms needed to preserve tree-level unitarity [82-84], is ${ }^{8}$

$$
\begin{aligned}
\mathcal{L}_{\min }= & -R(e, \omega)+\frac{1}{2} g^{\mu \nu} \nabla(\omega)_{\mu} \phi_{s} \nabla(\omega)_{\nu} \phi_{s}-\frac{1}{2} m^{2} \phi_{s} \phi_{s} \\
& +\frac{\mathrm{H}}{8} R(e, \omega)_{e f g h} \phi_{s} M^{e f} M^{g h} \phi_{s}+\cdots,
\end{aligned}
$$

where $\mathrm{H}$ is an adjustable parameter, we take the higher-spin field $\phi_{s}$ to be real, and the ellipses stand for terms that vanish on shell. The $M^{a b}$ are the Hermitian Lorentz generators in the $(s+1, s+1)$ representation (2.12) and (2.13). The covariant derivative is

$$
\nabla(\omega)_{\mu} \phi_{s} \equiv \partial_{\mu} \phi_{s}+\frac{i}{2} \omega_{\mu e f} M^{e f} \phi_{s}
$$

where $\omega$ is the spin connection. To shorten the expression we do not display the many tangent-space indices of $\phi_{s}$. They are understood as contracted via matrix multiplication. The spinor notation we use for the higher-spin field emphasizes that they are assumed to carry only tangentspace indices; similarly, the curvature tensor and the Lorentz generators also carry tangent-space indices. We

\footnotetext{
${ }^{8}$ The sign of the $\mathrm{H}$ term follows from the one in [82-84] by changing the signature of the metric to mostly minus and converting to Hermitian Lorentz generators.
}

postpone describing nonminimal higher-spin Lagrangians to the next section.

The last term displayed in Eq. (2.22) is a gravitational quadrupole interaction; its coefficient may be set to $\mathrm{H}=1$ by requiring that amplitudes have an improved high-energy behavior, delaying violations of partial-wave unitarity $[83,84]$. String theory predicts a different value for this coefficient [84]. This may be interpreted as being due to the other higher-spin fields of string theory further contributing to the unitarity constraint. Here we keep $\mathrm{H}$ as a free parameter. This term does not affect any interaction linear in the particle's spin, but it is important at higher order in spin and, as we will see in Sec. III B, plays an important role in giving a field-theory description of the stress tensor of the Kerr black hole [58]. The value of $\mathrm{H}$ found by matching to a Kerr black hole reproduces the one required by improved partial-wave unitarity.

At tree level there is no physical difference between the scattering of higher-spin fields described by the Lagrangian (2.22) and by one that enforces transversality of the higherspin polarization vectors. This is because four-point treelevel scattering amplitudes of higher-spin fields contain no Feynman graphs with propagators for these fields.

Let us now examine the relation between calculations carried out with low-spin fields and with arbitrary-spin fields, beyond tree level and for the case where each vertex contains no more than one Lorentz generator. The Lagrangians for massive vectors and massive spin-2 fields without curvature couplings are both of the form of Eq. (2.22). Theories of such low-spin fields will yield the same amplitudes as the Lagrangian (2.22) as long as special relations obeyed by symmetric products of generators of four-dimensional Lorentz group in representations $(2,2)$ and $(3,3)$ are not used. These relations stem from the fact that, for a spin-s representation of $S U(2)$, with generators $J_{s}$,

$$
\left(\Xi \cdot J_{s}\right)^{k \geq 2 s+1}=\sum_{n=1}^{2 s} a_{n}(\Xi, k, s)\left(\Xi \cdot J_{s}\right)^{n}
$$

for some coefficients $a_{n}(\Xi, k, s)$. Here $\Xi$ is an arbitrary three-component vector; differentiating Eq. (2.24) $k$ times with respect to $\Xi$ yields the decomposition of a symmetric product of $k$ generators of $S O(3)$ into a linear combinations of symmetric products of at most $s$ generators. If each matter-graviton vertex of a Feynman diagram of an $L$-loop four-point matter amplitude contains at most one Lorentz generator, it is easy to see that each matter line of this diagram contains a (symmetric) product of at most $L+1$ generators, multiplied from the left and the right with polarization tensors. For (2.24) not to operate if the fields are in a chiral representation of the Lorentz group [i.e., they transform under only one of the two $S U(2)$ factors], it is therefore necessary that they be in a representation of dimension $\operatorname{dim} \geq L+2$. 
This counting suggests that one-loop calculations carried out with fields with the Lorentz representations $(3,1)$ or/and $(1,3)$ should be sufficient within our formalism because they yield products of at most two Lorentz generators (in e.g., box or triangle graphs) for a matter line. At two loops, where we may get products of three Lorentz generators, Lorentz representations of the type $(4,1)$ or/and $(1,4)$ are needed. We interpret these bounds as being sufficient to capture the complete spin dependence within our formalism. Similarly, on a case-by-case basis it may be possible to evade them and use e.g., spin-1 fields in our formalism and capture the complete spin dependence at one-loop. One may reach this conclusion by e.g., constructing a relation analogous to $(2.24)$ for the $S O(3,1)$ generators in the $(2,2)$ representation and demanding that the decomposition be manifestly covariant.

We stress that the counting above refers specifically to our formalism and does not necessarily apply to actions that e.g., use properties of special representations of $S O(3,1)$. The actual bound might even be lowered after the classical limit is applied. In general, perhaps the most straightforward approach to using low values of spin is to use a formulation of the low-spin Lagrangians that does not implicitly employ relations between Lorentz generators that use their four-dimensional nature. Moreover, such relations should not be used at any step in the calculation of amplitudes.

Trilinear couplings containing more than one Lorentz generator, such as the H-dependent term in (2.22) and the higher-derivative terms discussed below change the counting argument above and suggest a need for larger representations at lower-loop orders.

\section{Expansion of the minimal Lagrangian}

The spin connection $\omega$ is an auxiliary field, which can be eliminated via its equation of motion, as usually done in supergravity theories. This expresses $\omega$ in terms of the vielbein and matter fields. Once replaced in the original Lagrangian, the matter-field dependence yields only matter contact terms and is thus irrelevant for long-range interactions of matter fields. The remainder matter-independent solution of the $\omega$ equation of motion is equivalent to the solution to the vielbein postulate, $\nabla_{\mu}(\omega) e_{\nu}{ }^{a}=0$. We will denote it by $\omega(e)$.

Following standard methods we define the graviton field as the fluctuation of the metric around the Minkowski background. Local Lorentz symmetry can be used to choose the fluctuations of the vielbein to be symmetric, $h_{\mu a}=h_{a \mu}$. We take

$$
\begin{gathered}
g_{\mu \nu}=\eta_{\mu \nu}+h_{\mu \nu}, \quad e_{\mu}{ }^{a}=\delta_{\mu}^{a}+\frac{1}{2} h_{\mu}{ }^{a}-\frac{1}{8} h_{\mu \rho} h^{a \rho}+\mathcal{O}\left(h^{3}\right), \\
\omega(e)_{\mu c b}=-\partial_{[c} h_{b] \mu}-\frac{1}{4} h^{\rho}{ }_{[c} \partial_{\mu} h_{b] \rho}+\frac{1}{2} h^{\rho}{ }_{[c} \partial_{\rho} h_{b] \mu}-\frac{1}{2} h^{\rho}{ }_{[c} \partial_{b]} h_{\mu \rho}+\mathcal{O}\left(h^{3}\right),
\end{gathered}
$$

where the antisymmetrization includes division by the number of terms and we take $\eta_{\mu \nu}$ to be in the mostly minus convention. One may make different choices for the metric fluctuations to e.g., make the expansion of the vielbein simpler; while this has no effect on scattering amplitudes, it makes gravitational vertices depart from their standard form. With the choice above, the expansion of the Riemann tensor to second order in fluctuations is

$$
\begin{aligned}
R(e, \omega(e))_{e f g h}= & -2 \partial_{[e \mid} \partial_{[g} h_{h] \mid f]}+\left(h^{\mu}{ }_{[e} \delta_{f]}^{\nu}+\delta_{[e}^{\mu} h_{f]}^{\nu}\right) \partial_{\mu} \partial_{[g} h_{h] \nu}-\frac{1}{2} \partial_{[e \mid} h_{[g} \partial_{\mid f]} h_{h] \rho}+\partial_{[e \mid} h_{[g}^{\rho} \partial_{\rho} h_{h] \mid f]} \\
& -\partial_{[e \mid} h^{\rho}{ }_{[g} \partial_{h]} h_{\mid f] \rho}+2 \partial_{[g} h_{c][e \mid} \partial_{[d} h_{h] \mid f]} \eta^{c d}+\mathcal{O}\left(h^{3}\right) .
\end{aligned}
$$

Following the usual procedure we can extract Feynman vertices. Consider the three-point vertex in Fig. 2. The contribution from the three vertex from the minimal Lagrangian (2.22) is then

$$
\begin{aligned}
& -i V_{\min a(s)}^{\mu \nu} b(s)\left(q, p_{1}, p_{2}\right)=p_{1}^{(\mu} p_{2}^{\nu)} \delta_{a(s)}^{b(s)}-\frac{1}{2} \eta^{\mu \nu}\left(p_{1} \cdot p_{2}+m^{2}\right) \delta_{a(s)}^{b(s)}-\frac{i}{2} q_{\rho}\left(p_{2}-p_{1}\right)^{(\mu}\left(M^{\nu) \rho}\right)_{a(s)}^{b(s)} \\
& +\frac{\mathrm{H}}{2} q_{\rho} q_{\sigma}\left(M^{\rho(\mu \mid} M^{\sigma \mid \nu)}\right)_{a(s)} b^{b(s)},
\end{aligned}
$$

where the legs carrying momenta $p_{1}$ and $p_{2}$ are spin-s fields with sets of tangent-space Lorentz indices $b(s)$ and $a(s)$, respectively, and the symmetrization of the two graviton indices has unit strength (i.e., it includes division by the number of terms). A useful property of the vertex, following from diffeomorphism invariance of the action, is the on-shell analog of stress tensor conservation. That is, when its external legs are placed on shell, the three-point vertex is transverse with respect to the graviton momentum, 


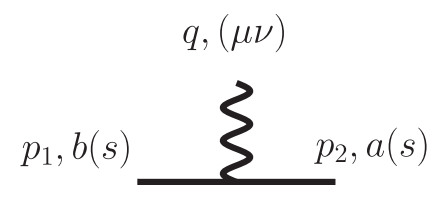

FIG. 2. The three vertex labels. All momenta are outgoing.

$$
\begin{aligned}
& q_{\mu} V_{\min a(s)}^{\mu \nu} b(s)\left(q, p_{1}, p_{2}\right)=0, \\
& q_{\nu} V_{\min a(s)}^{\mu \nu} b(s)\left(q, p_{1}, p_{2}\right)=0 .
\end{aligned}
$$

This does not require any special properties, such as transversality, for the higher-spin polarization tensors. This is consistent with our setup, in which higher-spin fields are not required to be transverse. As for low-spin particles, this property guarantees the gauge-choice independence of the tree-level four-point matter amplitude.

\section{NONMINIMAL INTERACTIONS}

The general form of the stress tensor of an arbitrary-spin particle in a parity-invariant theory is described in Ref. [72] in terms of four independent form factors. When used as vertices in a scattering amplitude, two of them contribute only contact terms. In this section we describe a nonminimal part $\mathcal{L}_{\text {non-min }}$, corresponding to the remaining two form factors of the Lagrangian (2.22).

\section{A. Nonminimal higher-spin Lagrangians and cubic vertices}

To construct manifestly covariant spin-dependent Lagrangian interactions it is convenient to define an offshell manifestly covariant analog of the Pauli-Lubanski vector:

$$
\mathbb{S}^{a} \equiv \frac{-i}{2 m} \epsilon^{a b c d} M_{c d} \nabla(\omega)_{b}
$$

It carries an explicit tangent-space vector index and two implicit labels for the spin representation which we will choose to be $(s+1, s+1)$. Then, all the terms linear in the graviton and bilinear in higher-spin fields are

$$
\begin{aligned}
\mathcal{L}_{\text {non-min }}= & \sum_{n=1}^{\infty} \frac{(-1)^{n}}{(2 n) !} \frac{C_{E S^{2 n}}}{m^{2 n}} \nabla(\omega)_{f_{2 n}} \ldots \nabla(\omega)_{f_{3}} R_{f_{1} a f_{2} b} \nabla(\omega)^{a} \phi_{s} \mathbb{S}^{\left(f_{1}\right.} \ldots \mathbb{S}^{\left.f_{2 n}\right)} \nabla(\omega)^{b} \phi_{s} \\
& \left.-\sum_{n=1}^{\infty} \frac{(-1)^{n}}{(2 n+1) !} \frac{C_{B S^{2 n}}}{m^{2 n+1}} \nabla(\omega)_{f_{2 n+1}} \cdots \nabla(\omega)_{f_{3}} \frac{1}{2} \epsilon_{a b\left(c \mid f_{1}\right.} R^{a b} \mid d\right) f_{2} \nabla(\omega)^{c} \phi_{S} \mathbb{S}^{\left(f_{1} \ldots \mathbb{S}^{\left.f_{2 n+1}\right)} \nabla(\omega)^{d} \phi_{s},\right.}
\end{aligned}
$$

where, as in Eq. (2.22), the indices on $\phi_{s}$ are implicit. The operators included here are in one-to-one correspondence to the nonminimal couplings in the world-line spinning-particle action of Ref. [57]. As in the minimal Lagrangian, here all indices are flat, and we assume that the fields are real.

We note that, through cubic order in fields, the matrix elements of the quadrupole term in (2.22) are indistinguishable from the matrix elements of the $n=1$ term on the first line of Eq. (3.2). Thus, when combining the minimal and nonminimal Lagrangians and their contributions to vertices, we will drop the $\mathrm{H}$ term in favor of $C_{E S^{2}}$.

One can include further terms, with two or more Riemann tensors. At $\mathcal{O}(G)$ in a scattering process, they necessarily imply emission of gravitational radiation. At higher orders in Newton's constant they contribute also to the conservative part of the two-particle spin-dependent Hamiltonian. Some of them can be identified as bilinears in the single-graviton operators included in Eq. (3.2). We will not attempt to classify here all such operators to all orders in spin.

To find the nonminimal vertices we expand (3.2) around Minkowski space. Following the same reasoning as in Sec. II D, we may take the spin connection to be given by the solution to the vielbein postulate. This implies that the expansion of the Riemann tensor is given by Eq. (2.26). Since all nonminimal terms contain a Riemann tensor, none of the other vielbein or spin connections in Eq. (3.2) contribute to the three-point vertex. It is convenient to consider separately the contribution of the terms depending on the Riemann tensor and its dual:

$$
-i V_{3 \text { non- } \min }^{\mu \nu}=-i V_{3 \text { non- } \min , E}^{\mu \nu}-i V_{3 \text { non- } \min , B}^{\mu \nu} .
$$

Each of them is given by

$$
\begin{aligned}
& -i V_{3 \text { non- } \min , E}^{\mu \nu}=p_{1}^{(\mu} p_{2}^{\nu)} \operatorname{Sym}\left[\left(q \cdot \mathbb{S}_{0}\left(p_{1}\right)\right),\left(q \cdot \mathbb{S}_{0}\left(p_{1}\right)\right), \hat{E}\left(q \cdot \mathbb{S}_{0}\left(p_{1}\right)\right)\right]+\mathcal{O}\left(q, p_{i}^{2}-m^{2}\right), \\
& -i V_{3 \text { non- } \min , B}^{\mu \nu}=-\frac{i}{2} m^{2} q_{\rho}\left(p_{2}-p_{1}\right)^{(\mu} \operatorname{Sym}\left[M^{\nu) \rho}, q \cdot \mathbb{S}_{0}\left(p_{1}\right), \hat{B}\left(q \cdot \mathbb{S}_{0}\left(p_{1}\right)\right)\right]+\mathcal{O}\left(q, p_{i}^{2}-m^{2}, p_{i}^{\mu} M_{\mu \nu}\right),
\end{aligned}
$$


where $\mathbb{S}_{0}(p)^{\mu}$ is the Fourier transform of the linearization of the operator $\mathbb{S}^{\mu}$ in Eq. (3.1) (i.e., the part that is independent of the metric fluctuations),

$$
\mathbb{S}_{0}(p)^{\mu} \equiv \frac{1}{2 m} \epsilon^{\mu \nu \rho \sigma} p_{\nu} M_{\rho \sigma} .
$$

In the second Eq. (3.4) we neglected terms containing $p_{i}^{\mu} M_{\mu \nu}$ because such terms vanish in the classical limit up to contributions subleading in $q$. The operators $\hat{E}(X)$ and $\hat{B}(X)$ are defined as

$$
\begin{aligned}
& \hat{E}(X)=\sum_{n=1}^{\infty} \frac{1}{(2 n) !} \frac{C_{E S^{2 n}} m^{2 n}}{{ }^{2 n-2}}, \\
& \hat{B}(X)=\sum_{n=1}^{\infty} \frac{1}{(2 n+1) !} \frac{C_{B S^{2 n+1}} m^{2 n+2}}{{ }^{2 n-1},}
\end{aligned}
$$

and the operator $\operatorname{Sym}[\cdots]$ symmetrizes with unit strength in all of its arguments. Last, if an argument is the $n$th power of an operator, then it is interpreted as $n$ distinct entries.

\section{B. The higher-spin and the Kerr black hole stress tensor}

To construct the on-shell stress tensor we contract the three-point vertex with polarization tensors for the higherspin fields, use the mass-shell conditions and transversality of the graviton polarization tensor,

$q^{2}=0, \quad q^{\mu} \varepsilon(q)_{\mu \nu}=q^{\nu} \varepsilon(q)_{\mu \nu}=0, \quad p_{1}^{2}=p_{2}^{2}=m^{2}$,

and evaluate

$$
T^{\mu \nu}=\frac{i}{m} \frac{\varepsilon\left(\boldsymbol{s}, p_{2}\right)\left(V_{3, \min }^{\mu \nu}+V_{3, \text { non-min }}^{\mu \nu}\right) \varepsilon\left(\boldsymbol{s}, p_{1}\right)}{\varepsilon\left(\boldsymbol{s}, p_{2}\right) \cdot \varepsilon\left(\boldsymbol{s}, p_{1}\right)} .
$$

Following our original setup, we do not assume that the higher-spin polarization tensors are transverse. The division by $\varepsilon\left(\boldsymbol{s}, p_{2}\right) \cdot \varepsilon\left(\boldsymbol{s}, p_{1}\right)$ can be understood as a choice of position-space coordinate conjugate to the graviton momentum. With this normalization this coordinate is covariant and thus includes a certain shift proportional to the rest-frame spin [72].

To see this it is useful to recall that, as it was understood long ago by Foldy and Wouthuysen [85] in the context of the free Dirac theory, the operator $\boldsymbol{x}_{\text {cov }}$ whose expectation value is the position of a particle is, in fact, a particular combination of the canonical position operator $\boldsymbol{x}$ and the spin operator. For a particle with momentum $p$ and restframe spin $S$ it is

$$
\boldsymbol{x}_{\mathrm{cov}}=\boldsymbol{x}-\frac{\boldsymbol{p} \times \boldsymbol{S}}{m(E(\boldsymbol{p})+m)} .
$$

A similar relation was shown in Ref. [58] to be a consequence of switching between the covariant and canonical spin supplementary conditions. Using the allorders-in-spin generalization of Eqs. (2.20),

$\varepsilon\left(\boldsymbol{s}, p_{1}\right) \cdot \varepsilon\left(\boldsymbol{s}, p_{2}\right)=\exp \left[-i \frac{\epsilon_{r s k} p_{1}^{r} p_{2}^{s} S^{k}}{m(E+m)}\right]+\mathcal{O}(q)$,

which may be proven directly, by writing the polarization tensors as boosts of rest-frame coherent states, as in Sec. II, it is straightforward to see that

$$
\begin{aligned}
& \int d^{2} \boldsymbol{q} e^{-i \boldsymbol{r} \cdot \boldsymbol{q}} \varepsilon\left(\boldsymbol{s}, p_{2}\right)\left(V_{3, \text { min }}^{\mu \nu}+V_{3, \text { non-min }}^{\mu \nu}\right) \varepsilon\left(\boldsymbol{s}, p_{1}\right) \\
&=\int d^{2} \boldsymbol{q} e^{-i \boldsymbol{r}_{\mathrm{cov}} \cdot \boldsymbol{q}} \frac{\varepsilon\left(\boldsymbol{s}, p_{2}\right)\left(V_{3, \text { min }}^{\mu \nu}+V_{3, \text { non- } \min }^{\mu \nu}\right) \varepsilon\left(\boldsymbol{s}, p_{1}\right)}{\varepsilon\left(\boldsymbol{s}, p_{2}\right) \cdot \varepsilon\left(\boldsymbol{s}, p_{1}\right)} .
\end{aligned}
$$

This choice facilitates comparisons with Ref. [58], which uses the covariant coordinate and covariant spin tensor in the derivation of the Kerr black hole stress tensor. To express our results in terms of the rest-frame spin, it is necessary to restore the spin dependence contained in the product of polarization tensors; we will do so in later sections.

The classical limit of Eq. (3.8) can be taken by using a generalization of Eq. (2.20) to the symmetric product of an arbitrary number of Lorentz generators. By boosting from the rest frame, where such products can be computed using the properties of the coherent states and the explicit forms of Lorentz generators, it is not difficult to find that

$$
\begin{aligned}
\varepsilon\left(\boldsymbol{s}, p_{2}\right) & \operatorname{Sym}\left[M^{\mu_{1} \nu_{1}}, \ldots, M^{\mu_{n} \nu_{n}}\right] \varepsilon\left(\boldsymbol{s}, p_{1}\right) \\
= & S\left(p_{1}, \boldsymbol{S}\right)^{\mu_{1} \nu_{1}} \cdots S\left(p_{1}, \boldsymbol{S}\right)^{\mu_{n} \nu_{n}} \varepsilon\left(\boldsymbol{s}, p_{2}\right) \cdot \varepsilon\left(\boldsymbol{s}, p_{1}\right) \\
& +\mathcal{O}\left(q^{-(n-1)}\right) .
\end{aligned}
$$

This relation can be used to evaluate the expectation value of a generic product of Lorentz generators. Indeed, using the Lorentz algebra one can rewrite an arbitrary monomial in Lorentz generators as a sum of completely symmetric products or generators, with coefficients given by the structure constants of the algebra. For example,

$$
\begin{aligned}
M^{\mu_{1} \nu_{1}} M^{\mu_{2} \nu_{2}}= & \frac{1}{2}\left\{M^{\mu_{1} \nu_{1}}, M^{\mu_{2} \nu_{2}}\right\}+\frac{1}{2}\left[M^{\mu_{1} \nu_{1}}, M^{\mu_{2} \nu_{2}}\right] \\
= & \frac{1}{2}\left\{M^{\mu_{1} \nu_{1}}, M^{\mu_{2} \nu_{2}}\right\}+\frac{i}{2}\left(\eta^{\mu_{3} \mu_{1}} M^{\mu_{4} \mu_{2}}\right. \\
& \left.+\eta^{\mu_{2} \mu_{3}} M^{\mu_{1} \mu_{4}}-\eta^{\mu_{4} \mu_{1}} M^{\mu_{3} \mu_{2}}-\eta^{\mu_{2} \mu_{4}} M^{\mu_{1} \mu_{3}}\right) .
\end{aligned}
$$

Then, the expectation value of each factor can be evaluated using Eq. (3.12). Each time Lorentz algebra is used, the number of generators decreases by one; the expectation 
value of the resulting monomials is subleading compared to that of the symmetric product of the original number of generators. Such subleading terms will be crucial at oneloop in Sec. V to obtain the correct classical terms. By the same reasoning, this will be true at higher loops as well.

At tree level, however, the maximal number of generators already gives a classical contribution, so all comparatively subleading terms can be ignored. Upon using Eq. (3.12), all Lorentz generators $M^{\mu \nu}$ become spin tensors. Moreover, using the contraction of Eq. (2.18) with the momentum transfer $q$,

$\frac{1}{2} \epsilon_{\mu \nu \rho \sigma} q^{\mu} p_{1}^{\nu} S\left(p_{1}\right)^{\rho \sigma}=-m q_{\mu} S\left(p_{1}\right)^{\mu} \equiv-m q \cdot S\left(p_{1}\right)$,

ignoring terms subleading in the small- $q$ expansion and defining $C_{E S^{0}}=1$ and $C_{B S^{0}}=1$, the stress tensor becomes

$$
\begin{aligned}
T^{\mu \nu}\left(p_{1}, q\right)= & \frac{p_{1}^{\mu} p_{1}^{\nu}}{m} \sum_{n=0}^{\infty} \frac{C_{E S^{2 n}}}{(2 n) !}\left(\frac{q \cdot S\left(p_{1}\right)}{m}\right)^{2 n} \\
& -\frac{i}{m} q_{\rho} p_{1}^{(\mu} S\left(p_{1}\right)^{\nu) \rho} \sum_{n=1}^{\infty} \frac{C_{B S^{2 n+1}}}{(2 n+1) !}\left(\frac{q \cdot S\left(p_{1}\right)}{m}\right)^{2 n} .
\end{aligned}
$$

As expected, it has a form consistent with the general stress tensor that contributes to long-range interactions [72].

Equation (3.14) also implies that, as stated previously, the coefficient $C_{E S^{2}}$ is equivalent to the quadrupole $\mathrm{H}$ term in Eq. (2.22). Indeed, using the relation between the covariant spin vector and tensor it is not difficult to show that

$$
\begin{aligned}
S\left(p_{1}\right)^{\mu \rho} S\left(p_{1}\right)^{\nu \sigma} q_{\rho} q_{\sigma} & =-\frac{1}{m^{2}} p_{1}^{\mu} p_{1}^{\nu}\left(q \cdot S\left(p_{1}\right)\right)^{2}+\ldots \\
& =+\frac{1}{m^{2}} p_{1}^{(\mu} p_{2}^{\nu)}\left(q \cdot S\left(p_{1}\right)\right)^{2}+\cdots,
\end{aligned}
$$

where the ellipses stand for terms that vanish when the free indices are contracted with an on-shell graviton polarization tensor. Thus, as noted earlier, we are justified to ignore the quadrupole term in Eq. (2.22) when the nonminimal interaction Lagrangian (3.2) is included. Comparing Eq. (2.27) with the $n=1$ term in the first Eq. (3.4) in the classical limit and using (3.16), it is easy to see that the coefficient $\mathrm{H}$ is related to $C_{E S^{2}}$ as

$$
\mathrm{H}=C_{E S^{2}}
$$

Thus, the value of $\mathrm{H}$ for the Kerr black hole can be found by comparing Eq. (3.15) to the stress tensor of the Kerr black hole constructed in Ref. [58].
To carry out this comparison we first organize the result of Ref. [58] in our notation. It is found there by casting the linearized Kerr metric in the form of an operator acting on a free-particle Green's function:

$$
\begin{aligned}
h_{\rho \sigma} & =4 G \mathcal{P}_{\rho \sigma \mu \nu}^{\text {de Donder }} \hat{T}^{\mu \nu} \frac{1}{r}, \\
\mathcal{P}_{\rho \sigma \mu \nu}^{\text {de Donder }} & =\frac{1}{2} \eta_{\mu \alpha} \eta_{\nu \beta}+\frac{1}{2} \eta_{\nu \alpha} \eta_{\mu \beta}-\frac{1}{D-2} \eta_{\mu \nu} \eta_{\alpha \beta} .
\end{aligned}
$$

Here $\mathcal{P}_{\rho \sigma \mu \nu}^{\mathrm{deD} D o n d e r}$ is the tensor structure of the graviton propagator in de Donder gauge and $r$ is the flat-space four-dimensional coordinate distance, $r^{2}=\eta_{\mu \nu} x^{\mu} x^{\nu}$. The stress-tensor operator $\hat{T}^{\mu \nu}$ is given by [cf. Eq. (32a) of Ref. [58] ]

$$
\hat{T}^{\mu \nu}=m \exp (a * \partial)_{\rho}^{(\mu} u^{\nu)} u^{\rho},
$$

where

$$
a^{\mu}=\frac{1}{2 p^{2}} \epsilon_{\nu \rho \sigma}^{\mu} p^{\nu} S^{\rho \sigma}, \quad(a * \partial)^{\mu}{ }_{\nu} \equiv \epsilon_{\nu \rho \sigma}^{\mu} a^{\rho} \partial^{\sigma},
$$

and $u$ is the four-velocity of the black hole, $u^{\mu}=p^{\mu} / m$. Identities such as

$$
\begin{aligned}
(a * \partial)^{\mu}{ }_{\nu}(a * \partial)^{\nu}{ }_{\rho} \frac{u^{\rho}}{r} & =-(a \cdot \partial)^{2} \delta_{\rho}^{\mu} \frac{u^{\rho}}{r}, \\
(a * \partial)^{\nu}{ }_{\rho} \frac{u^{\rho}}{r} & =S(p)^{\mu}{ }_{\rho} \partial^{\rho} \frac{1}{r}
\end{aligned}
$$

may be used to reorganize the exponential factor.

To compare with our trilinear vertex we need to Fourier transform $\hat{T}^{\mu \nu}$ to momentum space, which is easily done via the substitution $\partial_{\mu} \rightarrow i q_{\mu}$. It leads to

$$
\begin{aligned}
\hat{T}^{\mu \nu} & =m \exp (i a * q)^{(\mu}{ }_{\rho} u^{\nu)} u^{\rho} \\
& =m(\cos a * q+i \sin a * q)^{(\mu} u^{\nu)} u^{\rho} \\
& =m\left(\cosh (a \cdot q) \delta_{\rho}^{(\mu}+i \frac{(a * q)^{(\mu}{ }_{\rho}}{a \cdot q} \sinh (a \cdot q)\right) u^{\nu)} u^{\rho} \\
& =m \cosh (a \cdot q) u^{\mu} u^{\nu}-\frac{i}{a \cdot q} \sinh (a \cdot q) q^{\rho} S(p)_{\rho}{ }^{(\mu} u^{\nu)} .
\end{aligned}
$$

It is not difficult to see, using Eq. (3.14), that the building block of this expression, $a \cdot q$, is the same as the building block of Eq. (3.15):

$$
a \cdot q=\frac{1}{2 m^{2}} \epsilon_{\mu \nu \rho \sigma} q^{\mu} p^{\nu} S(p)^{\rho \sigma}=-\frac{q \cdot S(p)}{m} .
$$

Further using the relation between momentum and velocity, Eqs. (3.22) and (3.15) become identical if we choose 


$$
C_{E S^{2 n}}=1, \quad C_{B S^{2 n}}=1 .
$$

Equation (3.17) then implies that the $\mathrm{H}$ parameter of the Kerr black hole is

$$
\mathrm{H}=1 \text {. }
$$

As mentioned earlier, this value is the one required $[83,84]$ for amplitudes of higher-spin fields to have an improved high-energy behavior delaying violations of partial-wave unitarity.

The relation between the Kerr stress tensor [58] and the one following from the Lagrangian (2.22) and (3.2) implies that the tree-level scattering amplitude of two higher-spin fields - and consequently the 1PM effective Hamiltonianwill also reproduce the scattering amplitude of two Kerr black holes found in Ref. [58]. In subsequent sections we will be interested in Hamiltonian terms that contain at most one spin vector for each of the two particles, so we will focus on the minimal Lagrangian (2.22) and ignore the higherderivative terms in the nonminimal Lagrangian (3.2).

\section{The double-copy properties of general three-point vertex}

For computations beyond leading order in Newton's constant it can be quite useful to exploit the doublecopy structure of gravitational theories. This property played a useful role in the computation of the two-body Hamiltonians for spinless particles at $\mathcal{O}\left(G^{3}\right)$ [14,15].
The double-copy properties of amplitudes of massive spin-1/2 and spin-1 massive particles have been studied in some detail in [76], using the massive-spinor-helicity formalism. Here we make a few observations on the properties of the trilinear vertex for graviton-coupled arbitrary spin particles and in Sec. IV we derive a double-copy formula for the tree-level gravitational Compton amplitude of the minimal Lagrangian. This tree-level amplitude, together with the three- and fourpoint amplitudes of higher-spin particles, is the building block of the one-loop amplitude we construct in Sec. V.

As we now show, for generic values of the parameters $C_{E S^{2 n}}$ and $C_{B S^{2 n}}$, the complete on-shell trilinear gravitonhigher-spin vertex,

$$
V_{3}^{\mu \nu}=V_{3, \min }^{\mu \nu}+V_{3, \text { non- } \min }^{\mu \nu},
$$

can be expressed as the double-copy of trilinear vertices coupling higher-spin fields with vector fields. The double copy is usually formulated in terms of non-Abelian vector fields; for three-point interactions, the non-Abelian structure is not essential, so one may equally well describe (3.26) as the double copy of trilinear vertices coupling higher-spin fields with a Maxwell field. Extension of the double-copy property for four- and higher-point amplitudes is an interesting open question.

To see explicitly these properties, consider the general trilinear vector-higher-spin vertex $^{9}$ arising from the Lagrangian,

$$
\begin{aligned}
\mathcal{L}= & \frac{1}{4} F_{\mu \nu}^{a} F^{a, \mu \nu}-\frac{1}{2} \sum_{n=0}^{\infty} \frac{C_{n} \eta_{\mu_{0} \nu_{0}}}{2^{2 n} m^{4 n}} \epsilon_{\mu_{1} \nu_{1} \rho_{1} \sigma_{1}} \cdots \epsilon_{\mu_{2 n} \nu_{2 n} \rho_{2 n} \sigma_{2 n}} D^{\left(\mu_{0}\right.} D^{\mu_{1}} \cdots D^{\left.\mu_{2 n}\right)} \varphi_{s_{g}} \operatorname{Sym}\left[M^{\rho_{1} \sigma_{1}}, \ldots, M^{\rho_{2 n} \sigma_{2 n}}\right] D^{\left(\nu_{0}\right.} D^{\nu_{1}} \cdots D^{\left.\nu_{2 n}\right)} \varphi_{s_{g}} \\
& +\frac{1}{2} m^{2} \sum_{n=0}^{\infty} \frac{C_{n}}{2^{2 n} m^{4 n}} \epsilon_{\mu_{1} \nu_{1} \rho_{1} \sigma_{1}} \cdots \epsilon_{\mu_{2 n} \nu_{2 n} \rho_{2 n} \sigma_{2 n}} D^{\left(\mu_{1}\right.} \cdots D^{\left.\mu_{2 n}\right)} \varphi_{s_{g}} \operatorname{Sym}\left[M^{\rho_{1} \sigma_{1}}, \ldots, M^{\rho_{2 n} \sigma_{2 n}}\right] D^{\left(\nu_{1}\right.} \cdots D^{\left.\nu_{2 n}\right)} \varphi_{s_{g}} \\
& +\frac{i}{2} \sum_{n=0}^{\infty} \frac{E_{n}}{2^{2 n} m^{4 n}} \epsilon_{\mu_{1} \nu_{1} \rho_{1} \sigma_{1}} \cdots \epsilon_{\mu_{2 n} \nu_{2 n} \rho_{2 n} \sigma_{2 n}} D^{\left(\mu_{1}\right.} \cdots D^{\left.\mu_{2 n}\right)} \varphi_{s_{g}} \operatorname{Sym}\left[M_{\mu_{0} \nu_{0}} F^{\mu_{0} \nu_{0}}, M^{\rho_{1} \sigma_{1}}, \ldots, M^{\rho_{2 n} \sigma_{2 n}}\right] D^{\left(\nu_{2 n}\right.} \cdots D^{\left.\mu_{1}\right)} \varphi_{s_{g}}
\end{aligned}
$$

Here we assume that the real higher-spin field $\varphi_{s_{g}}$ is in a real nonadjoint representation of some gauge group $\mathcal{G}, D^{\nu}$ is the corresponding covariant derivative, and $F_{\mu_{0} \nu_{0}} \equiv$ $F_{\mu_{0} \nu_{0}}^{a} T^{a}$ is its field strength. Equation (3.27) may be given in a (slightly) more compact form in terms of an operator obtained from $\mathbb{S}$ defined in Eq. (3.1) by replacing the gravitational covariant derivative with a gauge-covariant derivative. Both Lorentz and gauge group indices are

\footnotetext{
${ }^{9}$ As in their coupling to gravity, here too higher-spin fields are not required to be transverse.
}

contracted via matrix multiplication. The scalar coefficients $C_{n}$ and $E_{n}$ are arbitrary except for $C_{0}=-1$ defining the quadratic term of the higher-spin field; all quadratic terms with more than two derivatives cancel out upon integration by parts. Last, Lorentz generators $M$ are in the $\left(s_{g}+1, s_{g}+1\right)$ representation; for the purpose of this action one may think of them only as the ClebschGordan coefficients for projection $\left(s_{g}+1, s_{g}+1\right) \times$ $\left(s_{g}+1, s_{g}+1\right) \rightarrow(3,1) \oplus(1,3)$.

The color-stripped three-point vertex can easily be read from the action: 


$$
\begin{aligned}
-i V_{3 a\left(s_{g}\right)}^{\mu_{0}} b\left(s_{g}\right)= & \frac{i}{2}\left(p_{2}-p_{1}\right)^{\mu_{0}} \sum_{n=0}^{\infty} \hat{C}_{n}^{\mu_{1} \nu_{1}, \ldots, \mu_{2 n} \nu_{2 n}}\left(M^{\mu_{1} \nu_{1}} \cdots M^{\mu_{2 n} \nu_{2 n}}\right)_{a\left(s_{g}\right)} b\left(s_{g}\right) \\
& -\sum_{n=0}^{\infty} \hat{E}_{n}^{\mu_{1} \nu_{1}, \ldots, \mu_{2 n} \nu_{2 n}} \operatorname{Sym}\left[M^{\mu_{0} \nu_{0}} q_{\nu_{0}}, M^{\mu_{1} \nu_{1}}, \ldots, M^{\mu_{2 n} \nu_{2 n}}\right]_{a\left(s_{g}\right)} b\left(s_{g}\right) .
\end{aligned}
$$

Here $p_{1}$ and $p_{2}$ are the momenta of the tensor fields with Lorentz indices $b\left(s_{g}\right)$ and $a\left(s_{g}\right)$, respectively, and $q=-p_{1}-p_{2}$ is the gluon momentum. The tensors $\hat{C}_{n}$ and $\hat{E}_{n}$ are symmetric under the interchange of pairs of $\left(\mu_{i} \nu_{i}\right)$ indices and read:

$$
\begin{aligned}
\hat{C}_{n}^{\mu_{1} \nu_{1}, \ldots, \mu_{2 n} \nu_{2 n}} & =\frac{C_{n}}{2^{2 n} m^{4 n}} \epsilon^{\rho_{1} \sigma_{1} \mu_{1} \nu_{1}} q_{\rho_{1}} p_{1 \sigma_{1}} \cdots \epsilon^{\rho_{2 n} \sigma_{2 n} \mu_{2 n} \nu_{2 n}} q_{\rho_{2 n}} p_{1 \sigma_{2 n}}, \\
\hat{E}_{n}^{\mu_{1} \nu_{1}, \ldots, \mu_{2 n} \nu_{2 n}} & =\frac{E_{n}}{2^{2 n} m^{4 n}} \epsilon^{\rho_{1} \sigma_{1} \mu_{1} \nu_{1}} q_{\rho_{1}} p_{1 \sigma_{1}} \cdots \epsilon^{\rho_{2 n} \sigma_{2 n} \mu_{2 n} \nu_{2 n}} q_{\rho_{2 n}} p_{1 \sigma_{2 n}} .
\end{aligned}
$$

In deriving them we ignored terms proportional to the free equations of motion and terms that contain more powers of the gluon momentum than the ones shown. In the contraction of the Levi-Civita symbols, Lorentz generators, and momenta we may recognize the repeated appearance of the operator $\mathbb{S}_{0}$ defined in Eq. (3.5). Up to numerical coefficients, the tensors $\hat{C}_{n}$ and $\hat{E}_{n}$ are proportional, so their product also is totally symmetric in all $\left(\mu_{i} \nu_{i}\right)$ pairs of indices.

To construct the double copy of two such vertices, one with $s_{g}=s_{L}$ and the other with $s_{g}=s_{R}$, we need the projection of the product $\left(s_{L}+1, s_{L}+1\right) \times\left(s_{R}+1, s_{R}+1\right)$ onto $(s+1, s+1)$ with $s=s_{L}+s_{R}$. Using the fact that the double-copy vertex is contracted with a polarization tensor in the $(s+1, s+1)$ representation, the relevant projection (denoted by the vertical bar and realized e.g., by contracting all two-component spinor indices with identical commuting spinors) is

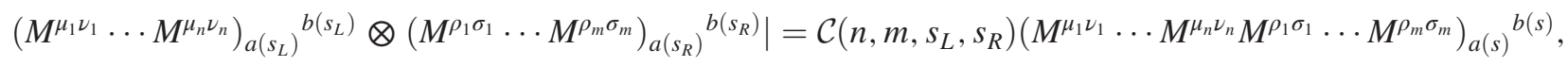

where

$$
\mathcal{C}\left(n, m, s_{L}, s_{R}\right)=\frac{s_{L} !}{\left(s_{L}-n\right) !} \frac{s_{R} !}{\left(s_{R}-m\right) !} \frac{(s-n-m) !}{s !}
$$

Evaluating

$$
-i \varepsilon\left(\boldsymbol{s}, p_{2}\right) V_{3, \mathrm{DC}}^{\mu_{0} \nu_{0}} \varepsilon\left(\boldsymbol{s}, p_{1}\right)=\left[-i \varepsilon\left(\boldsymbol{s}_{L}, p_{2}\right) V_{3, \mathrm{~L}}^{\mu_{0} \nu_{0}} \varepsilon\left(\boldsymbol{s}_{L}, p_{1}\right)\right]\left[-i \varepsilon\left(\boldsymbol{s}_{R}, p_{2}\right) V_{3, \mathrm{R}}^{\mu_{0} \nu_{0}} \varepsilon\left(\boldsymbol{s}_{R}, p_{1}\right)\right]
$$

in the classical limit by using the relation between polarization tensors $\varepsilon(\boldsymbol{s}, p)=\varepsilon\left(\boldsymbol{s}_{L}, p\right) \otimes \varepsilon\left(\boldsymbol{s}_{R}, p\right) \mid$, using the identity (3.16), and ignoring terms that vanish for on-shell gravitons, we find

$$
\begin{aligned}
-i \varepsilon\left(\boldsymbol{s}, p_{2}\right) V_{3, \mathrm{DC}}^{\mu_{0} \nu_{0}} \varepsilon\left(\boldsymbol{s}, p_{1}\right)= & p_{1}^{\mu_{0}} p_{1}^{\nu_{0}} \sum_{n, m=0}^{\infty} \mathcal{C}\left(n, m, s_{L}, s_{R}\right) C_{n} C_{m} \varepsilon\left(\boldsymbol{s}, p_{2}\right)\left(\frac{q \cdot \mathbb{S}_{0}}{m}\right)^{2 m+2 n} \varepsilon\left(\boldsymbol{s}, p_{1}\right) \\
& -p_{1}^{\mu_{0}} p_{1}^{\nu_{0}} \sum_{n, m=0}^{\infty} \mathcal{C}\left(n, m, s_{L}, s_{R}\right) E_{n} E_{m} \varepsilon\left(\boldsymbol{s}, p_{2}\right)\left(\frac{q \cdot \mathbb{S}_{0}}{m}\right)^{2 n+2 m+2} \varepsilon\left(\boldsymbol{s}, p_{1}\right) \\
& -i \sum_{n, m=0}^{\infty} C_{n} E_{m} \varepsilon\left(\boldsymbol{s}, p_{2}\right) \operatorname{Sym}\left[\left(\mathcal{C}\left(n, m, s_{L}, s_{R}\right) p_{1}^{\mu_{0}} M^{\nu_{0} \sigma_{0}} q_{\sigma_{0}}\right.\right. \\
& \left.\left.+\mathcal{C}\left(m, n, s_{L}, s_{R}\right) p_{1}^{\nu_{0}} M^{\mu_{0} \sigma_{0}} q_{\sigma_{0}}\right),\left(\frac{q \cdot \mathbb{S}_{0}}{m}\right)^{2 m+2 n}\right] \varepsilon\left(\boldsymbol{s}, p_{1}\right)
\end{aligned}
$$

If $s_{L} \neq s_{R}$, the expression above contains an antisymmetric part which is identified with a coupling with the Neveu-Schwarz $B$ field. It is not difficult to see that the symmetric part has the same tensor structure as the graviton-higher-spin vertex in Eq. (3.15); moreover, any choice of coefficients $C_{E S^{2 n}}$ and $C_{B S^{2 n}}$ can be reproduced by adjusting the coefficients $C_{n}$ and $E_{n}$.

As a special case of this general relation, we show that the double copy of two minimal couplings of a higher-spin field with a vector yields the gravitational minimal coupling (2.27), including the quadrupole contribution. The former may be found by truncating (3.28) to terms with at most one Lorentz generator (i.e., setting to zero all $C_{n \neq 0}$ and $E_{n \neq 0}$ ): 
$-i V_{a\left(s_{L}\right)}^{b\left(s_{L}\right)}\left(q, p_{1}, p_{2}\right)=i p_{1}^{\mu} \delta_{a\left(s_{L}\right)}^{b\left(s_{L}\right)}-q_{\rho}\left(M^{\mu \rho}\right)_{a\left(s_{L}\right)} b\left(s_{L}\right)$.

Constructing the double-copy vertex (3.32) and projecting onto the $(s+1, s+1)$ representation gives

$$
\begin{aligned}
- & i V_{3, \mathrm{DC} a(s)}^{\mu \nu}{ }^{b(s)}\left(q, p_{1}, p_{2}\right) \\
= & -p_{1}^{\mu} p_{1}^{\nu} \delta_{a(s)}^{b(s)}-\frac{i}{s_{L}+s_{R}}\left(s_{R} q_{\rho} p_{1}^{\mu}\left(M^{\nu \rho}\right)_{a(s)} b(s)\right. \\
& \left.+s_{L} q_{\rho} p_{1}^{\nu}\left(M^{\mu \rho}\right)_{a(s)}^{b(s)}\right) \\
& +\frac{1}{2}\left(2 \frac{s_{L} s_{R}}{s(s-1)}\right) q_{\rho} q_{\sigma}\left(M^{(\mu \mid \rho} M^{\mid \nu) \sigma}\right)_{a(s)} b(s)
\end{aligned}
$$

For $s=s_{L}+s_{R}=1$ as well as for $s_{L}=0$ or $s_{R}=0$ the quadrupole term is not generated. As in the general case, for $s_{L} \neq s_{R}$ the on-shell double-copy three-point vertex $V_{3, \mathrm{DC}}^{\mu \nu}$ contains an antisymmetric part, representing the coupling of the Neveu-Schwarz $B$ field with the higher-spin tensor. The term linear in Lorentz generators in the symmetric part of $V_{3 . \mathrm{DC}}^{\mu \nu}$ is universal, independent on $s_{L}$ and $s_{R}$; this is a reflection of the universality of gravity. It is easy to see that the symmetric part of Eq. (3.35) is the on-shell value of the graviton-higher-spin vertex (2.27) derived from the minimal Lagrangian (2.22), with a gravitational quadrupole coefficient given by

$$
\mathrm{H}=2 \frac{s_{L} s_{R}}{s(s-1)} .
$$

This value is the same as the one found in string theory [84]. Equation (3.36) implies that, in the classical limit, where all spins are large, $H<1 / 2$. For low values of the spins, $\mathrm{H}$ can reach unity.

\section{TREE AMPLITUDES}

One of our goals is to obtain new results for the terms bilinear in spin in the two-body Hamiltonian, through $\mathcal{O}\left(G^{2}\right)$, and to all orders in velocity. The key input that we need is the one-loop two-to-two scattering amplitudes for spinning particles. In turn, the generalized unitarity method [27-31] for constructing loop amplitudes relies on suitable tree-amplitude building blocks. In this section, we describe the ones that will be required in subsequent sections to obtain the desired gravitationally induced interactions of spinning particles. To construct these tree amplitudes we use the arbitrary-spin formalism set up in Sec. II. Here we are interested only in terms linear in the spin of each particle, so the minimal Lagrangian in Eq. (2.22) with $\mathrm{H}=0$ is sufficient; the nonminimal interactions discussed in Sec. III are all quadratic or of higher order in spin and will be useful for future studies.
First, we obtain the tree-level amplitude with four external particles of arbitrary spin. The sole contributing diagram is shown in Fig. 3. It gives us the necessary information to determine the $O(G)$ two-body Hamiltonian. Then, we proceed to obtain the tree-level amplitude which will be used in Sec. V to construct the one-loop amplitude that encodes the spin-orbit and spin-spin interactions at $\mathcal{O}\left(G^{2}\right)$. As noted in Refs. [11,12,17,22] and reviewed in Sec. II A, only a limited number of terms in the one-loop amplitude contribute to the long-range classical potential. They are captured by the unitarity cuts that separate the two matter lines, as illustrated at one-loop in Fig. 4. Thus, to build the relevant parts of the one-loop amplitude, we only need the tree amplitudes contributing to these cuts, i.e., the gravitational analog of the tree-level Compton amplitude, whose diagrams are shown Fig. 5. In this section, we will present these tree amplitudes, constructed using our arbitrary-spin formalism. We also comment on some of their important properties, including generalized on-shell Ward identities and double-copy properties.

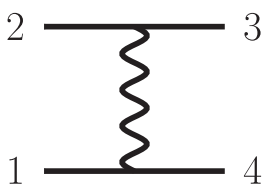

FIG. 3. The tree-level Feynman diagram containing the $\mathcal{O}(G)$ spin interactions. Because we are interested only in long range interactions contact terms where the graviton propagator cancels can be ignored.

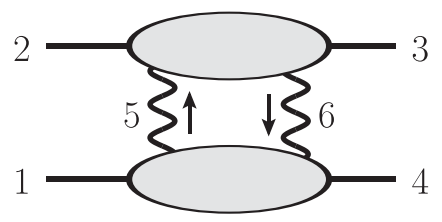

FIG. 4. The two-particle cut needed for extracting classical dynamics. The blobs represent on-shell tree amplitudes and the exposed lines indicate that the propagators are replaced with onshell conditions.

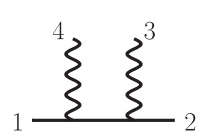

(a)

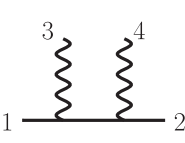

(b)

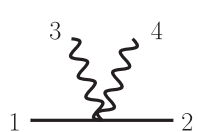

(c)

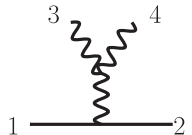

(d)
FIG. 5. The tree-level Feynman diagram for gravitational Compton scattering. For integer-spin electrodynamics diagram (d) is absent. Here the internal lines represent Feynman propagators. 


\section{A. Tree-level two-to-two scattering of spinning particles}

Consider the two-to-two tree-level scattering amplitude encoded in the diagram in Fig. 3 and obtained by sewing together two three-point vertices (2.27) with $\mathrm{H}=0$. We can drop any term that cancels the graviton propagator, since we are only interested in long range interactions. This effectively places the graviton on-shell with the result that each vertex is automatically transverse and thus their contribution to the amplitude is independent of the gauge choice. We may therefore use the relatively simple de Donder gauge propagator,

$$
P_{\text {de Donder }}^{\mu \nu \alpha \beta}(q)=\frac{i}{q^{2}}\left(\frac{1}{2} \eta^{\mu \alpha} \eta^{\nu \beta}+\frac{1}{2} \eta^{\nu \alpha} \eta^{\mu \beta}-\frac{1}{D-2} \eta^{\mu \nu} \eta^{\alpha \beta}\right)
$$

since any longitudinal terms in the graviton physical-state projector are automatically set to zero by the two vertices.

Using the vertex (2.27) and the propagator (4.1) it is then straightforward to obtain the desired tree-level amplitude from the diagram in Fig. 3:

$$
i \mathcal{M}^{\text {tree }}\left(1^{s_{1}}, 2^{s_{2}}, 3^{s_{2}}, 4^{s_{1}}\right)=\varepsilon_{4}{ }^{a(s)} V_{\min a(s)}^{\mu \nu}{ }^{b(s)}\left(q, p_{1}, p_{4}\right) \varepsilon_{1 b(s)} P_{\operatorname{deD} \text { Donder }}^{\mu \nu \alpha \beta}(q) \varepsilon_{3}{ }^{c(s)} V_{\min c(s)}^{\mu \nu} d(s)\left(-q, p_{2}, p_{3}\right) \varepsilon_{2 d(s)},
$$

where $\varepsilon_{i} \equiv \varepsilon\left(s_{i}, p_{i}\right)$ and we can drop any terms that cancel the graviton propagator. Starting with the tree-level vertex given in Eq. (2.27) and defining

$$
M_{i j}(a, b) \equiv \varepsilon\left(p_{i}\right) M^{\mu \nu} \varepsilon\left(p_{j}\right) a_{\mu} b_{\nu}, \quad M_{i j}\left(e^{\mu}, a\right) \equiv \varepsilon_{i} M^{\mu \nu} \varepsilon_{j} a_{\nu},
$$

where $e^{\mu}$ is a unit vector signifying that the $\mu$ index is uncontracted, from Eq. (4.2) we obtain

$$
\begin{aligned}
i \mathcal{M}^{\text {tree }}\left(1^{s_{1}}, 2^{s_{2}}, 3^{s_{2}}, 4^{s_{1}}\right)= & -\frac{16 \pi i G m_{1}^{2} m_{2}^{2}}{q^{2}}\left\{\left(2 \sigma^{2}-1\right) \varepsilon_{1} \cdot \varepsilon_{4} \varepsilon_{2} \cdot \varepsilon_{3}+\frac{2 i \sigma}{m_{1} m_{2}} M_{14}\left(p_{2}, q\right) \varepsilon_{2} \cdot \varepsilon_{3}-\frac{i}{m_{1}^{2}} M_{14}\left(p_{1}, q\right) \varepsilon_{2} \cdot \varepsilon_{3}\right. \\
& -\frac{2 i \sigma}{m_{1} m_{2}} \varepsilon_{1} \cdot \varepsilon_{4} M_{23}\left(p_{1}, q\right)+\frac{i}{m_{2}^{2}} \varepsilon_{1} \cdot \varepsilon_{4} M_{23}\left(p_{2}, q\right) \\
& \left.+\frac{1}{m_{1}^{2} m_{2}^{2}}\left(M_{14}\left(p_{2}, q\right) M_{23}\left(p_{1}, q\right)-M_{14}\left(p_{1}, q\right) M_{23}\left(p_{2}, q\right)\right)-\frac{\sigma}{m_{1} m_{2}} M_{14}\left(e^{\mu}, q\right) M_{23}\left(e^{\nu}, q\right) \eta_{\mu \nu}\right\},
\end{aligned}
$$

where we use the dimensionless kinematic variable,

$$
\sigma \equiv \frac{p_{1} \cdot p_{2}}{m_{1} m_{2}}
$$

In the classical limit, the products of polarization tensors and Lorentz generators (4.3) are related to the spin tensors of the two particles through Eqs. (2.20); thus, the tree amplitude can be expressed solely in terms of them as well as the products $\varepsilon_{i} \cdot \varepsilon_{j}$. Further using Eq. (2.18) and the covariant spin supplementary condition (2.19), the amplitude becomes

$$
\begin{aligned}
i \mathcal{M}^{\text {tree }}\left(1^{s_{1}}, 2^{s_{2}}, 3^{s_{2}}, 4^{s_{1}}\right)= & -\frac{16 \pi i G m_{1} m_{2}}{q^{2}} \varepsilon_{1} \cdot \varepsilon_{4} \varepsilon_{2} \cdot \varepsilon_{3}\left\{m_{1} m_{2}\left(2 \sigma^{2}-1\right)-2 \sigma\left(i S_{2}\left(p_{1}, q\right)-i S_{1}\left(p_{2}, q\right)\right)\right. \\
& \left.-\left(\frac{1}{m_{1} m_{2}} S_{1}\left(p_{2}, q\right) S_{2}\left(p_{1}, q\right)+\sigma S_{1}\left(e^{\mu}, q\right) S_{2}\left(e^{\nu}, q\right) \eta_{\mu \nu}\right)\right\}+\mathcal{O}\left(q, S_{i}^{2}\right),
\end{aligned}
$$

where, in close analogy with Eq. (4.3), we defined

$$
S_{i}(a, b) \equiv a_{\mu} b_{\nu} S^{\mu \nu}\left(p_{i}\right), \quad S_{i}\left(e^{\mu}, b\right) \equiv b_{\nu} S^{\mu \nu}\left(p_{i}\right) .
$$

The parametrization of the classical amplitude in terms of the spin tensor emphasizes its close relation to its complete quantum origin. As we will see in Sec. V, this persists at loop-level and we will organize the amplitude in a similar form, which will have a structure close to that of the unitarity cuts. 
Using Eq. (2.18), and the identities for products of Levi-Civita tensors, we can express the result in terms of the spin vector. During this transformation, terms with more than two powers of the momentum transfer $q$ appearing in the bilinears in spin tensor as well as terms canceling the graviton propagator are discarded. We find

$$
\begin{aligned}
i \mathcal{M}^{\text {tree }}\left(1^{s_{1}}, 2^{s_{2}}, 3^{s_{2}}, 4^{s_{1}}\right)= & -\frac{16 \pi i G m_{1} m_{2}}{q^{2}} \varepsilon_{1} \cdot \varepsilon_{4} \varepsilon_{2} \cdot \varepsilon_{3}\left\{m_{1} m_{2}\left(2 \sigma^{2}-1\right)-2 i \sigma \epsilon^{\mu \nu \rho \sigma} p_{1 \mu} p_{2 \nu} q_{\rho}\left(\frac{S_{1 \sigma}}{m_{1}}+\frac{S_{2 \sigma}}{m_{2}}\right)\right. \\
& \left.+\left(2 \sigma^{2}-1\right) q \cdot S_{1} q \cdot S_{2}\right\}+\mathcal{O}\left(q, S_{i}^{2}\right) .
\end{aligned}
$$

It is straightforward to further write the amplitude in terms of the rest-frame spin vectors of the two particles; we will do this in Sec. VE and will be an important input in the construction of the EFT in Sec. VI.

\section{B. Tree-level gravitational Compton amplitude}

To obtain the tree-level gravitational Compton amplitude needed to construct the one-loop four-point matter amplitude, we follow the same basic procedure. It is obtained by straightforwardly evaluating the four Feynman diagrams in Fig. 5, with two external arbitrary-spin particles and two gravitons. We need to include the contribution of the fourpoint vertex arising from the Lagrangian (2.22) and shown in Fig. 5(c) as it contributes, together with other contact terms from collapsing internal propagators, to the classical part of the loop amplitudes we construct from this tree amplitude.

Once we have obtained the Compton amplitude we put it in a factorized form inspired by the KLT relations [41], which express gravitational amplitudes in terms of gaugetheory ones. First we note that the case of spinless external matter has a simple factorization into a product of amplitudes in scalar electrodynamics,

$$
\begin{aligned}
& i \mathcal{M}\left(1^{0}, 2^{0}, 3^{h}, 4^{h}\right) \\
& \quad=-4 \pi i G \frac{p_{1} \cdot p_{3} p_{1} \cdot p_{4}}{p_{3} \cdot p_{4}}\left[A\left(1^{0}, 2^{0}, 3^{A}, 4^{A}\right)\right]^{2},
\end{aligned}
$$

where $A\left(1^{0}, 2^{0}, 3^{A}, 4^{A}\right)$ is the scalar electrodynamics Compton amplitude, the 0 superscript indicated that the matter leg is spinless, and the $h$ and $A$ superscripts indicate the leg is a graviton and photon, respectively. The factors of $i$ are due to our choices for normalizing the amplitudes. Similarly, the arbitrary-spin amplitude also factorizes into electrodynamics amplitudes,

$$
\begin{aligned}
& i \mathcal{M}\left(1^{s}, 2^{s}, 3^{h}, 4^{h}\right) \\
& =-4 \pi i G \frac{p_{1} \cdot p_{3} p_{1} \cdot p_{4}}{p_{3} \cdot p_{4}} A\left(1^{0}, 2^{0}, 3^{A}, 4^{A}\right) A\left(1^{s}, 2^{s}, 3^{A}, 4^{A}\right),
\end{aligned}
$$

where the second amplitude $A\left(1^{s}, 2^{s}, 3^{A}, 4^{A}\right)$ is an electrodynamics Compton amplitude for an arbitrary-spin particle as indicated by the superscript $s$. In Eq. (4.10) the graviton polarization tensor is identified in terms of a product of two photon polarizations,

$$
\varepsilon^{\gamma_{1} \gamma_{2}}\left(p_{3}\right)=\varepsilon^{\gamma_{1}}\left(p_{3}\right) \mathcal{E}^{\gamma_{2}}\left(p_{3}\right) .
$$

While inspired by KLT factorization, Eq. (4.10) differs somewhat from the usual field theory KLT relation in two ways: it holds for arbitrary-spin massive particles, and the factorization involves Abelian rather than non-Abelian gauge-theory amplitudes.

The scalar electrodynamics amplitude $A\left(1^{0}, 2^{0}, 3^{A}, 4^{A}\right)$ is derived from the standard Lagrangian,

$$
\mathcal{L}_{s=0, \mathrm{EM}}=-\frac{1}{4} F^{\mu \nu} F_{\mu \nu}+D_{\mu}^{\dagger} \bar{\phi} D^{\mu} \phi-m^{2} \bar{\phi} \phi,
$$

where $F_{\mu \nu}$ is the usual Maxwell field strength and $D^{\mu}$ the corresponding covariant derivative. Similarly, the arbitraryspin electromagnetic Compton amplitude in Eq. (4.10) arises from the Lagrangian with a gyromagnetic ratio, $g=2$,

$$
\begin{aligned}
\mathcal{L}_{s, \mathrm{EM}}= & -\frac{1}{4} F^{\mu \nu} F_{\mu \nu}+D_{\mu}^{\dagger} \bar{\phi}_{s} D^{\mu} \phi_{s}-m^{2} \bar{\phi}_{s} \phi_{s} \\
& +e(g-1) F_{\mu \nu} \bar{\phi}_{s} M^{\mu \nu} \phi_{s},
\end{aligned}
$$

where $M$ is a Lorentz generator. We suppress the Lorentz indices of the higher-spin fields, as in Eq. (2.22). Because we are coupling to a $U(1)$ gauge field we take the higherspin field $\phi_{s}$ to be complex here and (4.13) is the complex version of the two-derivative truncation of the Lagrangian (3.27) used in Sec. III C to show that the minimal higherspin-graviton vertex has a double-copy structure. ${ }^{10}$ As discussed there, the $\mathrm{H}$ (quadrupole) term is not generated if one of the two spins vanishes, which is consistent with the left-hand side of Eq. (4.10) arising from the Lagrangian (2.22) with $\mathrm{H}=0$.

To present the explicit form of the amplitudes we strip them of their external polarization vectors and tensors,

\footnotetext{
${ }^{10}$ Equation (4.13) is also a rewriting of Eq. (3.27) for an $S O(2)$ gauge group and higher-spin matter fields in its vector representation.
} 


$$
A\left(1^{s}, 2^{s}, 3^{A}, 4^{A}\right)=\varepsilon_{\alpha}\left(p_{3}\right) \varepsilon_{\beta}\left(p_{4}\right) \varepsilon_{s}\left(p_{1}\right) \cdot A^{\alpha \beta}\left(1^{s}, 2^{s}, 3^{A}, 4^{A}\right) \cdot \varepsilon_{s}\left(p_{2}\right),
$$

where as usual we suppress the higher-spin indices for legs 1 and 2. The dot products refer to the contraction of these indices. For spinless matter fields we have

$$
A\left(1^{0}, 2^{0}, 3^{A}, 4^{A}\right) \equiv \varepsilon_{\alpha}\left(p_{3}\right) \varepsilon_{\beta}\left(p_{4}\right) A^{\alpha \beta}\left(1^{0}, 2^{0}, 3^{A}, 4^{A}\right) .
$$

The explicit polarization-stripped amplitude is

$$
\begin{aligned}
A^{\alpha \beta}\left(1^{s}, 2^{s}, 3^{A}, 4^{A}\right)= & A^{\alpha \beta}\left(1^{0}, 2^{0}, 3^{A}, 4^{A}\right) \mathbb{1}-2 i\left\{\left(\frac{p_{1}^{\beta}}{p_{1} \cdot p_{4}}-\frac{p_{2}^{\beta}}{p_{2} \cdot p_{4}}\right) p_{3 \gamma} i M^{\gamma \alpha}+\left(\frac{p_{1}^{\alpha}}{p_{1} \cdot p_{3}}-\frac{p_{2}^{\alpha}}{p_{2} \cdot p_{3}}\right) p_{4 \delta} i M^{\delta \beta}\right. \\
& \left.-p_{3 \gamma} p_{4 \delta}\left(\frac{1}{p_{1} \cdot p_{4}} M^{\alpha \gamma} M^{\beta \delta}+\frac{1}{p_{1} \cdot p_{3}} M^{\beta \delta} M^{\alpha \gamma}\right)\right\}
\end{aligned}
$$

where we have suppressed the indices of the higher-spin fields, $M$ is a Lorentz generator and $\mathbb{1}$ is the identity matrix of the $\left(s_{1}+1, s_{2}+1\right)$ representation with $s=s_{1}+s_{2}, g=2$, and we have dropped the electromagnetic coupling constant $e$. The scalar part is

$$
A^{\alpha \beta}\left(1^{0}, 2^{0}, 3^{A}, 4^{A}\right)=2 i\left(\frac{p_{1}^{\beta} p_{2}^{\alpha}}{p_{1} \cdot p_{4}}+\frac{p_{1}^{\alpha} p_{2}^{\beta}}{p_{1} \cdot p_{3}}+\frac{p_{3}^{\alpha} p_{3}^{\beta}+p_{4}^{\alpha} p_{4}^{\beta}}{p_{3} \cdot p_{4}}+\eta^{\alpha \beta}\right)
$$

From Eq. (4.10) the arbitrary-spin polarization-stripped gravitational Compton amplitude [derived from the Lagrangian (2.22) with $\mathrm{H}=0$ ] is

$$
i \mathcal{M}^{\gamma_{1} \gamma_{2}, \delta_{1} \delta_{2}}\left(1^{s}, 2^{s}, 3^{h}, 4^{h}\right)=-4 \pi i G \frac{p_{1} \cdot p_{3} p_{1} \cdot p_{4}}{p_{3} \cdot p_{4}} A^{\gamma_{1} \delta_{1}}\left(1^{0}, 2^{0}, 3^{A}, 4^{A}\right) A^{\gamma_{2} \delta_{2}}\left(1^{s}, 2^{s}, 3^{A}, 4^{A}\right) .
$$

Similarly, the case of spinless matter can be written as

$$
i \mathcal{M}^{\gamma_{1} \gamma_{2}, \delta_{1} \delta_{2}}\left(1^{0}, 2^{0}, 3^{h}, 4^{h}\right)=-4 \pi i G \frac{p_{1} \cdot p_{3} p_{1} \cdot p_{4}}{p_{3} \cdot p_{4}} A^{\gamma_{1} \delta_{1}}\left(1^{0}, 2^{0}, 3^{A}, 4^{A}\right) A^{\gamma_{2} \delta_{2}}\left(1^{0}, 2^{0}, 3^{A}, 4^{A}\right)
$$

In practice, the spinless limit follows simply by setting the Lorentz generators $M$ to zero, and the scalar products of massiveparticle polarization tensors $\varepsilon(\boldsymbol{s}, p)$ to be unity.

The KLT-inspired form of the gravitational amplitudes inherits useful properties directly from the photon amplitudes. Specifically, the spin- 0 and spin- $s$ electromagnetic Compton amplitudes are automatically transverse on each photon leg, without the need contracting the other legs with polarization tensors. From Eqs. (4.16) and (4.17) it is straightforward to verify that, for any $s$ including $s=0$,

$$
p_{3 \gamma} A^{\gamma \delta}\left(1^{s}, 2^{s}, 3^{A}, 4^{A}\right)=0, \quad p_{4 \delta} A^{\gamma \delta}\left(1^{s}, 2^{s}, 3^{A}, 4^{A}\right)=0,
$$

using only the antisymmetry of the Lorentz matrices and the on-shell conditions for all external momenta. The net effect is that, when sewing photon lines of amplitudes with this property, physical-state projectors are not required. Note that the terms depending on $p_{3}$ and $p_{4}$ in Eq. (4.17), which vanish when contracted with physical gluon polarization vectors, are crucial to ensure this property.

The gravity amplitudes stripped of polarization tensors automatically inherit similar Ward identities via the KLT-like relation (4.10). Together with Eq. (4.20), this implies that

$$
\begin{aligned}
& p_{3 \gamma_{1}} \mathcal{M}^{\gamma_{1} \gamma_{2}, \delta_{1} \delta_{2}}\left(1^{s}, 2^{s}, 3^{h}, 4^{h}\right)=0, \quad p_{3 \gamma_{2}} \mathcal{M}^{\gamma_{1} \gamma_{2}, \delta_{1} \delta_{2}}\left(1^{s}, 2^{s}, 3^{h}, 4^{h}\right)=0, \\
& p_{4 \delta_{1}} \mathcal{M}^{\gamma_{1} \gamma_{2}, \delta_{1} \delta_{2}}\left(1^{s}, 2^{s}, 3^{h}, 4^{h}\right)=0, \quad p_{4 \delta_{2}} \mathcal{M}^{\gamma_{1} \gamma_{2}, \delta_{1} \delta_{2}}\left(1^{s}, 2^{s}, 3^{h}, 4^{h}\right)=0 .
\end{aligned}
$$

Polarization-stripped gravitational amplitudes constructed through standard methods will not automatically satisfy these identities. Typically, on-shell Ward identities hold only after transversality is imposed on all other legs by contracting them with physical state polarization. The differences between such generic forms of polarization-stripped amplitudes and those obeying the generalized on-shell Ward identities (4.21) are terms that vanish upon contraction with the physical polarization 
tensors. An advantage of using amplitudes obeying the generalized form of Ward identities is that the graviton physical state projectors used to sew tree amplitudes into loops reduces to the simple de Donder gauge one (4.1), without requiring ghosts. This in turn simplifies $D$-dimensional cut constructions of loop amplitudes, recently exploited in Refs. [14,15,17].

\section{Connection to field theory KLT relations}

Although the original form of the relations was given for all string theory states, including the massive arbitrary-spin ones, the field theory KLT relations are usually formulated in terms of massless states of spin $s \leq 2$ on the gravitational side. The amplitudes appearing in the two factors are those of non-Abelian Yang-Mills theories (perhaps coupled to additional matter). Here, Eq. (4.10) holds for a single massive particle of arbitrary spin and involves Abelian amplitude factors. Thus, while there is a strong similarity between the double-copy relation (4.10) and the celebrated KLT relations [41], they are not identical. It is therefore worth commenting on the precise connection.

To understand this, we will construct the gravitational Compton amplitude for massive scalars, by dimensionally reducing the standard massless $D$-dimensional KLT relations to four dimensions. This will yield the amplitude in a form that will allow us to connect its factors to the amplitudes of massive scalar electrodynamics that enter Eq. (4.9).

The $D$-dimensional four-graviton tree-level amplitude in KLT form is [41]

$$
\begin{aligned}
& i \mathcal{M}\left(1^{h}, 2^{h}, 3^{h}, 4^{h}\right) \\
& \quad=-16 \pi i G p_{1} \cdot p_{4} A^{\mathrm{YM}}\left(1^{g}, 2^{g}, 3^{g}, 4^{g}\right) A^{\mathrm{YM}}\left(1^{g}, 3^{g}, 2^{g}, 4^{g}\right),
\end{aligned}
$$

where the YM label indicates that these are color-ordered [24] amplitudes of Yang-Mills theory. The superscript $g$ indicates that the leg is a gluon, while the superscript $h$ indicates that it is a graviton. Using the four-point BCJ amplitude relation $[42,86]$ between partial amplitudes,

$$
A^{\mathrm{YM}}\left(1^{g}, 2^{g}, 3^{g}, 4^{g}\right)=\frac{p_{1} \cdot p_{3}}{p_{3} \cdot p_{4}} A^{\mathrm{YM}}\left(1^{g}, 3^{g}, 2^{g}, 4^{g}\right),
$$

allows us to rewrite the four-graviton amplitude as

$$
\begin{aligned}
& i \mathcal{M}\left(1^{h}, 2^{h}, 3^{h}, 4^{h}\right) \\
& \quad=-16 \pi i G \frac{p_{1} \cdot p_{3} p_{1} \cdot p_{4}}{p_{3} \cdot p_{4}}\left[A^{\mathrm{YM}}\left(1^{g}, 3^{g}, 2^{g}, 4^{g}\right)\right]^{2} .
\end{aligned}
$$

The components of the vector fields in the extra $(D-4)$ dimensions appear as scalars in four dimensions. Moreover, the components of the momentum in the extra dimensions acts as a mass for the four-dimensional particles. Thus, in both gauge theory amplitudes, we will choose gluons 3 and 4 to be vectors in four dimensions, with no momenta in the extra dimensions and particles 1 and 2 to be scalars-i.e., vectors pointing in the extra dimensions. We will also assume that they have momentum components in the extra dimensions, so they are massive from a four-dimensional standpoint. [See, for example, Eqs. (3.3) and (3.4) of Ref. [15] for more details.] Thus, the KLT relation for two massive scalars and a two-graviton amplitude is

$$
\begin{aligned}
& i \mathcal{M}\left(1^{0}, 2^{0}, 3^{h}, 4^{h}\right) \\
& \quad=-16 \pi i G \frac{p_{1} \cdot p_{3} p_{1} \cdot p_{4}}{p_{3} \cdot p_{4}}\left[A^{\mathrm{YM}}\left(1^{0}, 3^{g}, 2^{0}, 4^{g}\right)\right]^{2} .
\end{aligned}
$$

This is now of a similar form as Eq. (4.9) except that it is in terms of non-Abelian gauge-theory amplitudes instead of electrodynamics. This difference is inconsequential because the color-ordered diagrams that contribute to the particular color ordering in Eq. (4.25) and collected in Fig. 6 do not contain a three-gluon interaction. They are therefore the same as Maxwell amplitudes, after accounting for different normalizations and signs from reordering the diagrams. With standard normalization of color generators used to define the color-order gauge-theory amplitudes in the KLT relation, one must divide by a factor of $\sqrt{2}$ for each factor of the electric charge and account for color ordering signs,

$$
A^{\mathrm{YM}}\left(1^{0}, 3^{g}, 2^{0}, 4^{g}\right)=-\frac{1}{2} A^{\mathrm{EM}}\left(1^{0}, 2^{0}, 3^{A}, 4^{A}\right),
$$

where the legs on the left-hand side are ordered and on the right-hand side are unordered. Thus, we obtain

$$
\begin{aligned}
& i \mathcal{M}\left(1^{0}, 2^{0}, 3^{h}, 4^{h}\right) \\
& \quad=-4 \pi i G \frac{p_{1} \cdot p_{3} p_{1} \cdot p_{4}}{p_{3} \cdot p_{4}}\left[A^{\mathrm{EM}}\left(1^{0}, 2^{0}, 3^{A}, 4^{A}\right)\right]^{2},
\end{aligned}
$$

in agreement with Eq. (4.10).

The same discussion extends straightforwardly to the tree-level scattering amplitude of two spin-1 particles and two gravitons, offering a simple proof of Eq. (4.10) for $s=1$. We note that, as verified by explicitly computing

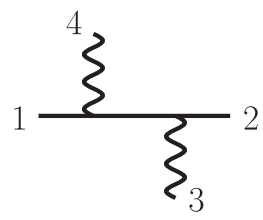

(a)

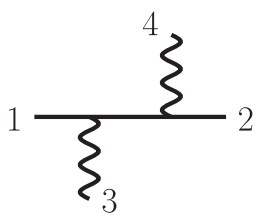

(b)

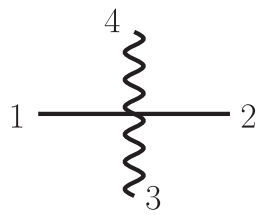

(c)
FIG. 6. The color ordered tree-level Yang-Mills Compton Feynman diagrams, with ordering 1, 3, 2, 4, where legs 1 and 2 are bosonic particles of spin $s$. 
both the gravitational and the electromagnetic Compton amplitudes arising, respectively, from the Lagrangians in Eqs. (2.22) and (4.13), the factorization (4.10) requires that the electromagnetic amplitudes include a magnetic moment coupling, as indicated in Eq. (4.13). This mirrors the situation for the three-point vertex discussed in Sec. III C, where such a coupling was necessary to generate the complete higher-spin-graviton three-point vertex. It is an interesting problem to explore such relations in general, especially at arbitrary orders of the spin and in the presence of higher-dimension operators as in Eq. (3.27). They should become important at higher orders, where they will help simplify calculations and expose new structures.

\section{ONE-LOOP AMPLITUDES}

Using the generalized unitarity method [27], we now construct the parts of the one-loop amplitude needed to extract the classical interaction potential between spinning particles. As reviewed at length in Ref. [15], not all generalized cuts contain useful information about the classical limit of the amplitude. (See also Refs. [11,12, 17,22].) Since we are interested only in long-range interactions, four-point matter contact interactions can be dropped; this implies that whenever graviton propagators that connect the two matter lines are canceled by numerator factors they can be set to zero. In the generalized unitarity language this implies that the contributing terms must have two cut graviton legs separating the two matter lines, as illustrated in Fig. 4. Moreover, the fact that we are scattering classical particles requires that each loop must contain at least one matter line; thus, the cuts that contribute to the classical limit of the amplitude must contain at least one cut matter line per loop. This implies an integrand containing the contributions that we are interested in can be obtained from the quadruple and triple cuts in Figs. 7 and 8. As we describe below, the quadruple-cut contributions correspond to iteration contributions. The triple cuts contain the classical pieces we wish to obtain.

After an integrand is constructed from the unitarity constraints, we apply standard integral reduction methods to express it as a linear combination of scalar integrals. Using these methods any one-loop amplitude can be organized into a linear combination of scalar box, triangle, bubble, and tadpole integrals [87]. We immediately drop any bubble and tadpole integrals that result from the

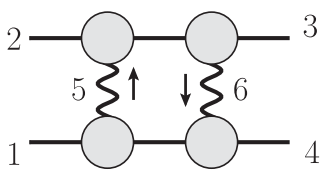

(a)

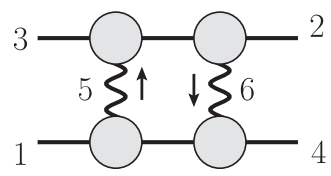

(b)
FIG. 7. The quadruple cut from which the coefficients of the two box integrals in Fig. 9 are extracted. All four external lines are placed on shell.

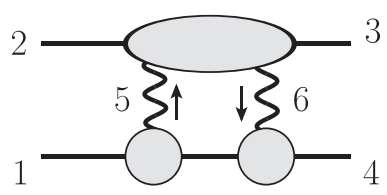

(a)

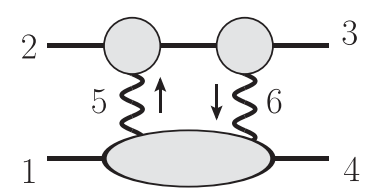

(b)
FIG. 8. The triple cuts from which the coefficient of the triangle integrals are extracted. In each case the three exposed lines are placed on shell.

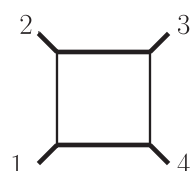

(a)

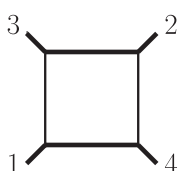

(b)

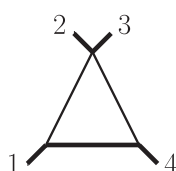

(c)

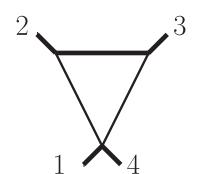

(d)
FIG. 9. (a), (b) The one-loop scalar box integrals that contribute to iterations, and (c), (d) the triangle integrals that contribute to $\mathcal{O}\left(G^{2}\right)$ terms in the potential.

reduction because they are not relevant in the classical limit, leaving only the box and triangle integrals in Fig. 9. Not only do bubble and tadpoles not have the required unitarity cuts, but a direct inspection of the explicit values of the integrals [88] reveals that their dependence on the transferred momentum $q$ is inconsistent with classical dependence while the rational prefactor cannot compensate.

Thus, the classically relevant part of the four-point amplitude is a linear combination of box, $I_{\mathrm{B}}$ and $I_{\overline{\mathrm{B}}}$, and triangle, $I_{\triangle}$ and $I_{\nabla}$, integrals, shown in Figs. 9(a) and 9(b), and Figs. 9(c) and 9(d), respectively:

$$
i \mathcal{M}_{4}^{1 \text { loop }}=d_{\mathrm{B}} I_{\mathrm{B}}+d_{\overline{\mathrm{B}}} I_{\overline{\mathrm{B}}}+c_{\triangle} I_{\triangle}+c_{\nabla} I_{\nabla}
$$

The coefficients $d_{\mathrm{B}}, d_{\overline{\mathrm{B}}}, c_{\triangle}$, and $c_{\nabla}$ are rational functions of external momenta, polarization tensors, and Lorentz generators. In the classical limit the latter can be converted to spin tensors and vectors through an appropriate choice of polarization tensors, using the relations in Sec. II B. The evaluation of scalar integrals in the classical limit is straightforward $[11,12,17,33]$. In any case, since the oneloop Feynman integrals are known [88], we can also simply extract the classical limit directly from these.

One issue that we encounter is that because the box integrals have a stronger-than-classical behavior, subleading terms in the relation between polarization and spin tensors are required for a consistent construction of the classical limit and extraction of the classical interaction potential. Whenever this issue arises we postpone introducing the classical spin tensors until Sec. VI. It turns out that simply by matching the infrared divergences between the amplitudes of the full theory and those of the effective field theory for spinning particles, we find that the physics is insensitive to these subtleties at this order in $G$. 
Well-developed methods for extracting the coefficient of the basics scalar integrals exist for extracting them [30,31] directly from generalized unitarity cuts [27,29]. We use the method due to Forde [31], as extended to massive particles in Ref. [74]. In this method the coefficient of scalar box integrals are computed from quadruple cuts, i.e., cuts in which four internal propagators are replaced with on-shell conditions, as illustrated in Fig. 7. The coefficients of triangle integrals are then extracted from the triple cuts, as illustrated in Fig. 8, in which three internal propagators are replaced with on-shell conditions. While there are alternative ways to carry out the integral reduction to the basis of scalar integrals, this method naturally maintains an organized structure for the coefficients of the integrals even for high-rank numerator tensors of the type that are encountered in multispin interactions of higher-spin fields described in Secs. III A and III B.

\section{A. Sewing tree amplitudes}

The triple and quadruple cuts, shown in Fig. 8 and Fig. 7 , respectively, that are necessary for constructing the coefficients of the scalar integrals in Eq. (5.1) can all be obtained from a regular $t$-channel two-particle cut, shown in Fig. 4. Given that the required gravitational Compton amplitudes are relatively simple, this two-particle cut is a convenient starting point for obtaining the quadruple and triple cuts. To do so one simply replaces additional propagators with on-shell conditions.

We carry out the calculation in $D$ dimensions. Although it is generally more efficient to use four-dimensional tree amplitudes in the unitarity cuts, $D$-dimensional cuts make it straightforward to implement dimensional regularization and thus identify all infrared singularities. This is particularly useful at higher loops, as one needs to ensure that no terms are missed due to subtleties or incomplete handling of dimensional regularization [15]. At one-loop it is not difficult to show that the difference between four- and $D$-dimensional methods for construction of the integrand amounts to certain rational terms in the amplitude [28] that do not have the correct scaling at small transferred momentum to contribute to the classical limit. In particular, they do not have the characteristic $1 / \sqrt{-q^{2}}$ behavior that arises from triangle integrals $[11,33]$.

The two-particle cut corresponding to Fig. 4 is given by

$$
\begin{aligned}
C_{2}= & \sum_{\lambda \lambda^{\prime}} \varepsilon\left(k_{5}\right)_{\lambda}^{\gamma_{1} \gamma_{2}} \varepsilon\left(-k_{6}\right)_{-\lambda^{\prime}}^{\delta_{1} \delta_{2}} M_{\gamma_{1} \gamma_{2} \delta_{1} \delta_{2}}\left(4^{s_{1}}, 1^{s_{1}}, 5^{h},-6^{h}\right) \\
& \times \varepsilon\left(-k_{5}\right)_{-\lambda}^{\gamma_{1}^{\prime} \gamma_{2}^{\prime}} \varepsilon\left(k_{6}\right)_{\lambda^{\prime}}^{\delta_{1}^{\prime} \delta_{2}^{\prime}} M_{\gamma_{1}^{\prime} \gamma_{2}^{\prime} \delta_{1}^{\prime} \delta_{2}^{\prime}}\left(2^{s_{2}}, 3^{s_{2}},-5^{h}, 6^{h}\right),
\end{aligned}
$$

where the two tree amplitudes correspond to the two blobs in that figure and the sum runs over the physical polarization of the cut gravitons. They may be expressed naturally in terms of the sum $\mathcal{P}^{\mu \nu}(k)$ over the physical polarizations of a vector field, as

$\sum_{\lambda} \varepsilon(k)_{\lambda}^{\mu \nu} \varepsilon(-k)_{-\lambda}^{\alpha \beta}=\frac{1}{2} \mathcal{P}^{\mu \alpha} \mathcal{P}^{\nu \beta}+\frac{1}{2} \mathcal{P}^{\nu \alpha} \mathcal{P}^{\mu \beta}-\frac{1}{D-2} \mathcal{P}^{\mu \nu} \mathcal{P}^{\alpha \beta}$,

where

$$
\mathcal{P}^{\mu \nu}(k)=\eta^{\mu \nu}-\frac{r^{\mu} k^{\nu}+r^{\nu} k^{\mu}}{r \cdot k}
$$

and $r^{\mu}$ is an arbitrary null reference vector which should drop out of physical expressions.

The appearance of terms dependent on this reference vector in intermediate expressions complicates the evaluation of the generalized unitarity cut, especially at higher loops. Even at one-loop it is best to eliminate them as early as possible. Because our tree-level amplitudes satisfy, by construction, the on-shell generalized Ward identities (4.21), these terms automatically drop out from the physical-state projectors because in every such term the graviton momentum contracts with a manifestly transverse amplitude. Thus the completeness relation (5.3) reduces to the numerator of the graviton propagator in de Donder gauge,

$$
\begin{aligned}
\sum_{\lambda} \varepsilon(k)_{\lambda}^{\mu \nu} \varepsilon(-k)_{-\lambda}^{\alpha \beta} & =\frac{1}{2} \eta^{\mu \alpha} \eta^{\nu \beta}+\frac{1}{2} \eta^{\nu \alpha} \eta^{\mu \beta}-\frac{1}{D-2} \eta^{\mu \nu} \eta^{\alpha \beta} \\
& \equiv \mathcal{P}_{\text {de Donder }}^{\mu \nu \alpha \beta}
\end{aligned}
$$

A key difference between our construction and the usual de Donder gauge Feynman diagram approach is that here, despite the appearance of the same projector, only physical states propagate so ghosts are not necessary to remove unphysical degrees of freedom. Combining everything, the two-graviton cut in Eq. (5.2) becomes

$$
\begin{aligned}
C_{2}= & M_{\gamma_{1} \gamma_{2} \delta_{1} \delta_{2}}\left(4^{s_{1}}, 1^{s_{1}}, 5^{h},-6^{h}\right) \mathcal{P}_{\text {de Donder }}^{\gamma_{1} \gamma_{2} \gamma_{1}^{\prime} \gamma_{2}^{\prime}} \mathcal{P}_{\text {de Donder }}^{\delta_{1} \delta_{2} \delta_{1}^{\prime} \delta_{2}^{\prime}} \\
& \times M_{\gamma_{1}^{\prime} \gamma_{2}^{\prime} \delta_{1}^{\prime} \delta_{2}^{\prime}}\left(2^{s_{2}}, 3^{s_{2}},-5^{h}, 6^{h}\right),
\end{aligned}
$$

where the superscripts $s_{1}$ and $s_{2}$ indicate the spin of the massive particles and the superscript $h$ indicates that the legs are gravitons. The sewing of tree amplitudes with the de Donder projector (5.5) substantially simplifies the evaluation of generalized cuts at both one and higher loops.

The on-shell conditions for the external and cut legs,

$$
k_{5}^{2}=0, \quad k_{6}^{2} \equiv\left(k_{5}+q\right)^{2}=0,
$$

alter the naive scaling in the limit of small momentum transfer, defined as $q \equiv p_{2}+p_{3}$. We use the momentum assignment in Fig. 8. Indeed, they imply that 


$$
p_{2} \cdot q=q^{2} / 2, \quad p_{1} \cdot q=-q^{2} / 2, \quad k_{5} \cdot q=-q^{2} / 2 .
$$

This improved scaling further simplifies the generalized cuts in the classical limit and, consequently, also the box and triangle coefficients in Eq. (5.1).

\section{B. Quadruple and triple cuts}

The two-graviton cut (5.6), obtained by sewing two treelevel gravitational Compton amplitudes, leads to a rational function of momentum invariants and polarization tensors, whose numerator depends explicitly on loop momenta. We use the entirely algebraic formalism of Refs. [31,74], which extracts the coefficients of box and triangle scalar integrals from quadruple and triple cuts, respectively, shown in Figs. 7 and 8. They in turn are straightforwardly obtained by imposing two and one additional cut conditions on the two-particle cut (5.6), where the input tree amplitudes for capturing the terms bilinear in spin are given in Eq. (4.4).

\section{Quadruple cuts}

The quadruple cut corresponding to Fig. 7(a), which determines the coefficient $d_{\mathrm{B}}$ in Eq. (5.1), is obtained from Eq. (5.6) by cutting the two matter lines carrying momenta $p_{1}+k_{5}$ and $p_{2}-k_{5}$. It is therefore defined as

$$
\left.C_{4}^{(\mathrm{a})} \equiv\left(-2 i p_{1} \cdot k_{5}\right)\left(2 i p_{2} \cdot k_{5}\right) C_{2}\right|_{p_{1} \cdot k_{5} \rightarrow 0, p_{2} \cdot k_{5} \rightarrow 0} .
$$

Because the scalar box integral is more singular in the classical limit than the expected classical terms, it is necessary that the classical limit be taken carefully, by keeping subleading terms in the relation between Lorentz generators and spin tensors. Their details are correlated to the choice of effective field theory that we use to construct the effective interaction Hamiltonian. We therefore temporarily postpone the classical limit and list here the spin-independent terms linear and quadratic in Lorentz generators.

To shorten the expressions we anticipate that each Lorentz generator yields a factor of the spin tensor and therefore has a leading $|q|^{-1}$ scaling in the classical limit. The same expectation allows us to use the covariant spin supplementary condition in the form $M_{14}\left(p_{1}, e^{\mu}\right)=$ $M_{23}\left(p_{2}, e^{\mu}\right)=0$. This clearly holds to leading order in the classical limit [cf. Eq. (2.20)]; we also verify that the needed subleading terms do not spoil this relation. Last but not least, we also take the loop momentum to scale as $k_{5}^{2} \sim q^{2}$ at small $|q|$. We will verify all these expectations in Sec. V C and emphasize that they are not a necessary step in the construction of the (classical limit of the) amplitude. At one-loop it is simple enough to confirm any assumption, by direct computation.

Thus, the part of the quadruple cut independent of Lorentz generators is

$$
\frac{C_{4}^{(\mathrm{a}), S_{i}=0}}{64 \pi^{2} G^{2} m_{1} m_{2}}=4 m_{1}^{3} m_{2}^{3}\left(2 \sigma^{2}-1\right)^{2} \varepsilon_{1} \cdot \varepsilon_{4} \varepsilon_{2} \cdot \varepsilon_{3} .
$$

The momentum dependence reproduces that of the classical limit of the quadruple cuts of the one-loop four-point scattering amplitude of scalar fields [10-12,17,36].

The part of the quadruple cut that is linear in Lorentz generators is

$$
\begin{aligned}
\frac{C_{4}^{(\mathrm{a}), \mathrm{SO}}}{64 \pi^{2} G^{2} m_{1} m_{2}}= & -8 i m_{1}^{2} m_{2}^{2} \sigma\left(2 \sigma^{2}-1\right) M_{14}\left(p_{2}, q\right) \varepsilon_{2} \cdot \varepsilon_{3}+8 i m_{1}^{2} m_{2}^{2} \sigma\left(2 \sigma^{2}-1\right) M_{23}\left(p_{1}, q\right) \varepsilon_{1} \cdot \varepsilon_{4} \\
& +2 i\left(4 m_{1}^{2} m_{2}^{2} \sigma\left(2 \sigma^{2}-1\right)+m_{1} m_{2}^{3}\left(4 \sigma^{2}-1\right)\right) M_{14}\left(k_{5}, q\right) \varepsilon_{2} \cdot \varepsilon_{3} \\
& +2 i\left(4 m_{1}^{2} m_{2}^{2} \sigma\left(2 \sigma^{2}-1\right)+m_{1}^{3} m_{2}\left(4 \sigma^{2}-1\right)\right) M_{23}\left(k_{5}, q\right) \varepsilon_{1} \cdot \varepsilon_{4}
\end{aligned}
$$

where the notation $M_{i j}(a, b)$ is defined in Eq. (4.3). These terms will yield the spin-orbit terms in the coefficients of scalar box integrals.

Finally, the part of the quadruple cut that is quadratic in Lorentz generators with each matter line carrying at least one of them is

$$
\begin{aligned}
\frac{C_{4}^{S_{1} S_{2}}}{64 \pi^{2} G^{2} m_{1} m_{2}}= & -8 m_{1} m_{2}\left(2 \sigma^{2}+1\right) M_{14}\left(k_{5}, p_{2}\right) M_{23}\left(k_{5}, p_{1}\right)+4 m_{1} m_{2}\left(2 \sigma^{2}-1\right) M_{14}\left(p_{2}, q\right) M_{23}\left(p_{1}, q\right) \\
& +\left(8 m_{2}^{2} \sigma+4 m_{1} m_{2}\left(2 \sigma^{2}+1\right)\right) M_{14}\left(k_{5}, q\right) M_{23}\left(k_{5}, p_{1}\right) \\
& -\left(8 m_{1}^{2} \sigma+4 m_{1} m_{2}\left(2 \sigma^{2}+1\right)\right) M_{14}\left(k_{5}, p_{2}\right) M_{23}\left(k_{5}, q\right) \\
& -4 m_{1} m_{2}\left(2 \sigma^{2}+1\right) M_{14}\left(p_{2}, q\right) M_{23}\left(k_{5}, p_{1}\right)-4 m_{1} m_{2}\left(2 \sigma^{2}+1\right) M_{14}\left(k_{5}, p_{2}\right) M_{23}\left(p_{1}, q\right) \\
& +4 m_{1} m_{2}\left(3 \sigma^{2}-1\right) M_{14}\left(p_{2}, q\right) M_{23}\left(k_{5}, q\right)-4 m_{1} m_{2}\left(3 \sigma^{2}-1\right) M_{14}\left(k_{5}, q\right) M_{23}\left(p_{1}, q\right)
\end{aligned}
$$




$$
\begin{aligned}
& +4 m_{1}^{2} m_{2}^{2} \sigma\left(2 \sigma^{2}-1\right) M_{14}\left(e^{\mu}, q\right) M_{23}\left(e^{\nu}, q\right) \eta_{\mu \nu}+8 m_{1}^{2} m_{2}^{2} \sigma\left(2 \sigma^{2}-1\right) M_{14}\left(k_{5}, e^{\mu}\right) M_{23}\left(k_{5}, e^{\nu}\right) \eta_{\mu \nu} \\
& +4 q^{2} m_{1} m_{2} \sigma^{2} M_{14}\left(k_{5}, e^{\mu}\right) M_{23}\left(p_{1}, e^{\nu}\right) \eta_{\mu \nu}-4 q^{2} m_{1} m_{2} \sigma^{2} M_{14}\left(p_{2}, e^{\mu}\right) M_{23}\left(k_{5}, e^{\nu}\right) \eta_{\mu \nu} \\
& +2 q^{2} m_{1} m_{2} \sigma^{2} M_{14}\left(e^{\mu}, q\right) M_{23}\left(p_{1}, e^{\nu}\right) \eta_{\mu \nu}-2 q^{2} m_{1} m_{2} \sigma^{2} M_{14}\left(p_{2}, e^{\mu}\right) M_{23}\left(e^{\nu}, q\right) \eta_{\mu \nu} \\
& +4 m_{1}^{2} m_{2}^{2} \sigma\left(2 \sigma^{2}-1\right) M_{14}\left(e^{\mu}, q\right) M_{23}\left(k_{5}, e^{\nu}\right) \eta_{\mu \nu}+4 m_{1}^{2} m_{2}^{2} \sigma\left(2 \sigma^{2}-1\right) M_{14}\left(k_{5}, e^{\mu}\right) M_{23}\left(e^{\nu}, q\right) \eta_{\mu \nu}
\end{aligned}
$$

where $M_{i j}\left(e_{\mu}, a\right)$ is defined in Eq. (4.3). The complete quadruple cut corresponding to Fig. 7(b) and determining the coefficient $d_{\mathrm{B}}$ in Eq. (5.1) is

$$
C_{4}^{(\mathrm{b})}=C_{4}^{(\mathrm{b}), \mathrm{Si}=0}+C_{4}^{(\mathrm{b}), \mathrm{SO}}+C_{4}^{(\mathrm{b}), S_{1} S_{2}}
$$

The quadruple cut $C_{4}^{(\mathrm{b})}$ corresponding to Fig. 7(b) and determining the coefficient $d_{\overline{\mathrm{B}}}$ in Eq. (5.1) is obtained by interchanging the external momenta $p_{2}$ and $p_{3}$ in Eq. (5.13).

\section{Triple cuts}

The triple cuts, which will be used to determine the coefficients $c_{\triangle}$ and $c_{\nabla}$ in Eq. (5.1), are shown in Fig. 8. They may be obtained by sewing together one Compton and two three-point gravitational amplitudes or by imposing an additional cut condition on one of the matter propagators in the two-particle cut (5.6). We follow this second approach:

$$
\left.C_{3}^{(\mathrm{a})} \equiv 2 i p_{2} \cdot k_{5} C_{2}\right|_{p_{2} \cdot k_{5} \rightarrow 0}, \quad C_{3}^{(\mathrm{b})} \equiv-\left.2 i p_{1} \cdot k_{5} C_{2}\right|_{p_{1} \cdot k_{5} \rightarrow 0} .
$$

They are related by the relabeling $\left(m_{1}, m_{2}, p_{1}, p_{2}, p_{3}, p_{4}, k_{5}\right) \leftrightarrow\left(m_{2}, m_{1}, p_{2}, p_{1}, p_{4}, p_{3},-k_{5}\right)$, so we need to evaluate only one of them. Each of the two cuts may be further separated into two parts related by the interchanges $p_{1} \leftrightarrow p_{4}$ and $p_{2} \leftrightarrow p_{3}$, respectively. They correspond to the symmetry of the triangle integrals $I_{\triangle}$ and $I_{\nabla}$. In the following we will not make explicit this separation.

Since the coefficients $c_{\triangle}$ and $c_{\nabla}$ which will be determined from $C_{3}^{(\mathrm{a})}$ and $C_{3}^{(\mathrm{b})}$ multiply integrals whose leading small- $q$ scaling is classical, $I_{\triangle} \sim|q|^{-1} \sim I_{\nabla}$, it suffices to evaluate the triple cuts only to leading order in the classical limit. That is, we are free to use the leading order part of the relations (2.20) between Lorentz generators, polarization, and spin tensors.

For the spin-independent part of $C_{3}^{(\mathrm{a})}$ we find

$$
\begin{aligned}
\frac{C_{3}^{(\mathrm{a}), S_{i}=0}}{64 \pi^{2} G^{2}}= & \frac{i}{q^{2} P_{2}\left(P_{2}+q^{2}\right)} \varepsilon_{1} \cdot \varepsilon_{4} \varepsilon_{2} \cdot \varepsilon_{3}\left\{-4 m_{1}^{2} P_{2}^{2} q^{2}\left(P_{2}^{2}+P_{2} q^{2}+m_{2}^{2}\left(1-6 \sigma^{2}\right) q^{2}\right)+P_{2}^{4} q^{4}-8 m_{1} m_{2} P_{2}^{3} \sigma q^{4}\right. \\
& +16 m_{1}^{3} m_{2} P_{2} \sigma q^{2}\left(P_{2}^{2}+P_{2} q^{2}+m_{2}^{2}\left(1-2 \sigma^{2}\right) q^{2}\right)+2 m_{1}^{4}\left(P_{2}^{4}+2 P_{2}^{3} q^{2}+2 m_{2}^{2} P_{2}\left(1-4 \sigma^{2}\right) q^{4}+2 m_{2}^{4}\left(1-2 \sigma^{2}\right)^{2} q^{4}\right. \\
& \left.\left.+P_{2}^{2} q^{2}\left(m_{2}^{2}\left(2-8 \sigma^{2}\right)+q^{2}\right)\right)\right\}
\end{aligned}
$$

where $P_{2} \equiv-2 p_{2} \cdot k_{5}$. The presence of the two factors in the denominator, related by $p_{2} \leftrightarrow p_{3}$, exposes the presence mentioned above of two distinct terms related by this transformation.

Expressing the result in terms of the covariant spin vector through Eq. (2.18), the terms in $C_{3}^{(\mathrm{a})}$ that are linear in the covariant spin are

$$
\begin{aligned}
\frac{C_{3}^{(\mathrm{a}), \mathrm{SO}}}{64 \pi^{2} G^{2}}= & \frac{2 \varepsilon_{1} \cdot \varepsilon_{4} \varepsilon_{2} \cdot \varepsilon_{3}}{q^{2} P_{2}\left(P_{2}+q^{2}\right)}\left\{S _ { 1 } ( k _ { 5 } , q ) \left(-\left(q ^ { 4 } \left(m_{1}^{3}\left(m_{2}^{3}\left(8 \sigma^{3}-4 \sigma\right)-4 m_{2} P_{2} \sigma\right)+m_{1}^{2}\left(-8 m_{2}^{2} P_{2} \sigma^{2}+m_{2}^{4}\left(4 \sigma^{2}-1\right)+P_{2}^{2}\right)\right.\right.\right.\right. \\
& \left.+2 m_{2} m_{1} P_{2} \sigma\left(P_{2}-2 m_{2}^{2}\right)+m_{2}^{2} P_{2}^{2}\right)-P_{2} q^{2}\left(4 m_{2} m_{1}^{3} \sigma\left(m_{2}^{2}\left(2-4 \sigma^{2}\right)+3 P_{2}\right)\right. \\
& \left.\left.\left.-2 m_{1}^{2} P_{2}\left(m_{2}^{2}\left(1-10 \sigma^{2}\right)+2 P_{2}\right)-8 m_{2} m_{1} P_{2}^{2} \sigma+P_{2}^{3}\right)+m_{1}^{2} P_{2}^{3}\left(3 P_{2}-8 m_{1} m_{2} \sigma\right)\right)\right) \\
& +S_{2}\left(k_{5}, q\right)\left(\left(q ^ { 4 } \left(m_{1}^{4}\left(m_{2}^{2}\left(1-4 \sigma^{2}\right)+P_{2}\right)+4 m_{2} m_{1}^{3} \sigma\left(m_{2}^{2}\left(1-2 \sigma^{2}\right)+2 P_{2}\right)\right.\right.\right. \\
& \left.+m_{1}^{2} P_{2}\left(2 m_{2}^{2}\left(6 \sigma^{2}-1\right)-3 P_{2}\right)-6 m_{2} m_{1} P_{2}^{2} \sigma+P_{2}^{3}\right) \\
& \left.\left.+m_{1}^{2} P_{2} q^{2}\left(m_{1}^{2}\left(m_{2}^{2}\left(2-8 \sigma^{2}\right)+3 P_{2}\right)+12 m_{2} m_{1} P_{2} \sigma-4 P_{2}^{2}\right)+2 m_{1}^{4} P_{2}^{3}\right)\right) \\
& \left.+\left(q^{2}\left(P_{2}-2 m_{1} m_{2} \sigma\right)\left(q^{2}\left(2 m_{1}^{2}\left(-2 m_{2}^{2} \sigma^{2}+m_{2}^{2}+P_{2}\right)+4 m_{2} m_{1} P_{2} \sigma-P_{2}^{2}\right)+2 m_{1}^{2} P_{2}^{2}\right)\right)\left(S_{1}\left(p_{2}, q\right)-S_{2}\left(p_{1}, q\right)\right)\right\}
\end{aligned}
$$


where

$$
S_{i}(a, b) \equiv a_{\mu} b_{\nu} S^{\mu \nu}\left(p_{i}\right) .
$$

Last, the terms in the triple cut which are linear in both $S_{1}$ and $S_{2}$ are

$$
\begin{aligned}
\frac{C_{3}^{(\mathrm{a}), S_{1} S_{2}}}{64 \pi^{2} G^{2}}= & -\frac{i}{4} \frac{\varepsilon_{1} \cdot \varepsilon_{4} \varepsilon_{2} \cdot \varepsilon_{3}}{q^{2} P_{2}\left(P_{2}+q^{2}\right)}\left\{-m_{1}^{2} P_{2}^{3} S_{1}\left(e^{\mu}, e^{\nu}\right) S_{2}\left(e^{\alpha}, e^{\beta}\right) \eta_{\mu \alpha} \eta_{\beta \nu} q^{4}\right. \\
& -16 m_{1}^{2} P_{2}^{2} S_{1}\left(k_{5}, p_{2}\right) S_{2}\left(p_{1}, q\right) q^{2}+2 m_{1}^{2}\left(8 \sigma m_{1} m_{2}-3 P_{2}\right) P_{2}^{2} S_{1}\left(q, e^{\mu}\right) S_{2}\left(q, e^{\nu}\right) \eta_{\mu \nu} q^{2} \\
& -4 m_{1}^{2} P_{2}^{2} S_{1}\left(p_{2}, e^{\mu}\right) S_{2}\left(k_{5}, e^{\nu}\right) \eta_{\mu \nu} q^{4}+8 m_{1}^{2} P_{2}^{2}\left(P_{2}-2 \sigma m_{1} m_{2}\right) S_{1}\left(k_{5}, e^{\mu}\right) S_{2}\left(q, e^{\nu}\right) \eta_{\mu \nu} q^{2} \\
& -16 \sigma m_{1}^{3} m_{2} P_{2}^{2} S_{1}\left(q, e^{\mu}\right) S_{2}\left(k_{5}, e^{\nu}\right) \eta_{\mu \nu} q^{2}-16 m_{1}^{2} P_{2}^{2} S_{1}\left(k_{5}, p_{2}\right) S_{2}\left(k_{5}, q\right) q^{2} \\
& -4 m_{1}^{2}\left(8 \sigma m_{1} m_{2}-5 P_{2}\right) P_{2} S_{1}\left(p_{2}, q\right) S_{2}\left(k_{5}, q\right) q^{2}+16 m_{1}^{2} P_{2}^{2} S_{1}\left(k_{5}, q\right) S_{2}\left(k_{5}, p_{1}\right) q^{2} \\
& +4\left(q^{2}\left(8\left(2 \sigma^{2} m_{2}^{2}+m_{2}^{2}-P_{2}\right) m_{1}^{2}-12 \sigma m_{2} P_{2} m_{1}+3 P_{2}^{2}\right)-8 m_{1}^{2} P_{2}^{2}\right) S_{1}\left(k_{5}, p_{2}\right) S_{2}\left(k_{5}, p_{1}\right) q^{2} \\
& -16 m_{1}^{2} P_{2}^{2} S_{1}\left(p_{2}, q\right) S_{2}\left(k_{5}, p_{1}\right) q^{2}-8 m_{1}^{2} P_{2}^{3} S_{1}\left(q, e^{\mu}\right) S_{2}\left(p_{1}, e^{\nu}\right) \eta_{\mu \nu} q^{2} \\
& +2 P_{2}\left(\left(4\left(3 P_{2}-4 \sigma^{2} m_{2}^{2}\right) m_{1}^{2}+12 \sigma m_{2} P_{2} m_{1}-3 P_{2}^{2}\right) q^{2}+8 m_{1}^{2} P_{2}^{2}\right) S_{1}\left(k_{5}, e^{\mu}\right) S_{2}\left(p_{1}, e^{\nu}\right) \eta_{\mu \nu} q^{2} \\
& -2 P_{2}\left(\left(\left(\left(8-64 \sigma^{2}\right) m_{2}^{2}+20 P_{2}\right) m_{1}^{2}+52 \sigma m_{2} P_{2} m_{1}-11 P_{2}^{2}\right) q^{2}+16 m_{1}^{2} P_{2}^{2}\right) S_{1}\left(k_{5}, q\right) S_{2}\left(p_{1}, q\right) \\
& +2 P_{2}\left(\left(32 \sigma m_{2} m_{1}^{3}-8\left(3 P_{2}-7 \sigma^{2} m_{2}^{2}\right) m_{1}^{2}-42 \sigma m_{2} P_{2} m_{1}+8 P_{2}^{2}\right) q^{2}\right. \\
& \left.+8 m_{1}^{2}\left(4 \sigma m_{1} m_{2}-3 P_{2}\right) P_{2}\right) S_{1}\left(k_{5}, q\right) S_{2}\left(k_{5}, q\right) \\
& +4 m_{1}\left(\left(8 \sigma m_{2}\left(\left(1-2 \sigma^{2}\right) m_{2}^{2}+P_{2}\right) m_{1}^{2}+2 P_{2}\left(\left(6 \sigma^{2}-2\right) m_{2}^{2}+P_{2}\right) m_{1}-3 \sigma m_{2} P_{2}^{2}\right) q^{2}\right. \\
& \left.\left.+8 \sigma m_{1}^{2} m_{2} P_{2}^{2}\right) S_{1}\left(k_{5}, e^{\mu}\right) S_{2}\left(k_{5}, e^{\nu}\right) \eta_{\mu \nu} q^{2}\right\} .
\end{aligned}
$$

The complete triple cut $C_{3}^{(\mathrm{a})}$ in the classical limit is

$$
C_{3}^{(\mathrm{a})}=C_{3}^{(\mathrm{a}), S_{i}=0}+C_{3}^{(\mathrm{a}), \mathrm{SO}}+C_{3}^{(\mathrm{a}), S_{1} S_{2}} .
$$

The triple cut $C_{3}^{(b)}$, corresponding to Fig. 8(b) and determining the coefficient $c_{\nabla}$ in Eq. (5.1), is obtained by applying the transformation $\left(m_{1}, m_{2}, p_{1}, p_{2}, p_{3}, p_{4}, k_{5}\right) \rightarrow$ $\left(m_{2}, m_{1}, p_{2}, p_{1}, p_{4}, p_{3},-k_{5}\right)$ to Eq. (5.19).

\section{Extracting integral coefficients}

Armed with the expressions for the quadruple and triple cuts, we proceed to extract the coefficients of the scalar box and triangle integrals in Eq. (5.1). The construction [31,74] begins with solving the triple and quadruple cut conditions. They determine the loop momentum in terms of one free parameter while the latter, which may be obtained from the former for a special value of that parameter, gives a discrete set of solutions. For a suitable parametrization of the loop momentum, the coefficient of triangle integrals is then obtained as the term in the evaluation of the triple cut on the solution of the cut condition that is independent of the free parameter. The coefficient of the box integrals is given by the sum over the solutions of the quadruple cut conditions of the evaluation of the quadruple cuts on these solutions.

We will begin by solving the triple cut conditions in the appropriate parametrization [31,74]. From here we will extract the loop momentum that solves the quadruple cuts and subsequently use them to extract the integral coefficients. Finally, we reconstruct the classical limit of the oneloop four-point amplitude of arbitrary-spin particles.

\section{The triple cuts and the coefficients of scalar triangle integrals}

Let us consider the triple cut in Fig. 8(a), whose expression is found in Eqs. (5.15)-(5.18). The on-shell conditions for the cut legs are

$$
k_{5}^{2}=0, \quad 2 q \cdot k_{5}-q^{2}=0, \quad p_{1} \cdot k_{5}=0,
$$

where, as before, $q=p_{2}+p_{3}$. Their solution is parametrized as $[31,74]$

$$
k_{5}(T)^{\mu}=x q^{\mu}+y p_{1}^{\mu}+T a_{1}{ }^{\mu}+\frac{\alpha a_{2}{ }^{\mu}}{T},
$$

where $x, y$, and $\alpha$ are free parameters to be determined by (5.20), $T$ parametrizes the component of the loop momentum that is not fixed by the three cut conditions, and the vectors ${a_{1}}^{\mu}$ and $a_{2}{ }^{\mu}$ are given by [74]

$$
a_{1}{ }^{\mu}=\left\langle Q^{b}\left|\sigma^{\mu}\right| P^{b}\right], \quad a_{2}{ }^{\mu}=\left\langle P^{b}\left|\sigma^{\mu}\right| Q^{b}\right] .
$$

The null momenta $P^{b}$ and $Q^{b}$ are chosen to be 


$$
P^{\mathrm{b} \mu} \equiv p_{1}^{\mu}+\frac{m_{1}^{2}}{\gamma} q^{\mu}, \quad Q^{\mathrm{b} \mu} \equiv q^{\mu}+\frac{q^{2}}{\gamma} p_{1}^{\mu} ;
$$

the parameter $\gamma$ is determined by requiring that $P^{\mathrm{b}}$ and $Q^{\mathrm{b}}$ are null,

$$
\gamma=\frac{1}{2}\left(q^{2} \pm \sqrt{q^{2}\left(q^{2}-4 m_{1}^{2}\right)}\right) .
$$

Three of these parameters are fixed by imposing the three on-shell conditions in Eq. (5.20),

$$
\begin{aligned}
& x=-\frac{2 m_{1}^{2}}{q^{2}-4 m_{1}^{2}}, \quad y=-\frac{q^{2}}{q^{2}-4 m_{1}^{2}}, \\
& \alpha=\frac{m_{1}^{2} q^{2}}{2\left(q^{2}-4 m_{1}^{2}\right) a_{1} \cdot a_{2}} .
\end{aligned}
$$

The construction of solutions to the on-shell conditions corresponding to the triple cut in Fig. 8(b) is similar and may be obtained from that corresponding to Fig. 8(a) through

$$
m_{1} \rightarrow m_{2}, \quad y \rightarrow-y, \quad \gamma \rightarrow-\gamma .
$$

Evaluating the triple cut (5.15)-(5.18) on these solutions yields rational functions of the remaining free parameter $T$. The singularities of these functions have different physical interpretations. As discussed before, Eqs. (5.15)-(5.18) contain propagator singularities that correspond to the contributions of the box scalar integrals in Eq. (5.1) to the triple cut. They are reflected by singularities at values of $T$ solving the equations

$$
k_{5}(T) \cdot p_{2}=0 \quad \text { or } \quad k_{5}(T) \cdot p_{3}=0 .
$$

Each of them is a quadratic equation for $T$ and thus has two solutions; it is not difficult to see that, away from special momentum configurations, $T$ takes some finite values. From the discussion above it is clear that the (sum over the) corresponding residues are closely related to the box integral coefficients, which may indeed be extracted this way: the solutions of the first (5.27) equation lead to $d_{\mathrm{B}}$ while those of the second (5.27) equation lead to $d_{\overline{\mathrm{B}}}$.

It has been shown in $[31,74]$ that the coefficient of the triangle integral is given by the $T$-independent part of the average of the evaluation of the triple cut (5.15)-(5.18) on the two solutions of the triple-cut on-shell conditions, Eqs. (5.21), (5.22), (5.25), and (5.24). The relevant terms come therefore from the $T$-independent parts of the loop momentum $k_{5}(T)$ as well as from terms containing the product $a_{1}^{\mu} a_{2}^{\nu}$. This product is given by

$$
a_{1}^{\mu} a_{2}^{\nu}=2\left(Q^{b \mu} P^{b \nu}+Q^{b \nu} P^{b \mu}-\eta^{\mu \nu} Q^{b} \cdot P^{b}\right)-2 i \epsilon^{\mu \nu \alpha \beta} Q_{\alpha}^{b} P_{\beta}^{b},
$$

which can be expressed in them of the full external momenta using Eq. (5.23).

Following this procedure, the triple cut $C_{3}^{(\mathrm{a})}$, corresponding to Fig. 8(a), yields the coefficient of the triangle integral $I_{\triangle}$ in Eq. (5.1):

$$
\begin{aligned}
c_{\triangle}= & -32 \pi^{2} G^{2} m_{1}^{2} \varepsilon_{1} \cdot \varepsilon_{4} \varepsilon_{2} \cdot \varepsilon_{3}\left\{6 m_{1}^{2} m_{2}^{2}\left(5 \sigma^{2}-1\right)+\frac{2 m_{1} m_{2}\left(5 \sigma^{2}-3\right) \sigma}{\sigma^{2}-1}\left(3 i S_{2}\left(p_{1}, q\right)-4 i S_{1}\left(p_{2}, q\right)\right)\right. \\
& +\frac{2}{\left(\sigma^{2}-1\right)}\left\{3\left(5 \sigma^{2}-1\right) S_{1}\left(p_{2}, q\right) S_{2}\left(p_{1}, q\right)+\left(5 \sigma^{2}-3\right) \sigma \eta_{\mu \alpha}\left[m _ { 1 } m _ { 2 } \left(S_{1}\left(e^{\mu}, q\right) S_{2}\left(e^{\alpha}, q\right)\right.\right.\right. \\
& \left.-q^{2} \eta_{\nu \beta} S_{1}\left(e^{\mu}, e^{\nu}\right) S_{2}\left(e^{\alpha}, e^{\beta}\right)\right)+\frac{q^{2}}{\sigma^{2}-1}\left(2 \sigma S_{1}\left(e^{\mu}, p_{2}\right) S_{2}\left(e^{\alpha}, p_{1}\right)\right. \\
& \left.\left.\left.\left.+\frac{m_{1}+m_{2} \sigma}{m_{2}} S_{1}\left(e^{\mu}, p_{2}\right) S_{2}\left(e^{\alpha}, q\right)-\frac{m_{2}+m_{1} \sigma}{m_{1}} S_{2}\left(e^{\mu}, p_{1}\right) S_{1}\left(e^{\alpha}, q\right)\right)\right]\right\}\right\}+\mathcal{O}\left(q^{2} S_{i}^{2}\right) .
\end{aligned}
$$

The coefficient $c_{\nabla}$, of the integral $I_{\nabla}$, can be obtained from $c_{\triangle}$ through the map

$$
\begin{aligned}
& \left(m_{1}, m_{2}, p_{1}, p_{2}, S_{1}, S_{2}, q, \sigma\right) \\
& \quad \rightarrow\left(m_{2}, m_{1}, p_{2}, p_{1}, S_{2}, S_{1},-q, \sigma\right) .
\end{aligned}
$$

\section{The quadruple cuts and the coefficients of scalar box integrals}

The coefficients of scalar box integrals are given by the average over the values of the quadruple cut on the solutions of the quadruple cut conditions [27,31,74]. As discussed in the previous subsection, these solutions may be obtained from those of the triple cuts, Eqs. (5.21), (5.22), (5.25), and (5.24), by further demanding that the additional propagator of the desired box diagram is on shell. We may, alternatively, start with a parametrization of the loop momentum which is slightly more convenient for this purpose,

$$
k_{5}^{\mu}=\alpha p_{1}^{\mu}+\beta p_{2}^{\mu}+\gamma q^{\mu}+\delta \eta^{\mu}
$$


where $\alpha, \beta, \gamma$, and $\delta$ are free, complex parameters and $\eta$ is a null reference vector, whose precise value should not affect the final answer. By choosing $\eta$ to be orthogonal to $p_{1}$ and $p_{2}$, we find the two solutions for the loop momentum

$k_{5}^{\mu}=\frac{q^{2}}{2 q \cdot \eta} \eta^{\mu}, \quad k_{5}^{\mu}=\frac{N_{\alpha} p_{1}^{\mu}+N_{\beta} p_{2}^{\mu}+N_{\gamma} q^{\mu}}{\mathcal{N}}-\frac{q^{2}}{2 q \cdot \eta} \eta^{\mu}$,

where

$$
N_{\alpha}=-2 m_{2}\left(m_{2}+m_{1} \sigma\right) q^{2}, \quad N_{\beta}=2 m_{1}\left(m_{1}+m_{2} \sigma\right) q^{2},
$$

$$
N_{\gamma}=4 m_{1}^{2} m_{2}^{2}\left(\sigma^{2}-1\right), \quad \mathcal{N}=N_{\gamma}+\left(N_{\beta}-N_{\alpha}\right) / 2 .
$$

While it is possible to keep $\eta$ arbitrary (up to its properties stated above) and have it drop out of the final expressions for $d_{\mathrm{B}}$, it is more convenient to use an explicit form that manifests its properties. We choose it to be

$$
\begin{aligned}
& \eta^{\mu}=\left\langle p_{1}^{\mathrm{b}}\left|\sigma^{\mu}\right| p_{2}^{\mathrm{b}}\right], \quad p_{1}^{\mathrm{b}}=p_{1}+m_{1}^{2} \zeta p_{2}, \\
& p_{2}^{\mathrm{b}}=p_{2}+m_{2}^{2} \zeta p_{1}, \quad \zeta=-\frac{\sigma \pm \sqrt{\sigma^{2}-1}}{m_{1} m_{2}} .
\end{aligned}
$$

The two values of $\zeta$ are determined by demanding that $p_{1}^{b}$ and $p_{2}^{b}$ are null. Both are necessary for determining the coefficient of the box integral. To express the dependence on $\eta$ in terms of $p_{1}$ and $p_{2}$ it is useful to multiply and divide the $\eta$-dependent terms in Eq. (5.32) by $q \cdot \bar{\eta}$,

$$
\frac{\eta^{\mu}}{q \cdot \eta}=\frac{q \cdot \bar{\eta} \eta^{\mu}}{q \cdot \bar{\eta} q \cdot \eta}
$$

and use the identity

$$
\begin{aligned}
\eta^{\mu} \bar{\eta}^{\nu}= & 2\left(p_{1}{ }^{b \mu} p_{2}{ }^{b \nu}+p_{2}{ }^{b \mu} p_{1}{ }^{b \nu}-\eta^{\mu \nu} p_{1}{ }^{b} \cdot p_{2}{ }^{b}\right) \\
& -2 i \epsilon^{\mu \nu \alpha \beta} p_{1}{ }^{b}{ }_{\alpha} p_{2}{ }^{b} .
\end{aligned}
$$

Using this procedure on the quadruple cut Eqs. (5.13), we find that the coefficient $d_{\mathrm{B}}$ of the box integral $I_{\mathrm{B}}$ is given by

$$
\begin{aligned}
d_{\mathrm{B}}= & 64 G^{2} m_{1} m_{2} \pi^{2}\left\{4 m_{1}^{3} m_{2}^{3}\left(2 \sigma^{2}-1\right)^{2} \varepsilon_{1} \cdot \varepsilon_{4} \varepsilon_{2} \cdot \varepsilon_{3}+8 i m_{1}^{2} m_{2}^{2} \sigma\left(2 \sigma^{2}-1\right)\left(\varepsilon_{1} \cdot \varepsilon_{4} M_{23}\left(p_{1}, q\right)\right.\right. \\
& \left.-M_{14}\left(p_{2}, q\right) \varepsilon_{2} \cdot \varepsilon_{3}\right)+4 m_{1} m_{2}\left(2 \sigma^{2}-1\right) M_{14}\left(p_{2}, q\right) M_{23}\left(p_{1}, q\right) \\
& +4 m_{1}^{2} m_{2}^{2} \sigma\left(2 \sigma^{2}-1\right) \eta_{\mu \nu} M_{14}\left(e^{\mu}, q\right) M_{23}\left(e^{\nu}, q\right)-2 q^{2} m_{1}^{2} m_{2}^{2} \sigma\left(2 \sigma^{2}-1\right) \eta_{\mu \nu} \eta_{\rho \sigma} M_{14}\left(e^{\mu}, e^{\rho}\right) M_{23}\left(e^{\nu}, e^{\sigma}\right) \\
& +\frac{2 q^{2} m_{1} m_{2}}{\sigma^{2}-1}\left(4 \sigma^{4}-2 \sigma^{2}-1\right) \eta_{\mu \nu} M_{14}\left(e^{\mu}, p_{2}\right) M_{23}\left(e^{\nu}, p_{1}\right) \\
& -\frac{q^{2} m_{2}}{\sigma^{2}-1}\left(\left(4 \sigma^{4}-2 \sigma^{2}-1\right) m_{1}+\sigma\left(4 \sigma^{2}-3\right) m_{2}\right) \eta_{\mu \nu} M_{14}\left(e^{\mu}, q\right) M_{23}\left(e^{\nu}, p_{1}\right) \\
& \left.+\frac{q^{2} m_{1}}{\sigma^{2}-1}\left(\left(4 \sigma^{4}-2 \sigma^{2}-1\right) m_{2}+\sigma\left(4 \sigma^{2}-3\right) m_{1}\right) \eta_{\mu \nu} M_{14}\left(e^{\mu}, p_{2}\right) M_{23}\left(e^{\nu}, q\right)\right\}+\mathcal{O}\left(q^{3}\right),
\end{aligned}
$$

where $M_{i j}(a, b)$ and their counterparts with free indices are defined in Eqs. (4.3). As in the case of the quadruple cut, we kept intact the dependence on Lorentz generators and polarization tensors, anticipating that comparison with the effective field theory will require a careful choice of the subleading in the classical limit.

The coefficient $d_{\overline{\mathrm{B}}}$ of the second crossed-box integral $I_{\overline{\mathrm{B}}}$ is obtained from $d_{\mathrm{B}}$ above by interchanging $p_{2}$ and $p_{3}$.

\section{The one-loop amplitude in the classical limit}

We can now reconstruct the classical part of the fourpoint amplitude (5.1). As we will see in detail in the next section and used in earlier literature [11,12,22,23, $33,36,37]$, the new physical information in this amplitude arises from triangle integrals. It is therefore convenient to collect their contribution in $\mathcal{M}_{\triangle+\nabla}$ defined as

$$
\begin{aligned}
i \mathcal{M}_{4}^{1 \text { loop }} & =d_{\mathrm{B}} I_{\mathrm{B}}+d_{\overline{\mathrm{B}}} I_{\overline{\mathrm{B}}}+c_{\triangle} I_{\triangle}+c_{\nabla} I_{\nabla} \\
& \equiv d_{\mathrm{B}} I_{\mathrm{B}}+d_{\overline{\mathrm{B}}} I_{\overline{\mathrm{B}}}+\mathcal{M}_{\triangle+\nabla} .
\end{aligned}
$$

The two triangle integrals are related by interchanging the masses $m_{1}$ and $m_{2}$ and are well-known; in an expansion around the classical limit they are $[11,12,17,33]$ 


$$
\begin{aligned}
& I_{\triangle}=\int \frac{d^{4} l}{(2 \pi)^{2}} \frac{1}{\left(l^{2}+i \epsilon\right)\left((l+q)^{2}+i \epsilon\right)\left(\left(l+p_{1}\right)^{2}-m_{1}^{2}+i \epsilon\right)}=-\frac{i}{32 m_{1}} \frac{1}{\sqrt{-q^{2}}}+\cdots, \\
& I_{\nabla}=\int \frac{d^{4} l}{(2 \pi)^{2}} \frac{1}{\left(l^{2}+i \epsilon\right)\left((l+q)^{2}+i \epsilon\right)\left(\left(l-p_{2}\right)^{2}-m_{2}^{2}+i \epsilon\right)}=-\frac{i}{32 m_{2}} \frac{1}{\sqrt{-q^{2}}}+\cdots .
\end{aligned}
$$

Thus, together with their coefficients (5.29), their contribution to the amplitude is

$$
\begin{aligned}
i \mathcal{M}_{\triangle+\nabla}= & \frac{2 \pi^{2} i G^{2} m_{1} m_{2}}{\sqrt{-q^{2}}} \varepsilon_{1} \cdot \varepsilon_{4} \varepsilon_{2} \cdot \varepsilon_{3}\left\{3 m_{1} m_{2}\left(m_{1}+m_{2}\right)\left(5 \sigma^{2}-1\right)\right. \\
& +\frac{\left(5 \sigma^{2}-3\right) \sigma}{\sigma^{2}-1}\left(\left(3 m_{1}+4 m_{2}\right) i S_{2}\left(p_{1}, q\right)-\left(3 m_{2}+4 m_{1}\right) i S_{1}\left(p_{2}, q\right)\right) \\
& +\frac{\left(m_{1}+m_{2}\right)}{\left(\sigma^{2}-1\right) m_{1} m_{2}}\left\{3\left(5 \sigma^{2}-1\right) S_{1}\left(p_{2}, q\right) S_{2}\left(p_{1}, q\right)+\left(5 \sigma^{2}-3\right) \sigma \eta_{\mu \alpha}\left[m _ { 1 } m _ { 2 } \left(S_{1}\left(e^{\mu}, q\right) S_{2}\left(e^{\alpha}, q\right)\right.\right.\right. \\
& \left.-q^{2} \eta_{\nu \beta} S_{1}\left(e^{\mu}, e^{\nu}\right) S_{2}\left(e^{\alpha}, e^{\beta}\right)\right)+\frac{q^{2}}{\sigma^{2}-1}\left(2 \sigma S_{1}\left(e^{\mu}, p_{2}\right) S_{2}\left(e^{\alpha}, p_{1}\right)\right. \\
& \left.\left.\left.\left.+\frac{m_{1}+m_{2} \sigma}{m_{2}} S_{1}\left(e^{\mu}, p_{2}\right) S_{2}\left(e^{\alpha}, q\right)-\frac{m_{2}+m_{1} \sigma}{m_{1}} S_{2}\left(e^{\mu}, p_{1}\right) S_{1}\left(e^{\alpha}, q\right)\right)\right]\right\}\right\}+\mathcal{O}\left(q^{2} S_{i}^{2}\right) .
\end{aligned}
$$

Using identities of the Levi-Civita tensor, this can be expressed in terms of the covariant spin vector:

$$
\begin{aligned}
i \mathcal{M}_{\triangle+\nabla}= & \frac{\pi^{2} i G^{2} m_{1} m_{2}}{\sqrt{-q^{2}}} \varepsilon_{1} \cdot \varepsilon_{4} \varepsilon_{2} \cdot \varepsilon_{3}\left\{6 m_{1} m_{2}\left(m_{1}+m_{2}\right)\left(5 \sigma^{2}-1\right)\right. \\
& +\frac{2 i\left(5 \sigma^{2}-3\right) \sigma}{\sigma^{2}-1} \epsilon^{\mu \nu \rho \sigma} p_{1 \mu} p_{2 \nu} q_{\rho}\left(\left(3 m_{1}+4 m_{2}\right) \frac{S_{2 \sigma}}{m_{2}}+\left(3 m_{2}+4 m_{1}\right) \frac{S_{1 \sigma}}{m_{1}}\right) \\
& +\frac{2\left(m_{1}+m_{2}\right)\left(20 \sigma^{4}-21 \sigma^{2}+3\right)}{\sigma^{2}-1}\left(q \cdot S_{1} q \cdot S_{2}-q^{2} S_{1} \cdot S_{2}\right) \\
& \left.+\frac{8 q^{2} \sigma^{3}\left(m_{1}+m_{2}\right)\left(5 \sigma^{2}-4\right) p_{1} \cdot S_{2} p_{2} \cdot S_{1}}{m_{1} m_{2}\left(\sigma^{2}-1\right)^{2}}\right\}+\mathcal{O}\left(q^{2} S_{i}^{2}\right)
\end{aligned}
$$

The terms containing both $S_{1}$ and $S_{2}$ are now expressed as scalar products; this property will be useful in Sec. VI for systematically organizing the interactions of spinning particles. The box integrals $I_{\mathrm{B}}$ and $I_{\overline{\mathrm{B}}}$ expanded in the classical limit are also well-known [11,17,32]; we do not list explicitly their contribution to the complete amplitude (5.40) because, on the one hand, it will turn out to be physically unimportant and, on the other, it can easily be reconstructed given the $d_{\mathrm{B}}$ integral coefficient in Eq. (5.38). Later in this section we compare Eq. (5.40) with existing results in the literature $[34,60,66]$.

The complete classical amplitude (5.40) agrees with the spin-1/2 amplitude constructed in [66] after changing the orientation of external momenta in the latter to match ours and upon making the replacements

$\varepsilon_{i} \cdot \varepsilon_{j} \rightarrow 1, \quad \varepsilon_{1} M^{\mu \nu} \varepsilon_{4}=S^{\mu \nu}\left(p_{1}\right), \quad \varepsilon_{2} M^{\mu \nu} \varepsilon_{3}=S^{\mu \nu}\left(p_{2}\right)$.
The former can be understood as a choice of normalization of the amplitude, and the latter two are consistent with the leading term in our second Eq. (2.20) and are a consequence of the effective spinors used in [66].

References [34,60] present the amplitude as different expansions around the zero-momentum limit: in the former it is an expansion in the spatial momenta of external particles while in the latter it is an expansion in $(\sigma-1)$. Accounting for the nonrelativistic normalization of [34] it is not difficult to see that the small momentum expansion of our expression for $\mathcal{M}_{\triangle+\nabla}$ recovers the terms listed in Eq. (94) of that reference and the expansion in $(\sigma-1)$ of $\mathcal{M}_{\triangle+\nabla}$ recovers the terms listed in Eqs. (7.11), (7.13), and (7.18) of Ref. [60].

\section{E. Tree and one-loop summary}

To facilitate the extraction of the two-body effective Hamiltonian in the next section, we now summarize the one-loop and tree-level four-higher-spin amplitudes obtained in this section and Sec. IV, respectively. For this 
purpose we normalize the amplitudes nonrelativistically, by dividing by a factor of $4 E_{1} E_{2}$, and manifest the dependence on the rest-frame spin that arises from the presence of Lorentz generators in vertices. We do the latter in two steps: we will first expose the rest-frame spins coming from the dependence on the covariant spin vectors in the amplitude, but not that coming from the product of polarization tensors. The coefficients of the various spin-dependent monomials in the resulting expressions are decorated with a subscript "cov," which emphasizes their covariant origin. We subsequently extract the remaining spin dependence in the product of polarization tensors. The reason for this stepwise treatment is that the coefficients of the various spin-dependent monomials in the final expressions are simple linear combinations of the "covariant" coefficients, with additional energy- and mass-dependent factors arising from Eqs. (2.20) and (3.10).

To further facilitate comparison with EFT calculations in the next section, we specialize the expressions of the amplitudes and their associated particle spins to the center-of-mass frame, defined as (recall that all momenta are outgoing)

$$
\begin{array}{ll}
p_{1}=-\left(E_{1}, \boldsymbol{p}\right), & p_{2}=-\left(E_{2},-\boldsymbol{p}\right), \\
q=(0, \boldsymbol{q}), & \boldsymbol{p} \cdot \boldsymbol{q}=\frac{\boldsymbol{q}^{2}}{2} .
\end{array}
$$

Equation (2.17) then gives the relation between the covariant spin vectors and the corresponding rest-frame ones:

$$
\begin{aligned}
& S_{1}^{\mu}=\left(\frac{\boldsymbol{p} \cdot \boldsymbol{S}_{1}}{m_{1}}, \boldsymbol{S}_{1}+\frac{\boldsymbol{p} \cdot \boldsymbol{S}_{1}}{\left(E_{1}+m_{1}\right) m_{1}} \boldsymbol{p}\right), \\
& S_{2}^{\mu}=\left(-\frac{\boldsymbol{p} \cdot \boldsymbol{S}_{2}}{m_{2}}, \boldsymbol{S}_{2}+\frac{\boldsymbol{p} \cdot \boldsymbol{S}_{2}}{\left(E_{2}+m_{2}\right) m_{2}} \boldsymbol{p}\right) .
\end{aligned}
$$

The two covariant spin-dependent factors in the treelevel amplitude Eq. (4.8) are $q \cdot S_{i}$ and $p_{1 \mu} p_{2 \nu} q_{\rho} S_{i \sigma}$. Using Eqs. (5.46), it is straightforward to find that

$$
\begin{aligned}
q \cdot S_{i} & =\boldsymbol{q} \cdot \boldsymbol{S}_{i}-\frac{\boldsymbol{q}^{2} \boldsymbol{p} \cdot \boldsymbol{S}_{i}}{2 m_{i}\left(E_{i}+m_{i}\right)}, \\
\epsilon^{\mu \nu \rho \sigma} p_{1 \mu} p_{2 \nu} q_{\rho} S_{i \sigma} & =E(\boldsymbol{p} \times \boldsymbol{q}) \cdot \boldsymbol{S}_{i},
\end{aligned}
$$

where $E=E_{1}+E_{2}$ is the total energy of the incoming particles and we neglected terms that are of a higher-order in $\boldsymbol{q}$, which appear because of the fourth relation in (5.45). While such terms are important at loop-level, they can be safely ignored at tree level because they cancel the graviton propagator and thus cannot contribute to the long-range potential.

With these preliminaries, the tree-level (4.8) with a nonrelativistic normalization can be written as

$$
\begin{aligned}
\frac{\mathcal{M}_{4}^{\text {tree }}}{4 E_{1} E_{2}}= & \frac{4 \pi G}{\boldsymbol{q}^{2}} \varepsilon_{4} \cdot \varepsilon_{1} \varepsilon_{3} \cdot \varepsilon_{2}\left[a_{\mathrm{cov}, 1}^{(0)}+i \sum_{j=1}^{2} a_{\mathrm{cov}, 1}^{(1, j)}(\boldsymbol{p} \times \boldsymbol{q}) \cdot \boldsymbol{S}_{j}\right. \\
& \left.+a_{\mathrm{cov}, 1}^{(2,1)} \boldsymbol{q} \cdot \boldsymbol{S}_{1} \boldsymbol{q} \cdot \boldsymbol{S}_{2}\right]
\end{aligned}
$$

The cov subscript decorating the coefficients reflects the fact that they originate from terms with covariant dependence on the spin vector; the second subscript "1" reflects that they are tree-level coefficients. The first superscript in the $a$ coefficients represents the number of spin vectors multiplying this coefficient while the second superscript denotes the spin-dependent monomials with the given number of spins. For monomials linear in spin we identify it with the spin label. While here we encounter a single twospin monomial, we chose to nevertheless index it in anticipation of the fact that more monomials will appear in the one-loop amplitude. The explicit expressions for the coefficients can easily be read off from the amplitude (4.8); accounting for the nonrelativistic normalization, they are

$\begin{array}{ll}a_{\mathrm{cov}, 1}^{(0)} & =-\frac{m^{2} \nu^{2}}{\xi \gamma^{2}}\left(1-2 \sigma^{2}\right), \\ a_{\mathrm{cov}, 1}^{(2,1)} & =-\frac{\nu}{\xi \gamma^{2}}\left(1-2 \sigma^{2}\right),\end{array}$

where we used the variables

$$
\begin{aligned}
& m=m_{1}+m_{2}, \quad E=E_{1}+E_{2}, \quad \gamma=\frac{E}{m}, \\
& \nu=\frac{m_{1} m_{2}}{m^{2}}, \quad \xi=\frac{E_{1} E_{2}}{E^{2}} .
\end{aligned}
$$

It is interesting to note that a second two-spin monomial appears, $\boldsymbol{q}^{2} \boldsymbol{S}_{1} \cdot \boldsymbol{S}_{2}$, with coefficient equal to $a_{\mathrm{cov}, 1}^{(2,2)}=-a_{\mathrm{cov}, 1}^{(2,1)}$. Because of the factor of $\boldsymbol{q}^{2}$, however, it cannot contribute to a long-range interaction at tree level, so it is dropped. This structure will appear again at one loop, where the factor of $q^{2}$ no longer implies that this monomial can be dropped.

The one-loop amplitude is given in Eqs. (5.40), (5.38), and (5.43); it contains two additional covariant spindependent monomials, $p_{2} \cdot S_{1}$ and $p_{1} \cdot S_{2}$, apart from those already appearing at tree level. They can easily be expressed in terms of the rest-frame spin vectors,

$p_{2} \cdot S_{1}=-\frac{E}{m_{1}} \boldsymbol{p} \cdot \boldsymbol{S}_{1}, \quad p_{1} \cdot S_{2}=+\frac{E}{m_{2}} \boldsymbol{p} \cdot \boldsymbol{S}_{2}$,

using Eq. (5.46). Together with Eqs. (5.47) they can be used to write the one-loop amplitude as 


$$
\begin{aligned}
\frac{\mathcal{M}_{4}^{1 \text { loop }}}{4 E_{1} E_{2}}= & \frac{2 \pi^{2} G^{2}}{|\boldsymbol{q}|} \varepsilon_{4} \cdot \varepsilon_{1} \varepsilon_{3} \cdot \varepsilon_{2}\left[a_{\mathrm{cov}, 2}^{(0)}+i \sum_{j=1}^{2} a_{\mathrm{cov}, 2}^{(1, j)}(\boldsymbol{p} \times \boldsymbol{q}) \cdot \boldsymbol{S}_{j}+a_{\mathrm{cov}, 2}^{(2,1)} \boldsymbol{q} \cdot \boldsymbol{S}_{1} \boldsymbol{q} \cdot \boldsymbol{S}_{2}+a_{\mathrm{cov}, 2}^{(2,2)} \boldsymbol{q}^{2} \boldsymbol{S}_{1} \cdot \boldsymbol{S}_{2}+a_{\mathrm{cov}, 2}^{(2,3)} \boldsymbol{q}^{2} \boldsymbol{p} \cdot \boldsymbol{S}_{1} \boldsymbol{p} \cdot \boldsymbol{S}_{2}\right] \\
& -i a_{\mathrm{B}} I_{\mathrm{B}}-i a_{\overline{\mathrm{B}}} I_{\overline{\mathrm{B}}} .
\end{aligned}
$$

For the terms on the first two lines we used the same labeling scheme for the coefficients as at tree level. The two subscripts indicate that the coefficients originate from covariant dependence on the spin vectors and that they appear at one-loop, respectively. The new superscripts compared to those already appearing for tree-level coefficients indicate that they multiply bilinears in spin which are labeled as 2 and 3, continuing the list of monomials bilinears in spin started at tree level. They are

$$
\begin{array}{ll}
a_{\mathrm{cov}, 2}^{(0)}=\frac{3 \nu^{2} m^{3}}{4 \xi \gamma^{2}}\left(5 \sigma^{2}-1\right), & \\
a_{\mathrm{cov}, 2}^{(1,1)}=\frac{\nu}{4 \xi \gamma^{2}} \frac{\sigma\left(5 \sigma^{2}-3\right)}{\sigma^{2}-1} \frac{\left(4 m_{1}+3 m_{2}\right)}{m_{1}} E, \quad a_{\mathrm{cov}, 2}^{(1,2)}=\frac{\nu}{4 \xi \gamma^{2}} \frac{\sigma\left(5 \sigma^{2}-3\right)}{\sigma^{2}-1} \frac{\left(3 m_{1}+4 m_{2}\right)}{m_{2}} E, \\
a_{\mathrm{cov}, 2}^{(2,1)}=-a_{\mathrm{cov}, 2}^{(2,2)}=\frac{m \nu}{4 \xi \gamma^{2}}\left(\frac{20 \sigma^{4}-21 \sigma^{2}+3}{\sigma^{2}-1}\right), \quad a_{\mathrm{cov}, 2}^{(2,3)}=\frac{m \nu}{4 \xi \gamma^{2}}\left(\frac{20 \sigma^{3}-15 \sigma^{2}-6 \sigma+3}{(\sigma-1) \boldsymbol{p}^{2}}\right) .
\end{array}
$$

We point out here the appearance, as in the tree-level amplitude, of the monomial $\boldsymbol{q}^{2} \boldsymbol{S}_{1} \cdot \boldsymbol{S}_{2}$ with coefficient $a_{\mathrm{cov}, 2}^{(2,2)}=-a_{\mathrm{cov}, 2}^{(2,1)}$. It would be interesting to understand whether this equality persists to higher orders in perturbation theory. The box integral coefficients,

$$
a_{\mathrm{B}}=\frac{d_{\mathrm{B}}}{4 E^{2} \gamma_{1} \gamma_{2}}, \quad a_{\overline{\mathrm{B}}}=\frac{d_{\overline{\mathrm{B}}}}{4 E^{2} \gamma_{1} \gamma_{2}},
$$

where $\gamma_{i}=E_{i} / m_{i}$ is the usual Lorentz factor of particle $i$. The corresponding box and cross-box integrals, $I_{\mathrm{B}}$ and $I_{\overline{\mathrm{B}}}$, are given in Ref. [89] and were evaluated in the classical limit in Ref. [12]. In Sec. VI we follow the same integration scheme where the integrals are

$$
\begin{aligned}
I_{\mathrm{B}} & =\frac{i}{2 E} \int \frac{d^{D-1} \boldsymbol{\ell}}{(2 \pi)^{D-1}} \frac{1}{\boldsymbol{\ell}^{2}(\boldsymbol{\ell}+\boldsymbol{q})^{2}\left(\boldsymbol{\ell}^{2}+2 \boldsymbol{p} \boldsymbol{\ell}\right)}, \\
I_{\overline{\mathrm{B}}} & =0 .
\end{aligned}
$$

The box integral has a stronger-than-classical scaling in the classical limit. Thus, in taking the classical limit of the box coefficient $d_{\mathrm{B}}$, first subleading terms should also be kept. As we will discuss in the next section, they have no physical effects, and we will choose them such that the EFT we construct there corresponds to the complete theory used to compute the amplitudes summarized here. For this reason we did not express the box (and cross-box) coefficients in terms of the rest-frame spin vectors; the leading terms are, however, easy to obtain by replacing Eqs. (5.47) and (5.51) in Eq. (5.38).

It is not difficult to see that, upon expanding the products of polarization tensors in Eqs. (5.48) and (5.52) using Eqs. (2.20), no further spin-dependent spin-bilinear monomials are generated. The coefficients of each spin-dependent monomial is slightly modified because of the additional spin dependence coming from $\varepsilon_{4} \cdot \varepsilon_{1} \varepsilon_{3} \cdot \varepsilon_{2}$ : each one becomes a linear combination of $a_{\mathrm{cov}}$. This mixing is the same at every order in $G$. Thus, the tree-level and one-loop amplitudes fully expanded to second order in spin are

$$
\begin{gathered}
\frac{\mathcal{M}_{4}^{\text {tree }}}{4 E_{1} E_{2}}=\frac{4 \pi G}{\boldsymbol{q}^{2}}\left[a_{1}^{(0)}+i \sum_{j=1}^{2} a_{1}^{(1, j)}(\boldsymbol{p} \times \boldsymbol{q}) \cdot \boldsymbol{S}_{j}+a_{1}^{(2,1)} \boldsymbol{q} \cdot \boldsymbol{S}_{1} \boldsymbol{q} \cdot \boldsymbol{S}_{2}\right] \\
\frac{\mathcal{M}_{4}^{1 \text { loop }}}{4 E_{1} E_{2}}=\frac{2 \pi^{2} G^{2}}{|\boldsymbol{q}|}\left[a_{2}^{(0)}+i \sum_{j=1}^{2} a_{2}^{(1, j)}(\boldsymbol{p} \times \boldsymbol{q}) \cdot \boldsymbol{S}_{j}+a_{2}^{(2,1)} \boldsymbol{q} \cdot \boldsymbol{S}_{1} \boldsymbol{q} \cdot \boldsymbol{S}_{2}+a_{2}^{(2,2)} \boldsymbol{q}^{2} \boldsymbol{S}_{1} \cdot \boldsymbol{S}_{2}+a_{2}^{(2,3)} \boldsymbol{q}^{2} \boldsymbol{p} \cdot \boldsymbol{S}_{1} \boldsymbol{p} \cdot \boldsymbol{S}_{2}\right]-i a_{\mathrm{B}} I_{\mathrm{B}}-i a_{\overline{\mathrm{B}}} I_{\overline{\mathrm{B}}}
\end{gathered}
$$


with the $a_{i}^{A}$ given by

$$
\begin{aligned}
a_{i}^{(0)} & =a_{\mathrm{cov}, i}^{(0)}, \\
a_{i}^{(1,1)} & =a_{\mathrm{cov}, i}^{(1,1)}-\frac{1}{m_{1}^{2}\left(\gamma_{1}+1\right)} a_{\mathrm{cov}, i}^{(0)}, \\
a_{i}^{(1,2)} & =a_{\mathrm{cov}, i}^{(1,2)}-\frac{1}{m_{2}^{2}\left(\gamma_{2}+1\right)} a_{\mathrm{cov}, i}^{(0)}, \\
a_{i}^{(2,1)} & =a_{\mathrm{cov}, i}^{(2,1)}-\frac{\boldsymbol{p}^{2}}{m_{2}^{2}\left(\gamma_{2}+1\right)} a_{\mathrm{cov}, i}^{(1,1)}-\frac{\boldsymbol{p}^{2}}{m_{1}^{2}\left(\gamma_{1}+1\right)} a_{\mathrm{cov}, i}^{(1,2)}+\frac{\boldsymbol{p}^{2}}{m_{1}^{2} m_{2}^{2}\left(\gamma_{1}+1\right)\left(\gamma_{2}+1\right)} a_{\mathrm{cov}, i}^{(0)}, \\
a_{i}^{(2,2)} & =a_{\mathrm{cov}, i}^{(2,2)}+\frac{\boldsymbol{p}^{2}}{m_{2}^{2}\left(\gamma_{2}+1\right)} a_{\mathrm{cov}, i}^{(1,1)}+\frac{\boldsymbol{p}^{2}}{m_{1}^{2}\left(\gamma_{1}+1\right)} a_{\mathrm{cov}, i}^{(1,2)}-\frac{\boldsymbol{p}^{2}}{m_{1}^{2} m_{2}^{2}\left(\gamma_{1}+1\right)\left(\gamma_{2}+1\right)} a_{\mathrm{cov}, i}^{(0)}, \\
a_{i}^{(2,3)} & =a_{\mathrm{cov}, i}^{(2,3)}-\frac{1}{m_{2}^{2}\left(\gamma_{2}+1\right)} a_{\mathrm{cov}, i}^{(1,1)}-\frac{1}{m_{1}^{2}\left(\gamma_{1}+1\right)} a_{\mathrm{cov}, i}^{(1,2)}+\frac{1}{m_{1}^{2} m_{2}^{2}\left(\gamma_{1}+1\right)\left(\gamma_{2}+1\right)} a_{\mathrm{cov}, i}^{(0)},
\end{aligned}
$$

with $i=1,2$. We are not decorating them with a cov index because they are no longer associated with a definite combination of covariant spin vectors. We note that, while the expansion of $\varepsilon_{4} \cdot \varepsilon_{1} \varepsilon_{3} \cdot \varepsilon_{2}$ generates the structures $\boldsymbol{q}^{2} \boldsymbol{S}_{1}$. $\boldsymbol{S}_{2}$ and $\boldsymbol{q}^{2} \boldsymbol{p} \cdot \boldsymbol{S}_{1} \boldsymbol{p} \cdot \boldsymbol{S}_{2}$ in the tree-level amplitude, they can be ignored, as done before, because they do not correspond to long-range interaction terms. The $a_{\mathrm{cov}, i}$ with $i=1,2$ are given in Eqs. (5.49) and (5.53), respectively, and $a_{\mathrm{cov}, 1}^{(2,3)}=0$.

In the following section we will use these expressions to fix the effective interaction Hamiltonian of two spinning bodies with arbitrarily oriented spins, through $\mathcal{O}\left(S_{1} S_{2}\right)$.

\section{EFFECTIVE FIELD THEORY AND DERIVED HAMILTONIAN}

Having found scattering amplitudes of general relativity coupled with higher-spin fields of the type described in Sec. II, we will now describe their translation to a two-body spin-dependent conservative Hamiltonian with complete velocity dependence. We will extract it from the two-to-two interaction of an effective field theory of the positiveenergy modes of higher-spin fields, thus generalizing the construction of Ref. [12] to include spin degrees of freedom. The matching procedure with spins was discussed at $\mathcal{O}(G)$ [23,63], and also at $\mathcal{O}\left(G^{2}\right)$ for spin-orbit potential expanded in velocity [23]. Here we will establish a general spinning formalism for higher orders in $G$ and all order in velocity; it has the distinct advantage of being relatively straightforward, while simultaneously producing results that allow physical observables to be obtained through standard Hamiltonian mechanics methods.

\section{A. Spin formalism}

Unlike the Lorentz-invariant setup of earlier sections, we will parametrize the spin degrees of freedom in terms of the rest-frame spin of the two fields, $\xi_{1}$ and $\xi_{2}$. Since the rest frames of the two particles are not necessarily identical (i.e., the two particles need not be simultaneously at rest), there are two copies of the little-group generators, each acting on only one of the two fields; thus, the two fields $\xi_{1}$ and $\xi_{2}$ carry little-group indices, ${ }^{11}$ which we suppress throughout. We will denote the two copies of the littlegroup generators by $\hat{\boldsymbol{S}}_{1}$ and $\hat{\boldsymbol{S}}_{2}$, respectively, and their components by $\hat{\mathrm{S}}_{a}^{i}$ with $a=1,2$. Apart from generating the $S O(3)$,

$$
\left[\hat{\mathrm{S}}_{a}^{i}, \hat{\mathrm{S}}_{b}^{j}\right]=i \delta_{a b} \epsilon^{i j k} \hat{\mathrm{S}}_{a}^{k}
$$

they also are, as in nonrelativistic quantum mechanics, the spin operators of the two particles.

We take the action of the effective field theory for the fields $\xi_{1}$ and $\xi_{2}$ to be

$$
\begin{aligned}
S= & \int_{\boldsymbol{k}} \sum_{a=1,2} \xi_{a}^{\dagger}(-\boldsymbol{k})\left(i \partial_{t}-\sqrt{\boldsymbol{k}^{2}+m_{i}^{2}}\right) \xi_{a}(\boldsymbol{k}) \\
& -\int_{\boldsymbol{k}, \boldsymbol{k}^{\prime}} \xi_{1}^{\dagger}\left(\boldsymbol{k}^{\prime}\right) \xi_{2}^{\dagger}\left(-\boldsymbol{k}^{\prime}\right) \hat{V}\left(\boldsymbol{k}^{\prime}, \boldsymbol{k}, \hat{\boldsymbol{S}}_{a}\right) \xi_{1}(\boldsymbol{k}) \xi_{2}(-\boldsymbol{k}),
\end{aligned}
$$

where we wrote the interaction term in the center-of-mass frame and $\int_{k}=\int \frac{d^{D-1} k}{(2 \pi)^{D-1}}$. As mentioned, all little-group (spin) indices are suppressed. While the field $\xi(\boldsymbol{k})$ describes a particle with momentum $\boldsymbol{k}$, its spin is always defined with respect to its own rest frame, such that the algebra in Eq. (6.1) is satisfied. This is analogous to the treatment of spinning particles in quantum mechanics, where commutation relations of spin operators are the same whether the particle is at rest or not [90]. The hat on the potential $\hat{V}\left(\boldsymbol{k}^{\prime}, \boldsymbol{k}, \hat{\boldsymbol{S}}_{a}\right)$ indicates that it is a momentum space quantity; it is a function of the incoming momentum $k$, momentum

\footnotetext{
${ }^{11}$ This is to be contrasted with the full theory, where the higherspin fields carry $S U(2)_{L} \times S U(2)_{R}$ spinor indices; cf. e.g., Eqs. (2.12) and (2.13).
} 
transfer $\boldsymbol{q}=\boldsymbol{k}-\boldsymbol{k}^{\prime}$, as well as spin operators $\hat{\boldsymbol{S}}_{1}$ and $\hat{\boldsymbol{S}}_{2}$ of the two particles.

To connect these operators with the classical rest-frame spin vectors, which is necessary in the matching of the EFT amplitudes with those of the complete theory, we take the asymptotic states of $\xi_{1}$ and $\xi_{2}$ to be spin coherent states in Eq. (2.15). Similarly, the classical two-body Hamiltonian is given by the expectation value on classical on-shell states, which satisfy $\boldsymbol{p}^{2}=(\boldsymbol{p}-\boldsymbol{q})^{2}$,

$$
\begin{aligned}
H(\boldsymbol{q}, \boldsymbol{p})= & \sqrt{\boldsymbol{p}^{2}+m_{1}^{2}}+\sqrt{\boldsymbol{p}^{2}+m_{2}^{2}} \\
& +\left\langle\boldsymbol{n}_{1} \boldsymbol{n}_{2}\left|\hat{V}\left(\boldsymbol{p}-\boldsymbol{q}, \boldsymbol{p}, \hat{\boldsymbol{S}}_{a}\right)\right| \boldsymbol{n}_{1} \boldsymbol{n}_{2}\right\rangle,
\end{aligned}
$$

where we only keep the classical part based on the counting in Eqs. (2.4) and (2.5). The spin-independent part, obtained by formally setting $\hat{\boldsymbol{S}}_{a}=0$, clearly reproduces the twobody spinless Hamiltonian. Here the momentum transfer $\boldsymbol{q}$ is the conjugate of the separation $\boldsymbol{r}$ between the two particles. Therefore the position-space classical potential follows from taking the Fourier transform with respect to $\boldsymbol{q}$,

$$
V\left(\boldsymbol{r}, \boldsymbol{p}, \boldsymbol{S}_{a}\right)=\int_{\boldsymbol{q}} e^{-i \boldsymbol{q} \cdot \boldsymbol{r}}\left\langle\boldsymbol{n}_{1} \boldsymbol{n}_{2}\left|\hat{V}\left(\boldsymbol{p}-\boldsymbol{q}, \boldsymbol{p}, \hat{\boldsymbol{S}}_{a}\right)\right| \boldsymbol{n}_{1} \boldsymbol{n}_{2}\right\rangle ;
$$

the expectation value effectively replaces the symmetric product of spin operators $\hat{\boldsymbol{S}}_{a}$ with their classical expectation values $\boldsymbol{S}_{a}$ via Eq. (2.15).

The ansatz for the interaction $\hat{V}\left(\boldsymbol{k}^{\prime}, \boldsymbol{k}, \hat{\boldsymbol{S}}_{a}\right)$, which is subsequently fixed by matching the EFT amplitudes with those of the full theory in the classical limit, is constructed such that, on the one hand, it contains only long-range interactions between the fields $\xi_{1}$ and $\xi_{2}$ and, on the other, it requires that none of the terms vanishes in the classical limit. As discussed in Refs. [12,15], this fixes the dependence on the momentum transfer at $\mathcal{O}\left(G^{n}\right)$ to be $|\boldsymbol{q}|^{n-3}$ while the dependence on the incoming momentum is arbitrary. The classical scaling, described in Eqs. (2.4) and (2.5), is the main tool for including the spin dependence. It implies that, at each $\mathcal{O}\left(G^{n}|\boldsymbol{q}|^{n-3}\right)$, we may include any number of spin operators as long as each of them is accompanied by one factor of the momentum transfer, i.e.,

$$
\begin{aligned}
\hat{V}\left(\boldsymbol{k}^{\prime}, \boldsymbol{k}, \hat{\boldsymbol{S}}_{a}\right) \supset & d^{j_{1} \cdots j_{n_{1}+n_{2}}, i_{1}, \ldots, i_{n_{1}+n_{2}}}(\boldsymbol{p}) q^{j_{1}} \cdots q^{j_{n_{1}+n_{2}}} \\
& \times \hat{\mathrm{S}}_{1}^{i_{1}} \cdots \hat{\mathrm{S}}_{1}^{i_{n_{1}}} \hat{\boldsymbol{S}}_{2}^{i_{n_{1}+1}} \cdots \hat{\mathrm{S}}_{2}^{i_{n_{1}+n_{2}}} \frac{G^{n}}{|\boldsymbol{q}|^{3-n}} .
\end{aligned}
$$

The coefficients $d^{j_{1} \cdots j_{n_{1}+n_{2}}, i_{1}, \ldots, i_{n_{1}+n_{2}}}(\boldsymbol{p})$ can be further expanded in independent tensor structures, which are constrained both by the desired/expected symmetries (such as parity) and by the classical limit. The former implies that in a parity-invariant theory an even $n_{1}+n_{2}$ requires a parity-invariant coefficient and an odd $n_{1}+n_{2}$ requires a parity-odd coefficient (i.e., one containing a Levi-Civita tensor).

The ansatz for the coefficients $d^{j_{1} \cdots j_{n_{1}+n_{2}}, i_{1}, \ldots, i_{n_{1}+n_{2}}}(\boldsymbol{p})$ is also constrained by the classical limit and the desired longrange nature of interactions. Momentum conservation implies that $\boldsymbol{p} \cdot \boldsymbol{q}=\boldsymbol{q}^{2} / 2$. Therefore, if such a contraction occurs, the corresponding term becomes subleading in the classical limit and therefore needs not be included. Similarly, if $d^{j_{1} \cdots j_{n_{1}+n_{2}}, i_{1}, \ldots, i_{n_{1}+n_{2}}}(\boldsymbol{p})$ contains a term that leads to a contraction of two of the momentum transfer factors, then that term needs not be included at $\mathcal{O}(G)$ (because it is a contact interaction) but must be included at all higher orders. Each independent tensor structure has a scalar coefficient, which depends only on the square of the center-of-mass momentum; as in [12,15], we take it to be $\boldsymbol{p}^{2}=\left(\boldsymbol{k}^{2}+\boldsymbol{k}^{2}\right) / 2$.

It is not difficult to see that at $\mathcal{O}(G)$ and in a parityinvariant theory, the only types of operators with an even and an odd number of spin operators of each particle are $\left(\boldsymbol{q} \cdot \hat{\boldsymbol{S}}_{i}\right)^{a}$ and $\boldsymbol{L}_{q} \cdot \hat{\boldsymbol{S}}_{i}\left(\boldsymbol{q} \cdot \hat{\boldsymbol{S}}_{i}\right)^{a}$, where $a$ is an even integer and

$$
\boldsymbol{L}_{q}=i(\boldsymbol{k} \times \boldsymbol{q})
$$

is the momentum space version of the orbital angular momentum. The complete set of operators that can appear in the interaction potential is the tensor product of the above sets of single-particle spin operators. All other combinations, which are proportional to $\boldsymbol{q}^{2}$ and therefore ignored at this order, must be included at $\mathcal{O}\left(G^{n \geq 2}\right)$; the construction of operators proceeds as described above.

Using the construction outlined above, we will next set up in detail the EFT to quadratic order in spin operators and through $\mathcal{O}\left(G^{2}\right)$ and determine the free coefficients of the interaction potential by matching its amplitudes with those of the full theory, summarized in Sec. VE.

\section{B. Potential bilinear in spin}

Following the framework described above, we now build the most general classical potential up to quadratic order in spins. The classical counting in Eq. (2.5) implies that, in momentum space, the possible building blocks are

linear in spin: $\boldsymbol{L}_{q} \cdot \hat{\boldsymbol{S}}_{i}$,

quadratic in spin: $\boldsymbol{q} \cdot \hat{\boldsymbol{S}}_{i} \boldsymbol{q} \cdot \hat{\boldsymbol{S}}_{j}, \quad \boldsymbol{q}^{2} \hat{\boldsymbol{S}}_{i} \cdot \hat{\boldsymbol{S}}_{j}, \quad \boldsymbol{q}^{2} \boldsymbol{k} \cdot \hat{\boldsymbol{S}}_{i} \boldsymbol{k} \cdot \hat{\boldsymbol{S}}_{j}$, $\boldsymbol{q} \cdot \boldsymbol{k} \boldsymbol{k} \cdot \hat{\boldsymbol{S}}_{i} \boldsymbol{q} \cdot \hat{\boldsymbol{S}}_{j}, \quad \boldsymbol{q} \cdot \boldsymbol{k} \boldsymbol{q} \cdot \hat{\boldsymbol{S}}_{i} \boldsymbol{k} \cdot \hat{\boldsymbol{S}}_{j}$,

where the subscripts $i, j=1,2$ are the particle labels, the prefactors are chosen such that each operator is $\mathcal{O}(1)$ under the classical counting, and $\boldsymbol{L}_{q}$, defined in (6.6), is the momentum-space version of the orbital angular momentum. Parity requires that an operator with an odd number of spins must contain a factor of $\boldsymbol{L}_{q}$. Note that the vectors $\boldsymbol{q}, \boldsymbol{k}$, 
and $\boldsymbol{L}_{q}$ span a complete basis in three dimensions. The operator $\boldsymbol{L}_{q} \cdot \hat{\boldsymbol{S}}_{i} \boldsymbol{L}_{q} \cdot \hat{\boldsymbol{S}}_{j}$ can be written in terms of the above building blocks.

If the momenta of the two particles, $\boldsymbol{k}$ and $\boldsymbol{k}^{\prime}$, are on shell, then $\boldsymbol{k} \cdot \boldsymbol{q} \rightarrow \boldsymbol{q}^{2} / 2$; consequently, the operators $\boldsymbol{q} \cdot \boldsymbol{k} \boldsymbol{k} \cdot \boldsymbol{S}_{i} \boldsymbol{q} \cdot \hat{\boldsymbol{S}}_{j}$ and $\boldsymbol{q} \cdot \boldsymbol{k} \boldsymbol{q} \cdot \hat{\boldsymbol{S}}_{i} \boldsymbol{k} \cdot \hat{\boldsymbol{S}}_{j}$ are subleading in the classical limit. This observation is similar to the removal of the products $\boldsymbol{k} \cdot \boldsymbol{q}$ in favor of $\boldsymbol{q}^{2}$ in the case of spinless particles [12]. We can see that it is even more advantageous in the presence of spin operators, because even the number of independent interactions is reduced. This choice is analogous to gauge choices in more standard derivations of twobody Hamiltonians from general relativity; as we will see shortly, it corresponds to the so-called isotropic gauge.

Thus, a minimal basis of spin-dependent interactions in the on-shell scheme, up to quadratic order in spin and linear in the spin of each particle, consists of the six operators:

$$
\begin{aligned}
\hat{\mathbb{O}}^{(0)} & =\mathbb{I}, \quad \hat{\mathbb{O}}^{(1,1)}=\boldsymbol{L}_{q} \cdot \hat{\boldsymbol{S}}_{1}, \quad \hat{\mathbb{O}}^{(1,2)}=\boldsymbol{L}_{q} \cdot \hat{\boldsymbol{S}}_{2}, \\
\hat{\mathbb{O}}^{(2,1)} & =\boldsymbol{q} \cdot \hat{\boldsymbol{S}}_{1} \boldsymbol{q} \cdot \hat{\boldsymbol{S}}_{2}, \quad \hat{\mathbb{O}}^{(2,2)}=\boldsymbol{q}^{2} \hat{\boldsymbol{S}}_{1} \cdot \hat{\boldsymbol{S}}_{2}, \\
\hat{\mathbb{O}}^{(2,3)} & =\boldsymbol{q}^{2} \boldsymbol{k} \cdot \hat{\boldsymbol{S}}_{1} \boldsymbol{k} \cdot \hat{\boldsymbol{S}}_{2} .
\end{aligned}
$$

Their expectation values in the spin coherent states, as in Eq. (6.3), are in one-to-one correspondence with the various spin-dependent monomials in the tree-level and one-loop amplitude in the full theory to this order in spin (see Sec. V E). We labeled them following the same scheme as there. The ansatz for the EFT potential operator $\hat{V}\left(\boldsymbol{k}^{\prime}, \boldsymbol{k}, \hat{\boldsymbol{S}}_{i}\right)$ to quadratic order in spin operators is

$$
\hat{V}\left(\boldsymbol{k}^{\prime}, \boldsymbol{k}, \hat{\boldsymbol{S}}_{i}\right)=\sum_{A} \hat{V}^{A}\left(\boldsymbol{k}^{\prime}, \boldsymbol{k}\right) \hat{\mathbb{O}}^{A},
$$

where $A$ runs over the superscripts of the operators in Eq. (6.8) and $\hat{V}^{A}\left(\boldsymbol{k}^{\prime}, \boldsymbol{k}\right)$ are free momentum-dependent coefficients that can be expanded as

$$
\hat{V}^{A}\left(\boldsymbol{k}^{\prime}, \boldsymbol{k}\right)=\frac{4 \pi G}{\boldsymbol{q}^{2}} d_{1}^{A}\left(\boldsymbol{p}^{2}\right)+\frac{2 \pi^{2} G^{2}}{|\boldsymbol{q}|} d_{2}^{A}\left(\boldsymbol{p}^{2}\right)+\mathcal{O}\left(G^{3}\right) .
$$

The coefficients $d_{i}^{A}$ are closely related to the $d$ coefficients in Eq. (6.5) and may be interpreted as the scalars multiplying the independent tensor structures in the latter. As mentioned in Sec. VI A and following Ref. [12], we choose the off-shell continuation $\boldsymbol{p}^{2}=\left(\boldsymbol{k}^{2}+\boldsymbol{k}^{\prime 2}\right) / 2$. While this is not important at tree level, it becomes essential for higherorder amplitudes.

It is not difficult to see that, as discussed in Sec. VI A, in the $\mathcal{O}(G)$ potential any operator $\hat{\mathbb{O}}^{A}$ which contains a factor of $\boldsymbol{q}^{2}$ can be ignored. Indeed, the $\boldsymbol{q}^{2}$ is such an operator it cancels the $\boldsymbol{q}^{-2}$ in the first term in (6.10) and thus leads to a contact term upon Fourier transform to position space. Such are $\hat{\mathbb{O}}^{(2,2)}$ and $\hat{\mathbb{O}}^{(2,3)}$, so we may therefore choose

$$
d_{1}^{(2,2)}=d_{1}^{(2,3)}=0 .
$$

Starting at $\mathcal{O}\left(G^{2}\right)$, however, the $\boldsymbol{q}^{2}$ in these operators does not cancel the nonanalytic $\boldsymbol{q}$ dependence, and therefore yields relevant long-distance effects and, in general, should not be ignored.

The position-space classical potential follows straightforwardly from the Fourier transform of $\boldsymbol{q}$ as in Eq. (6.4). (Here we strip off the coherent states.) To carry out the Fourier transform it is necessary to identify the complete dependence on $\boldsymbol{q}$. This amounts to expressing all $\boldsymbol{k}$ and $\boldsymbol{k}^{\prime}$ in terms $\boldsymbol{p}$ and $\boldsymbol{q}$; the latter may be ignored in the classical limit. This gives rise to the position-space potential

$$
V\left(\boldsymbol{r}, \boldsymbol{p}, \hat{\boldsymbol{S}}_{i}\right)=\sum_{A} V^{A}(\boldsymbol{r}, \boldsymbol{p}) \mathbb{O}^{A} .
$$

The six independent position-space operators are

$$
\begin{aligned}
\mathbb{O}^{(0)} & =\mathbb{I}, \quad \mathbb{O}^{(1,1)}=\frac{1}{\boldsymbol{r}^{2}} \boldsymbol{L} \cdot \hat{\boldsymbol{S}}_{1}, \quad \mathbb{O}^{(1,2)}=\frac{1}{\boldsymbol{r}^{2}} \boldsymbol{L} \cdot \hat{\boldsymbol{S}}_{2}, \\
\mathbb{O}^{(2,1)} & =\frac{1}{\boldsymbol{r}^{4}} \boldsymbol{r} \cdot \hat{\boldsymbol{S}}_{1} \boldsymbol{r} \cdot \hat{\boldsymbol{S}}_{2}, \quad \mathbb{O}^{(2,2)}=\frac{1}{\boldsymbol{r}^{2}} \hat{\boldsymbol{S}}_{1} \cdot \hat{\boldsymbol{S}}_{2}, \\
\mathbb{O}^{(2,3)} & =\frac{1}{\boldsymbol{r}^{2}} \boldsymbol{p} \cdot \hat{\boldsymbol{S}}_{1} \boldsymbol{p} \cdot \hat{\boldsymbol{S}}_{2},
\end{aligned}
$$

where $\boldsymbol{L}=\boldsymbol{r} \times \boldsymbol{p}$ is the orbital angular momentum and the prefactors are expanded in $G$,

$V^{A}(\boldsymbol{r}, \boldsymbol{p})=\frac{G}{|\boldsymbol{r}|} c_{1}^{A}\left(\boldsymbol{p}^{2}\right)+\left(\frac{G}{|\boldsymbol{r}|}\right)^{2} c_{2}^{A}\left(\boldsymbol{p}^{2}\right)+\mathcal{O}\left(G^{3}\right)$.

The earlier choice to trade the products $\boldsymbol{k} \cdot \boldsymbol{q}$ for $\boldsymbol{q}^{2}$ using momentum conservation translates in position space to the absence of the product $\boldsymbol{p} \cdot \boldsymbol{r}$ from the expression of the Hamiltonian. Thus, it corresponds to the so-called isotropic gauge.

The relation between the coefficients of the momentum space and position space identity operator is trivial, $c_{i}^{(0)}=d_{i}^{(0)}$. However, some of the spin operators $\hat{\mathbb{O}}^{A}$ include nontrivial (tensorlike) $\boldsymbol{q}$ dependence. Thus, the Fourier transform of $\hat{V}\left(\boldsymbol{k}^{\prime}, \boldsymbol{k}, \hat{\boldsymbol{S}}_{i}\right)$ in Eqs. (6.9) and (6.10) leads, in general, to nontrivial linear relations between the $d^{A}$ in Eq. (6.10) and the $c^{A}$ coefficients in Eq. (6.14). They are summarized in Table I. We note that, while at $\mathcal{O}(G)$ the momentum space potential depends only on $\hat{\mathbb{O}}^{(2,1)}$, its Fourier transform depends on both $\mathbb{O}^{(2,1)}$ and $\mathbb{O}^{(2,2)}$; they enter only in the combination $3 \mathbb{O}^{(2,1)}-\mathbb{O}^{(2,2)}$, which may be identified as (proportional to) the quadrupole of the system of two particles which is not inherited from the quadrupole of either one. 
TABLE I. Summary of momentum- and real-space operators for spin interactions and relations between their coefficients, through bilinear order in spins. The first column lists operator labels, $A$. The operators in momentum space and in position space, $\hat{\mathbb{O}}^{A}$ and $\mathbb{O}^{A}$, are given in columns two and three, respectively. In the last column we give the relations between the coefficients of momentum-space operators $d_{i}^{A}$ and position-space counterpart $c_{i}^{A}$ at $\mathcal{O}(G)$ and $\mathcal{O}\left(G^{2}\right)$. The momentum-space and position-space potentials are defined in Eqs. (6.10) and (6.14).

\begin{tabular}{lcccc}
\hline \hline Label $A$ & Mom. space operator & Real space operator & $\mathcal{O}(G)$ & $\mathcal{O}\left(G^{2}\right)$ \\
\hline$(0)$ & $\mathbb{I}$ & $\mathbb{I}$ & $c_{1}^{(0)}=d_{1}^{(0)}$ & $c_{2}^{(0)}=d_{2}^{(0)}$ \\
$(1,1)$ & $\boldsymbol{L}_{q} \cdot \hat{\boldsymbol{S}}_{1}$ & $\frac{1}{r^{2}} \boldsymbol{L} \cdot \hat{\boldsymbol{S}}_{1}$ & $c_{1}^{(1,1)}=-d_{1}^{(1,1)}$ & $c_{2}^{(1,1)}=-2 d_{2}^{(1,1)}$ \\
$(1,2)$ & $\boldsymbol{L}_{q} \cdot \hat{\boldsymbol{S}}_{2}$ & $\frac{1}{r^{2}} \boldsymbol{L} \cdot \hat{\boldsymbol{S}}_{2}$ & $c_{1}^{(1,2)}=-d_{1}^{(1,2)}$ & $c_{2}^{(1,2)}=-2 d_{2}^{(1,2)}$ \\
$(2,1)$ & $\boldsymbol{q} \cdot \hat{\boldsymbol{S}}_{1} \boldsymbol{q} \cdot \hat{\boldsymbol{S}}_{2}$ & $\frac{1}{\boldsymbol{r}^{4}} \boldsymbol{r} \cdot \hat{\boldsymbol{S}}_{1} \boldsymbol{r} \cdot \hat{\boldsymbol{S}}_{2}$ & $c_{1}^{(2,1)}=-3 d_{1}^{(2,1)}$ & $c_{2}^{(2,1)}=-8 d_{2}^{(2,1)}$ \\
$(2,2)$ & $\boldsymbol{q}^{2} \hat{\boldsymbol{S}}_{1} \cdot \hat{\boldsymbol{S}}_{2}$ & $\frac{1}{\boldsymbol{r}^{2}} \hat{\boldsymbol{S}}_{1} \cdot \hat{\boldsymbol{S}}_{2}$ & $c_{1}^{(2,2)}=d_{1}^{(2,1)}$ & $c_{2}^{(2,2)}=2 d_{2}^{(2,1)}-2 d_{2}^{(2,2)}$ \\
$(2,3)$ & $\boldsymbol{q}^{2} \boldsymbol{k} \cdot \hat{\boldsymbol{S}}_{1} \boldsymbol{k} \cdot \hat{\boldsymbol{S}}_{2}$ & $\frac{1}{r^{2}} \boldsymbol{k} \cdot \hat{\boldsymbol{S}}_{1} \boldsymbol{k} \cdot \hat{\boldsymbol{S}}_{2}$ & $c_{1}^{(2,3)}=0$ & $c_{2}^{(2,3)}=-2 d_{2}^{(2,3)}$ \\
\hline \hline
\end{tabular}

\section{EFT four-point scattering amplitude}

To guarantee that the EFT described above corresponds to the full theory setup and used in earlier sections and free coefficients of the EFT Lagrangian, we compare its treelevel and one-loop four-point scattering amplitude with the tree-level and one-loop amplitudes that are summarized in Sec. V E. To this end, in this section we evaluate the EFT two-to-two scattering amplitude. Before proceeding in the next section to determine its free coefficients, we use the state-of-the-art $\operatorname{spin}_{1}$-spin 2 Hamiltonians [6,54] to verify that the EFT amplitude reproduces the suitable expansion of the full theory amplitudes.

Given the simple structure of the EFT Lagrangian, it is straightforward to derive the Feynman rules. The propagator and vertices are

$$
\stackrel{(E, \boldsymbol{k})}{\longrightarrow}=\frac{i \mathbb{I}}{E-\sqrt{\boldsymbol{k}^{2}+m^{2}}+i \epsilon}
$$

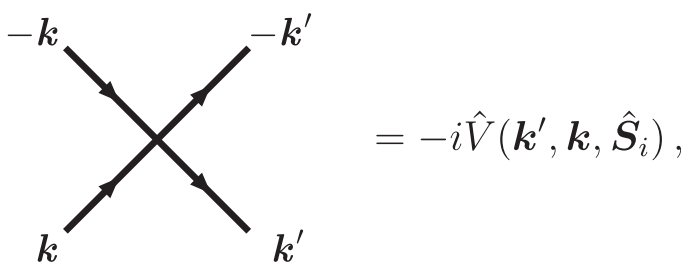

where $\mathbb{I}$ in the numerator of the propagator is an identity operator. As emphasized above, the vertices should be viewed as operators whose ordering is important. As in the spinless case, off-shell continuation of the potential needs to be defined in order to have consistent amplitude. We use $\boldsymbol{p}^{2}=\left(\boldsymbol{k}^{2}+\boldsymbol{k}^{\prime 2}\right) / 2$ in the coefficients $d_{i}^{(A)}\left(\boldsymbol{p}^{2}\right)$, and also choose Eq. (6.8) as the off-shell definitions for the operators.

To illustrate the calculation, consider the amplitude up to $\mathcal{O}\left(G^{2}\right)$. The two relevant Feynman diagrams are given in Fig. 10. It is not difficult to see that the Feynman rules give the following expression for the two-to-two scattering amplitude stripped of external-state spinors:

$$
\begin{aligned}
\widehat{\mathcal{M}}^{\mathrm{EFT}} & =-\hat{V}\left(\boldsymbol{p}^{\prime}, \boldsymbol{p}\right)-\int_{\boldsymbol{k}} \int \frac{d \omega}{2 \pi} \frac{\hat{V}\left(\boldsymbol{p}^{\prime}, \boldsymbol{k}\right) \hat{V}(\boldsymbol{k}, \boldsymbol{p})}{\left(E_{1}+\omega-\sqrt{\boldsymbol{k}^{2}+m_{1}^{2}}\right)\left(E_{2}-\omega-\sqrt{\boldsymbol{k}^{2}+m_{2}^{2}}\right)} \\
& =-\hat{V}\left(\boldsymbol{p}^{\prime}, \boldsymbol{p}\right)-\int_{\boldsymbol{k}} \frac{\hat{V}\left(\boldsymbol{p}^{\prime}, \boldsymbol{k}\right) \hat{V}(\boldsymbol{k}, \boldsymbol{p})}{E_{1}+E_{2}-\sqrt{\boldsymbol{k}^{2}+m_{1}^{2}}-\sqrt{\boldsymbol{k}^{2}+m_{2}^{2}}} .
\end{aligned}
$$

The second line is obtained by carrying out the $\omega$ integral, using the standard $i \epsilon$ prescription.

Unlike the case of spinless particles, the potential $V$ entering each vertex is an operator, and therefore the order of $\hat{V}\left(\boldsymbol{p}^{\prime}, \boldsymbol{k}\right)$ and $\hat{V}(\boldsymbol{k}, \boldsymbol{p})$ in the numerator is essential. As typically done in quantum mechanics and quantum field theory, they are ordered from left to right, beginning with the vertex adjacent to the final state, followed by the one 


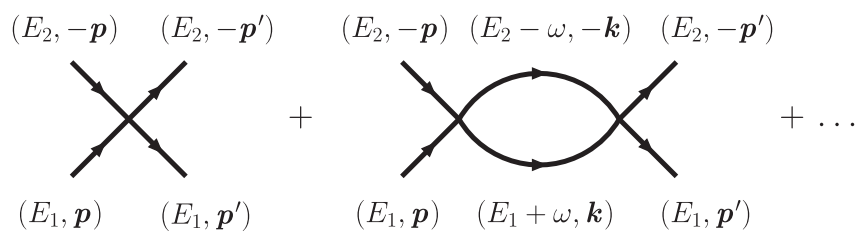

FIG. 10. The EFT scattering amplitude is given by the sum of bubble diagrams. We use the center-of-mass frame for external kinematics. The loop momentum $\boldsymbol{k}=\boldsymbol{p}+\boldsymbol{l}$ where $\boldsymbol{l}$ is the momentum transfer flowing downward in the leftmost vertex of the second diagram.

adjacent to the initial state. In terms of the one-loop Feynman graph in Fig. 10, vertices are read against the arrows denoting the momentum (and charge) flow. Each vertex brings spin operators of the same particle. In close similarity with the full theory, to consistently take the classical limit it is necessary to decompose such products in irreducible representations of the rotation group ${ }^{12}$; this is done by repeated use of the $S O(3)$ algebra in Eq. (6.1). For example, a product of two spin operators is organized as

$\hat{\mathbf{S}}_{a}^{i} \hat{\mathbf{S}}_{a}^{j}=\frac{1}{2}\left\{\hat{\mathbf{S}}_{a}^{i}, \hat{\mathbf{S}}_{a}^{j}\right\}+\frac{1}{2}\left[\hat{\mathbf{S}}_{a}^{i}, \hat{\mathbf{S}}_{a}^{j}\right]=\frac{1}{2}\left\{\hat{\mathbf{S}}_{a}^{i}, \hat{\mathrm{S}}_{a}^{j}\right\}+\frac{i}{2} \epsilon^{i j k} \hat{\mathbf{S}}_{a}^{k} ;$

this is similar with Eq. (3.13) in the full theory, written there for the generators of the Lorentz group. Although the commutator may appear to be subleading in classical counting, it can still yield relevant contributions when it appears in a loop diagram.

The propagator in Eq. (6.16) simplifies when expanded around the classical limit [12],

$$
\begin{gathered}
\frac{1}{E_{1}+E_{2}-\sqrt{\boldsymbol{k}^{2}+m_{1}^{2}}-\sqrt{\boldsymbol{k}^{2}+m_{2}^{2}}} \\
=-\frac{2 \xi E}{\boldsymbol{k}^{2}-\boldsymbol{p}^{2}}-\frac{1-3 \xi}{2 \xi E}+\cdots,
\end{gathered}
$$

where $E=E_{1}+E_{2}, \xi=E_{1} E_{2} /\left(E_{1}+E_{2}\right)^{2}$, and the ellipsis stands for higher order in classical counting which are irrelevant for $\mathcal{O}\left(G^{2}\right)$. As indicated in Fig. $10, \boldsymbol{k}=\boldsymbol{p}+\boldsymbol{l}$, where $\boldsymbol{p}$ is the center-of-mass external momentum and $\boldsymbol{l}$ is the momentum transfer in the leftmost vertex of the send diagram. We can see that the first term is $\mathcal{O}\left(|\boldsymbol{l}|^{-1}\right) \sim$ $\mathcal{O}\left(|\boldsymbol{q}|^{-1}\right)$ using $\boldsymbol{k}=\boldsymbol{p}+\boldsymbol{l}$ and expanding in $\boldsymbol{l}$. Therefore, as stated above, the commutator term in Eq. (6.17) can be relevant for the classical limit of the EFT amplitude when it interferes with the propagator. We will judiciously keep such contributions when evaluating the second term in Eq. (6.16).

\footnotetext{
${ }^{12}$ In the full theory, products of Lorentz generators were decomposed in irreducible representations of the Lorentz group.
}

The final two-to-two scattering amplitude $\mathcal{M}^{\mathrm{EFT}}$ in EFT is obtained by contracting $\widehat{\mathcal{M}}^{\mathrm{EFT}}$ with suitable externalstate states. As discussed in Secs. VI A and II, the relevant ones for the classical limit are the spin coherent states defined in Eq. (2.15). Thus,

$$
\mathcal{M}^{\mathrm{EFT}} \equiv\left\langle\boldsymbol{n}_{1}, \boldsymbol{n}_{2}\left|\widehat{\mathcal{M}}^{\mathrm{EFT}}\right| \boldsymbol{n}_{1}, \boldsymbol{n}_{2}\right\rangle
$$

Since these states are momentum independent, the net effect of the expectation value is to simply turn the spin operator $\hat{\boldsymbol{S}}_{i}$ into classical expectation values $\boldsymbol{S}_{i}$; moreover, this expectation value does not lead to any terms that are subleading in the classical limit.

At $\mathcal{O}(G)$, the EFT amplitude follows simply from evaluating the tree-level diagram in Fig. 10, which corresponds to the first term in Eq. (6.16); it is directly given by the $\mathcal{O}(G)$ potential. Keeping only the terms up to bilinear order in spin that contribute to long-range interactions, the amplitude is

$$
\begin{aligned}
\mathcal{M}_{1 \mathrm{PM}}^{\mathrm{EFT}}= & \frac{4 \pi G}{\boldsymbol{q}^{2}}\left[a_{1}^{(0)}+a_{1}^{(1,1)} \boldsymbol{L}_{q} \cdot \boldsymbol{S}_{1}+a_{1}^{(1,2)} \boldsymbol{L}_{q} \cdot \boldsymbol{S}_{2}\right. \\
& \left.+a_{1}^{(2,1)} \boldsymbol{q} \cdot \boldsymbol{S}_{1} \boldsymbol{q} \cdot \boldsymbol{S}_{2}\right] .
\end{aligned}
$$

The $a_{1}^{A}$ coefficients ${ }^{13}$ are directly given by the coefficients in the momentum-space potential (6.10),

$$
a_{1}^{A}=-d_{1}^{A}
$$

As discussed in Sec. II, a simple rule for tracking relevance in the classical limit, is that each power of spin comes with a single power of $\boldsymbol{q}$ relative to the spinless case; terms with higher powers are irrelevant. If the required powers of $\boldsymbol{q}$ appear as $\boldsymbol{q}^{2}$ at this order, we can drop the contributions because they cancel the $1 / \boldsymbol{q}^{2}$ pole and will not yield longdistance contributions to the potential.

The EFT amplitude at $\mathcal{O}\left(G^{2}\right)$ order receives contributions from both terms in Eq. (6.16) and can be written as

\footnotetext{
${ }^{13}$ These EFT amplitude coefficients are formally distinct from the full theory coefficients. However, for the EFT to correspond to the full theory, the scattering amplitudes of the two theories must be the same. Enforcing this condition, referred to as "EFT matching" which we will do in Sec. VID, leads to amplitudes' coefficients being equal, so we use the same notation for both of them.
} 


$$
\begin{aligned}
\mathcal{M}_{2 \mathrm{PM}}^{\mathrm{EFT}}= & \frac{2 \pi^{2} G^{2}}{|\boldsymbol{q}|}\left[a_{2}^{(0)}+a_{2}^{(1,1)} \boldsymbol{L}_{q} \cdot \boldsymbol{S}_{1}+a_{2}^{(1,2)} \boldsymbol{L}_{q} \cdot \boldsymbol{S}_{2}\right. \\
& +a_{2}^{(2,1)} \boldsymbol{q} \cdot \boldsymbol{S}_{1} \boldsymbol{q} \cdot \boldsymbol{S}_{2}+a_{2}^{(2,2)} \boldsymbol{q}^{2} \boldsymbol{S}_{1} \cdot \boldsymbol{S}_{2} \\
& \left.+a_{2}^{(2,3)} \boldsymbol{q} \boldsymbol{p} \cdot \boldsymbol{S}_{1} \boldsymbol{p} \cdot \boldsymbol{S}_{2}\right] \\
& +(4 \pi G)^{2} a_{\mathrm{iter}} \int \frac{d^{D-1} \ell}{(2 \pi)^{D-1}} \frac{2 \xi E}{\boldsymbol{\ell}^{\mathbf{2}}(\boldsymbol{\ell}+\boldsymbol{q})^{2}\left(\boldsymbol{\ell}^{2}+2 \boldsymbol{p} \cdot \boldsymbol{\ell}\right)}
\end{aligned}
$$

where we have expanded in $\boldsymbol{q}$ and kept only terms that are relevant in the classical limit. The first two lines are of order $1 /|\boldsymbol{q}|$, including the scaling of the spin vectors; this is the expected order of the classical potential at $\mathcal{O}\left(G^{2}\right)$. The integral in the last term originates from the one-loop diagram in Fig. 10; since the two vertices are identical and given by the $\mathcal{O}(G)$ potential, we refer to this term as an "iteration." It is not difficult to see that the integral is of order $1 / \boldsymbol{q}^{2}$. Thus, for the amplitude to be accurate to $\mathcal{O}(1 /|\boldsymbol{q}|)$, an extra power of $\boldsymbol{q}$ beyond the leading contribution must be kept in the coefficient $a_{\text {iter }}$. While this part of the amplitude does not contain information beyond the one in the tree-level potential, it is nevertheless very important. Similar to the spinless EFT, the iteration part of the EFT one-loop amplitude is IR divergent. Since the EFT is constructed to match the low-energy part of the desired complete theory, the IR divergences in EFT should be the same as in the full theory one-loop amplitude. ${ }^{14}$ In doing so the first subleading terms in the $a_{\text {iter }}$ coefficients are crucial and the required match can be enforced by appropriately choosing the subleading terms in the relation between the Lorentz generators and the spin tensor in the full theory. We emphasize that the only effect of such a choice is to guarantee the match of IR divergences and has no consequence on the finite part of the amplitude and on the $\mathcal{O}\left(G^{2}\right)$ potential.

To write compact expressions for the coefficients of the 2PM EFT amplitude in terms of those of the momentum space Hamiltonian it is convenient to define the functions

$$
\begin{aligned}
& A_{0}[X]=\left[(1-3 \xi)+2 \xi^{2} E^{2} \partial\right] X, \\
& A_{1}[X]=\left[(1-3 \xi)+\frac{2 \xi^{2} E^{2}}{\boldsymbol{p}^{2}}+2 \xi^{2} E^{2} \partial\right] X, \\
& A_{2}[X]=\left[\frac{1}{4}(1-3 \xi)+\frac{\xi^{2} E^{2}}{\boldsymbol{p}^{2}}+\frac{1}{2} \xi^{2} E^{2} \partial\right] X, \\
& A_{3}[X]=\left[\frac{3}{4}(1-3 \xi)+\frac{\xi^{2} E^{2}}{\boldsymbol{p}^{2}}+\frac{3}{2} \xi^{2} E^{2} \partial\right] X,
\end{aligned}
$$

where $\partial=\partial / \partial \boldsymbol{p}^{2}$. Then, the EFT amplitude coefficients in Eq. (6.22) are

$$
\begin{aligned}
a_{2}^{(0)} & =-d_{2}^{(0)}+\frac{1}{2 \xi E} A_{0}\left[\left(d_{1}^{(0)}\right)^{2}\right], \\
a_{2}^{(1, i)} & =-d_{2}^{(1, i)}+\frac{1}{2 \xi E} A_{1}\left[d_{1}^{(0)} d_{1}^{(1, i)}\right], \\
a_{2}^{(2,1)} & =-d_{2}^{(2,1)}+\frac{1}{2 \xi E} A_{3}\left[d_{1}^{(0)} d_{1}^{(2,1)}\right]+\frac{p^{2}}{2 \xi E} A_{2}\left[d_{1}^{(1,1)} d_{1}^{(1,2)}\right]+\frac{\xi E}{8}\left(d_{1}^{(1,1)}+d_{1}^{(1,2)}\right) d_{1}^{(2,1)}, \\
a_{2}^{(2,2)} & =-d_{2}^{(2,2)}-\frac{1}{2 \xi E} A_{2}\left[d_{1}^{(0)} d_{1}^{(2,1)}\right]-\frac{\boldsymbol{p}^{2}}{\xi E} A_{2}\left[d_{1}^{(1,1)} d_{1}^{(1,2)}\right]+\frac{\xi E}{8}\left(d_{1}^{(1,1)}+d_{1}^{(1,2)}\right) d_{1}^{(2,1)}, \\
a_{2}^{(2,3)} & =-d_{2}^{(2,3)}+\frac{\xi E}{\boldsymbol{p}^{4}}\left[d_{1}^{(0)} d_{1}^{(2,1)}\right]+\frac{1}{\xi E} A_{2}\left[d_{1}^{(1,1)} d_{1}^{(1,2)}\right]-\frac{\xi E}{2 \boldsymbol{p}^{2}}\left(d_{1}^{(1,1)}+d_{1}^{(1,2)}\right) d_{1}^{(2,1)} .
\end{aligned}
$$

Recalling that the first superscript of the Hamiltonian coefficients represents the number of spin operators in the corresponding operator, it is easy to infer that the combination $\left(d_{1}^{(1,1)}+d_{1}^{(1,2)}\right) d_{1}^{(2,1)}$ arises from three-spin terms in the numerator of the amplitude (6.16). Such terms nevertheless contribute to the two-spin terms in the amplitude through the commutator identity Eq. (6.17).

We note that the spin-dependent sector of the amplitude contains $1 / \boldsymbol{p}^{2}$ threshold singularities through the functions $A_{1,2,3}\left[d_{1}^{A} d_{1}^{B}\right]$. This singularity is physical because it appears

\footnotetext{
${ }^{14}$ Higher-loop iteration terms have a similar interpretation.
}

in the amplitude. These terms arise from the reduction to scalar integrals of various tensor integrals in the one-loop diagram in Fig. 10. This is intimately connected to the spin dependence and, as pointed out in Sec. V, has a counterpart in the amplitude calculation in the full theory. The residue of this singularity is completely fixed by $\mathcal{O}(G)$ terms in the Hamiltonian, so the singular terms should be the same in the EFT and in the full theory. The 2PM Hamiltonian obtained by demanding that the two amplitudes are identical turns out to be local in $\boldsymbol{p}$.

Last, the iteration coefficient $a_{\text {iter }}$ in Eq. (6.22) is fixed by the $\mathcal{O}(G)$ terms in the Hamiltonian: 


$$
\begin{aligned}
a_{\mathrm{iter}}= & \left(d_{1}^{(0)}\right)^{2}+d_{1}^{(0)} d_{1}^{(1,1)} \boldsymbol{L}_{q} \cdot \boldsymbol{S}_{1}+d_{1}^{(0)} d_{1}^{(1,2)} \boldsymbol{L}_{q} \cdot \boldsymbol{S}_{2}+d_{1}^{(0)} d_{1}^{(2,1)} \boldsymbol{q} \cdot \boldsymbol{S}_{1} \boldsymbol{q} \cdot \boldsymbol{S}_{2} \\
& +\frac{1}{2}\left(d_{1}^{(1,1)} d_{1}^{(1,2)}+\frac{d_{1}^{(0)} d_{1}^{(2,1)}}{\boldsymbol{p}^{2}}\right)\left[\boldsymbol{L}_{q} \cdot \boldsymbol{S}_{1} \boldsymbol{L}_{q} \cdot \boldsymbol{S}_{2}-\boldsymbol{p}^{2} \boldsymbol{q} \cdot \boldsymbol{S}_{1} \boldsymbol{q} \cdot \boldsymbol{S}_{2}\right]
\end{aligned}
$$

This expression is through $\mathcal{O}(|\boldsymbol{q}|)$, where we count $\boldsymbol{S}_{i} \sim 1 /|\boldsymbol{q}|$. In particular, the subleading terms appearing when mapping $\boldsymbol{L}_{q} \cdot \boldsymbol{S}_{1} \boldsymbol{L}_{q} \cdot \boldsymbol{S}_{2}$ to the basis in Eq. (6.7) are important.

The EFT formalism described in Sec. VI A and illustrated here for the spin bilinears also allows us to compute scattering amplitudes starting from canonical Hamiltonians in the general relativity literature. The gauge invariance of scattering amplitudes provides a straightforward test of the (in)equivalence of different-looking Hamiltonians that avoids the explicit construction of canonical transformations. Given some Hamiltonian constructed from the outset in terms of the classical spin, the calculation of amplitudes follows the same steps as above. We first promote the classical spins in the potential to operators $S_{a} \mapsto \hat{S}_{a}$. Since the classical Hamiltonian has, by construction, classical scaling, this operation does not introduce an ordering ambiguity; any such ordering, arising through Eq. (6.17), is subleading. The amplitudes then follow from the Feynman rules in Eq. (6.15) and the expression Eq. (6.16) for the tree-level and one-loop Feynman diagrams. As in our calculation, the ordering of the vertices is relevant once inserted in the one-loop (and higher-loop) diagrams. While our construction exploits on-shell conditions to eliminate $\boldsymbol{p} \cdot \boldsymbol{r} /|\boldsymbol{r}|$ and only keeps operators (6.13) in our position-space potential (6.12), typical GR-derived Hamiltonians depend on $\boldsymbol{p} \cdot \boldsymbol{r} /|\boldsymbol{r}|$ as well as other operators such as $\boldsymbol{p} \cdot \hat{\boldsymbol{S}}_{1} \boldsymbol{r} \cdot \hat{\boldsymbol{S}}_{2}$. It is essential that they all be kept in the off-shell vertex and that on-shell conditions are enforced only for the external states of the amplitude.

Using this approach, we can evaluate the EFT amplitude for the available post-Newtonian Hamiltonian with spinorbit and $\operatorname{spin}_{1}-\operatorname{spin}_{2}$ interactions. In the same gauge as the potential for spinless bodies in Eq. (8.41) of Ref. [6], the spin-dependent next-to-leading order Hamiltonian may be found in Eqs. (7.26)-(7.29) in Ref. [54], and the next-tonext-leading order one in Eqs. (138) and (139) of Ref. [55]. ${ }^{15}$ This counting translates to expansions up to $\mathcal{O}\left(G v^{4}\right)$ and $\mathcal{O}\left(G^{2} v^{2}\right)$ at the first and second orders in an expansion in Newton's constant, respectively. See Fig. 1 for the comparison of $\operatorname{spin}_{1}-\operatorname{spin}_{2}$ potential.

The tree-level EFT amplitude following from these Hamiltonians, through $\mathcal{O}\left(\boldsymbol{p}^{4}\right)$, has the same structure as Eq. (6.20); the coefficients are

$$
\begin{aligned}
a_{1}^{(0)} & =m_{1} m_{2}+\frac{3 m_{1}^{2}+8 m_{1} m_{2}+3 m_{2}^{2}}{2 m_{1} m_{2}} \boldsymbol{p}^{2}-\frac{5 m_{1}^{4}-18 m_{1}^{2} m_{2}^{2}+5 m_{2}^{4}}{8 m_{1}^{3} m_{2}^{3}} \boldsymbol{p}^{4}+\cdots, \\
a_{1}^{(1,1)} & =\frac{4 m_{1}+3 m_{2}}{2 m_{1}}+\frac{18 m_{1}^{2}+8 m_{1} m_{2}-5 m_{2}^{2}}{8 m_{1}^{3} m_{2}} \boldsymbol{p}^{2}-\frac{15 m_{1}^{4}+15 m_{1}^{2} m_{2}^{2}+12 m_{1} m_{2}^{3}-7 m_{2}^{4}}{16 m_{1}^{5} m_{2}^{3}} \boldsymbol{p}^{4}+\cdots, \\
a_{1}^{(2,1)} & =1+\frac{2 m_{1}^{2}+9 m_{1} m_{2}+2 m_{2}^{2}}{4 m_{1}^{2} m_{2}^{2}} \boldsymbol{p}^{2}-\frac{6 m_{1}^{4}+15 m_{1}^{3} m_{2}-4 m_{1}^{2} m_{2}^{2}+15 m_{1} m_{2}^{3}+6 m_{2}^{4}}{16 m_{1}^{4} m_{2}^{4}} \boldsymbol{p}^{4}+\cdots,
\end{aligned}
$$

where the ellipses stand for $\mathcal{O}\left(v^{n \geq 6}\right)$ and $a_{1}^{(1,2)}$ is obtained by exchanging $\left(m_{1}, \gamma_{1}\right)$ and $\left(m_{2}, \gamma_{2}\right)$ in $a_{1}^{(1,1)}$. It is not difficult to see that these expressions reproduce the coefficients of the full theory amplitude in Eqs. (5.49) and (5.58), through $\mathcal{O}\left(v^{4}\right)$.

The available Hamiltonians determine the $\mathcal{O}\left(G^{2}\right)$ amplitude only through $\mathcal{O}\left(v^{2}\right)$. The structure of the amplitude is the same as Eq. (6.22). The coefficients of the various spin-dependent monomials to the relevant order in velocity are

$$
\begin{aligned}
a_{2}^{(0)} & =3 m_{1} m_{2}\left(m_{1}+m_{2}\right)+\frac{3\left(m_{1}+m_{2}\right)\left(3 m_{1}^{2}+10 m_{1} m_{2}+3 m_{2}^{2}\right)}{4 m_{1} m_{2}} \boldsymbol{p}^{2}+\cdots, \\
a_{2}^{(1,1)} & =\frac{m_{1} m_{2}^{2}\left(4 m_{1}+3 m_{2}\right)}{2\left(m_{1}+m_{2}\right) \boldsymbol{p}^{2}}+\frac{20 m_{1}^{3}+53 m_{1}^{2} m_{2}+41 m_{1} m_{2}^{2}+9 m_{2}^{3}}{4 m_{1}\left(m_{1}+m_{2}\right)}+\frac{3\left(30 m_{1}^{4}+71 m_{1}^{3} m_{2}+43 m_{1}^{2} m_{2}^{2}-m_{1} m_{2}^{3}-4 m_{2}^{4}\right)}{16 m_{1}^{3} m_{2}\left(m_{1}+m_{2}\right)} \boldsymbol{p}^{2}+\cdots, \\
a_{2}^{(2,1)} & =\frac{m_{1}^{2} m_{2}^{2}}{2\left(m_{1}+m_{2}\right) \boldsymbol{p}^{2}}+\frac{\left(7 m_{1}^{2}+15 m_{1} m_{2}+7 m_{2}^{2}\right)}{2\left(m_{1}+m_{2}\right)}+\frac{3\left(3 m_{1}^{4}+39 m_{1}^{3} m_{2}+74 m_{1}^{2} m_{2}^{2}+39 m_{1} m_{2}^{3}+3 m_{2}^{4}\right)}{16 m_{1}^{2} m_{2}^{2}\left(m_{1}+m_{2}\right)} \boldsymbol{p}^{2}+\cdots,
\end{aligned}
$$

\footnotetext{
${ }^{15}$ See Ref. [56] for the equivalence of the spin Hamiltonian derived using NRGR.
} 


$$
\begin{aligned}
& a_{2}^{(2,2)}=-a_{2}^{(2,1)} \\
& a_{2}^{(2,3)}=\frac{m_{1}^{2} m_{2}^{2}}{\left(m_{1}+m_{2}\right) \boldsymbol{p}^{4}}+\frac{19 m_{1}^{2}+40 m_{1} m_{2}+19 m_{2}^{2}}{4\left(m_{1}+m_{2}\right) \boldsymbol{p}^{2}}+\frac{3\left(3 m_{1}^{4}+45 m_{1}^{3} m_{2}+86 m_{1}^{2} m_{2}^{2}+45 m_{1} m_{2}^{3}+3 m_{2}^{4}\right.}{16 m_{1}^{2} m_{2}^{2}\left(m_{1}+m_{2}\right)}+\cdots
\end{aligned}
$$

The ellipses stand for $\mathcal{O}\left(v^{n \geq 4}\right)$ and $a_{2}^{(1,2)}$ is obtained by interchanging $m_{1}$ and $m_{2}$ in $a_{2}^{(1,1)}$. Note that the operator associated with $a_{2}^{(2,3)}$ is $\boldsymbol{p} \cdot \hat{\boldsymbol{S}}_{1} \boldsymbol{p} \cdot \hat{\boldsymbol{S}}_{2}$, which carries $\mathcal{O}\left(\boldsymbol{p}^{2}\right)$, its coefficient is determined only though $\mathcal{O}\left(v^{0}\right)$. We also note the coefficients of the spin-dependent monomials exhibit $1 / \boldsymbol{p}^{2}$ singularities; similar to the full theory amplitude and to the calculation of the EFT amplitude from the potential (6.9), they originate from the tensor reduction of EFT oneloop integrals and their residues are controlled by $\mathcal{O}(G)$ Hamiltonian terms. The agreement outlined here serves as a highly nontrivial test of the spin EFT formalism formulated in this section and of the higher-spin field theory construction used in earlier sections.

\section{Conservative spin Hamiltonian from matching}

With the amplitudes of the EFT and of the full theory in hand, we turn to constructing the Hamiltonian. It is fixed by demanding that the two amplitudes are the same,

$$
\mathcal{M}_{1 \mathrm{PM}}^{\mathrm{EFT}}=\frac{\mathcal{M}_{4}^{\text {tree }}}{4 E_{1} E_{2}}, \quad \mathcal{M}_{2 \mathrm{PM}}^{\mathrm{EFT}}=\frac{\mathcal{M}_{4}^{1 \text { loop }}}{4 E_{1} E_{2}} .
$$

The EFT amplitude is parametrized in Eqs. (6.20) and (6.22), with coefficients given in Eqs. (6.21) and (6.24) while the full theory amplitude may be found, in the same parametrization, in Eqs. (5.49), (5.53), and (5.58). The equality of amplitudes (6.28) implies that coefficients of identical spin-dependent monomials-both denoted by $a_{i}^{A}$-are also identical. From here we extract the coefficients $d_{i}^{A}$ of the momentum-space potential.

Carrying this out at $\mathcal{O}(G)$ we find that $d_{1}^{A}$ are given by

$$
\begin{aligned}
d_{1}^{(0)}= & \frac{m^{2} \nu^{2}}{\xi \gamma^{2}}\left(1-2 \sigma^{2}\right), \\
d_{1}^{(1, i)}= & -\frac{\nu}{\xi \gamma^{2}} \frac{2 \sigma E}{m_{i}}-\frac{1}{m_{i}^{2}\left(\gamma_{i}+1\right)} d_{1}^{(0)}, \\
d_{1}^{(2,1)}= & \frac{\nu}{\xi \gamma^{2}}\left(1-2 \sigma^{2}\right)+\left(\frac{1}{m_{1}\left(\gamma_{1}+1\right)}+\frac{1}{m_{2}\left(\gamma_{2}+1\right)}\right) \frac{2 \sigma \boldsymbol{p}^{2}}{E \xi} \\
& +\frac{\boldsymbol{p}^{2}}{m_{1}^{2} m_{2}^{2}\left(\gamma_{1}+1\right)\left(\gamma_{2}+1\right)} d_{1}^{(0)}
\end{aligned}
$$

and $d_{1}^{(2,2)}=d_{1}^{(2,3)}=0$ because they do not mediate longrange interactions. The variables used here are defined in Eq. (5.50) and below Eq. (5.54). The position-space potential follows immediately via Table I:

$$
\begin{array}{rlrl}
c_{1}^{(0)} & =d_{1}^{(0)}, & c_{1}^{(1, i)}=-d_{1}^{(1, i)}, & c_{1}^{(2,1)}=-3 d_{1}^{(2,1)}, \\
c_{1}^{(2,2)}=d_{1}^{(2,1)}, & c_{1}^{(2,3)}=0 .
\end{array}
$$

At $\mathcal{O}(G)$, the amplitude and potential are directly related, so the potential retains the structure in Eq. (5.58). The relation between $c_{1}^{(2,1)}$ and $c_{1}^{(2,2)}$ implies that, at this order, the spinbilinear part of the position-space potential depends only on the two-particle quadrupole $3 \boldsymbol{r} \cdot \boldsymbol{S}_{1} \boldsymbol{r} \cdot \boldsymbol{S}_{2}-\boldsymbol{r}^{2} \boldsymbol{S}_{1} \cdot \boldsymbol{S}_{2}$.

The coefficients (6.30) determine the $\mathcal{O}(G)$ Hamiltonian to leading order in the classical limit. As discussed earlier in this section, we may consider keeping subleading terms in this Hamiltonian, as they may yield leading-order classical terms in the $\mathcal{O}\left(G^{2}\right)$ amplitude and thus modify the classical Hamiltonian at that order. Fortunately, the on-shell conditions force the correction to be either suppressed by two powers of $\boldsymbol{q}$ compared to the classical terms or proportional to the operators $\boldsymbol{q}^{2} \boldsymbol{p} \cdot \hat{\boldsymbol{S}}_{i} \boldsymbol{q} \cdot \hat{\boldsymbol{S}}_{j}$. The latter structure cancels one graviton pole, leading to a contact term which gives a vanishing contribution in the one-loop EFT amplitude. The former may yield at most contributions to the EFT amplitude that are suppressed by one power of $\boldsymbol{q}$. We conclude therefore that the coefficients (6.30) are sufficient to generate the correct EFT amplitudes through $\mathcal{O}\left(G^{2}\right)$.

As a nontrivial consistency check, which verifies that the EFT we constructed corresponds to the full theory used in earlier sections, we can compare the iteration coefficient, $a_{\text {iter }}$ in Eq. (6.25), and the classical limit of the $a_{\mathrm{B}}$ of the energy-integrated box integral. They are both determined by $\mathcal{O}(G)$ data and multiply the same IR-divergent threedimensional integral, which is $\mathcal{O}\left(|\boldsymbol{q}|^{-1}\right)$. We find

$$
\frac{d_{\mathrm{B}}}{4 E_{1} E_{2}}=(4 \pi G)^{2} 4 \xi E^{2} a_{\mathrm{iter}}+\mathcal{O}(|\boldsymbol{q}|),
$$

which is indeed required for the equality of the EFT and full theory amplitudes, Eq. (6.28), at $\mathcal{O}\left(\boldsymbol{q}^{-2}\right)$. The match of the IR divergent part can be extended to subleading order in two equivalent ways. On the one hand, we can extend the $\mathcal{O}(G)$ EFT Hamiltonian by subleading $[\mathcal{O}(|\boldsymbol{q}|)]$ terms which are adjusted for such that the equality above holds to $\mathcal{O}(|\boldsymbol{q}|)$. They are related to the fact that the relation (2.20) needed to express $d_{\mathrm{B}}$ in terms of the classical spin vector hold only to leading order in the classical limit. Conversely, we can include next-to-leading order terms in Eq. (2.20), and we verified that such terms extend the equality (6.31) to $\mathcal{O}(|\boldsymbol{q}|)$. The details of this subleading correction are not important; their only effect is to restore the equality of the 
IR-divergent part of the EFT and full-theory one-loop amplitudes without altering the classical EFT Hamiltonian through $\mathcal{O}\left(G^{2}\right)$.

The coefficients $d_{2}^{A}$ of the $\mathcal{O}\left(G^{2}\right)$ Hamiltonian are found from Eq. (6.28) with the EFT amplitude coefficients in Eq. (6.24) and full theory coefficients in Eqs. (5.53) and (5.58). The spin-independent term and spin-orbit interaction coefficients $d_{i}^{A}$ are

$$
\begin{aligned}
d_{2}^{(0)}= & \frac{\nu^{2} m^{3}}{\xi \gamma^{2}}\left[\frac{3}{4}\left(1-5 \sigma^{2}\right)-\frac{4 \nu \sigma\left(1-2 \sigma^{2}\right)}{\gamma \xi}-\frac{\nu^{2}(1-\xi)\left(1-2 \sigma^{2}\right)^{2}}{2 \gamma^{3} \xi^{2}}\right], \\
d_{2}^{(1,1)}= & \frac{\nu E}{4 \xi \gamma^{2} m_{1}}\left[-\frac{\left(5 \sigma^{2}-3\right) \sigma}{\sigma^{2}-1}\left(4 m_{1}+3 m_{2}\right)+\frac{2\left(2 \sigma^{2}-1\right)}{\sigma^{2}-1}\left(2 \sigma E\left(\gamma_{1}^{-2}+\gamma_{2}^{-2}\right)+\gamma_{1}^{-1}\left(1-2 \sigma^{2}\right) m_{2}\right)+\frac{4 \nu\left(6 \sigma^{2}-1\right) E}{\xi \gamma^{2}}\right] \\
& -\frac{1}{m_{1}^{2}\left(\gamma_{1}+1\right)} d_{2}^{(0)} .
\end{aligned}
$$

The variables used here are defined in Eq. (5.50) and below Eq. (5.54), and $d_{2}^{(1,2)}$ is obtained by interchanging $\left(m_{1}, \gamma_{1}\right)$ and $\left(m_{2}, \gamma_{2}\right)$ in $d_{2}^{(1,1)}$. The expressions for the coefficients $d_{2}^{(2,1)}, d_{2}^{(2,2)}, d_{2}^{(2,3)}$ of the spin-bilinear operators are more complicated so we provide them in the Mathematica ancillary file coefficients.m.

The coefficient functions of the position-space potential are readily obtained through the relations in Table I:

$$
c_{2}^{(0)}=d_{2}^{(0)}, \quad c_{2}^{(1, i)}=-2 d_{2}^{(1, i)}, \quad c_{2}^{(2,1)}=-8 d_{2}^{(2,1)}, \quad c_{2}^{(2,2)}=2 d_{2}^{(2,1)}-2 d_{2}^{(2,2)}, \quad c_{2}^{(2,3)}=-2 d_{2}^{(2,3)} .
$$

We can verify that the probe limit of our all-orders-in-velocity Hamiltonian reproduces the results of Ref. [75], where the Hamiltonian was constructed in this limit up to quadratic order in spins. (See also Refs. [65,91].) Taking $m_{1},|\boldsymbol{p}| \ll m_{2}$, so we have a spinning probe particle 1 in a Kerr background by particle 2 and mapping those results into isotropic gauge, ${ }^{16}$ the probe-limit real-space potential up to bilinear order in spin is

$$
\begin{aligned}
V\left(\boldsymbol{p}, \boldsymbol{r}, \boldsymbol{S}_{i}\right)= & \left(\frac{G m_{2}}{m_{1}} \frac{\left(2 \gamma_{1}+1\right)}{\gamma_{1}\left(\gamma_{1}+1\right)}-\frac{G^{2} m_{2}^{2}}{m_{1}} \frac{\left(9 \gamma_{1}^{3}+7 \gamma_{1}^{2}+2 \gamma_{1}+2\right)}{2 r \gamma_{1}^{3}\left(\gamma_{1}+1\right)}\right) \frac{\boldsymbol{L} \cdot \boldsymbol{S}_{1}}{r^{3}}+\left(2 G-\frac{6 G^{2} m_{2}}{r}\right) \frac{\boldsymbol{L} \cdot \boldsymbol{S}_{2}}{r^{3}} \\
& +G \frac{\left(2 \gamma_{1}-1\right)}{\gamma_{1}} \frac{1}{r^{5}}\left(3 \boldsymbol{r} \cdot \boldsymbol{S}_{1} \boldsymbol{r} \cdot \boldsymbol{S}_{2}-\boldsymbol{r}^{2} \boldsymbol{S}_{1} \cdot \boldsymbol{S}_{2}\right)+G^{2} m_{2} \frac{\left(-40 \gamma_{1}^{4}-28 \gamma_{1}^{3}+14 \gamma_{1}^{2}+6 \gamma_{1}+6\right)}{2 \gamma_{1}^{3}\left(\gamma_{1}+1\right)} \frac{\boldsymbol{S}_{1} \boldsymbol{r} \cdot \boldsymbol{S}_{2}}{r^{6}} \\
& +G^{2} m_{2} \frac{\left(16 \gamma_{1}^{4}+13 \gamma_{1}^{3}-5 \gamma_{1}^{2}-2 \gamma_{1}-2\right)}{2 \gamma_{1}^{3}\left(\gamma_{1}+1\right)} \frac{\boldsymbol{S}_{1} \cdot \boldsymbol{S}_{2}}{r^{4}}+G^{2} \frac{m_{2}}{m_{1}^{2}} \frac{\left(\gamma_{1}-1\right)}{2 \gamma_{1}\left(\gamma_{1}+1\right)^{2}} \frac{\boldsymbol{p} \cdot \boldsymbol{S}_{1} \boldsymbol{p} \cdot \boldsymbol{S}_{2}}{r^{4}} .
\end{aligned}
$$

Only the leading contribution in small $m_{1} / m_{2}$ is kept for each type of spin-dependent monomial. Mapping the expression above to the form in Eq. (6.12) and Eq. (6.14) yields the coefficient $c_{i}^{A}$. We can see that the $\mathcal{O}(G)$ position-space potential contains only the combination $3 \boldsymbol{r} \cdot \boldsymbol{S}_{1} \boldsymbol{r} \cdot \boldsymbol{S}_{2}-\boldsymbol{r}^{2} \boldsymbol{S}_{1} \cdot \boldsymbol{S}_{2}$, in agreement with our result [cf. the discussion below Eq. (6.30)]. Equation (6.34) is in complete agreement with the probe limit of our potential.

We note that the combination $3 \boldsymbol{r} \cdot \boldsymbol{S}_{1} \boldsymbol{r} \cdot \boldsymbol{S}_{2}-\boldsymbol{r}^{2} \boldsymbol{S}_{1} \cdot \boldsymbol{S}_{2}$, as a symmetric traceless tensor in $\boldsymbol{r}$, can be interpreted as the quadrupole of the two-particle system which is not induced by the quadrupole of the individual constituents, and thus it is entirely due to their interaction. It may therefore be natural to organize the spin dependence in terms of this operator, even at higher orders in $G$. At $\mathcal{O}\left(G^{n}\right)$ it can also be identified as the traceless-symmetric structure in the momentum space Hamiltonian, $|\boldsymbol{q}|^{n-3}\left(3 \boldsymbol{q} \cdot \boldsymbol{S}_{1} \boldsymbol{q} \cdot \boldsymbol{S}_{2}-\boldsymbol{q}^{2} \boldsymbol{S}_{1} \cdot \boldsymbol{S}_{2}\right)$; while the second term drops out of the $\mathcal{O}(G)$ Hamiltonian (because it represents a contact interaction), the Fourier transform of the remainder, including the additional $\boldsymbol{q}$-dependent factors, is the operator on the second line of Eq. (6.34).

\section{E. Summary of EFT formulas}

Here we collect the formulas that define the EFT constructed in this section and its coefficient functions determined by matching its amplitudes with those of the full theory.

\footnotetext{
${ }^{16}$ We thank Justin Vines for sharing this result.
} 
Our real-space Hamiltonian is

$H=H^{(0)}\left(r^{2}, p^{2}\right)+H^{(1, i)}\left(r^{2}, p^{2}\right) \boldsymbol{L} \cdot \boldsymbol{S}_{i}+H^{(2,1)}\left(r^{2}, p^{2}\right) \boldsymbol{r} \cdot \boldsymbol{S}_{1} \boldsymbol{r} \cdot \boldsymbol{S}_{2}+H^{(2,2)}\left(r^{2}, p^{2}\right) \boldsymbol{S}_{1} \cdot \boldsymbol{S}_{2}+H^{(2,3)}\left(r^{2}, p^{2}\right) \boldsymbol{p} \cdot \boldsymbol{S}_{1} \boldsymbol{p} \cdot \boldsymbol{S}_{2}+\cdots$,

where the ellipsis stands for terms quadratic in the spin of each particle as well as for terms of higher orders in spin. This Hamiltonian corresponds to the one in Eqs. (6.12) and (6.13). As usual, the coefficients in Eq. (6.35) can be expanded in Newton's constant $G$, as in Eq. (6.14):

$$
\begin{aligned}
H^{(0)}\left(r^{2}, p^{2}\right) & =\sqrt{\boldsymbol{p}^{2}+m_{1}^{2}}+\sqrt{\boldsymbol{p}^{2}+m_{2}^{2}}+\frac{G}{r} c_{1}^{(0)}\left(\boldsymbol{p}^{2}\right)+\left(\frac{G}{r}\right)^{2} c_{2}^{(0)}\left(\boldsymbol{p}^{2}\right)+\mathcal{O}\left(G^{3}\right), \\
H^{(1, i)}\left(r^{2}, p^{2}\right) & =\frac{1}{r^{2}}\left[\frac{G}{r} c_{1}^{(1, i)}\left(\boldsymbol{p}^{2}\right)+\left(\frac{G}{r}\right)^{2} c_{2}^{(1, i)}\left(\boldsymbol{p}^{2}\right)+\mathcal{O}\left(G^{3}\right)\right] \\
H^{(2,1)}\left(r^{2}, p^{2}\right) & =\frac{1}{r^{4}}\left[\frac{G}{r} c_{1}^{(2,1)}\left(\boldsymbol{p}^{2}\right)+\left(\frac{G}{r}\right)^{2} c_{2}^{(2,1)}\left(\boldsymbol{p}^{2}\right)+\mathcal{O}\left(G^{3}\right)\right] \\
H^{(2,2)}\left(r^{2}, p^{2}\right) & =\frac{1}{r^{2}}\left[\frac{G}{r} c_{1}^{(2,2)}\left(\boldsymbol{p}^{2}\right)+\left(\frac{G}{r}\right)^{2} c_{2}^{(2,2)}\left(\boldsymbol{p}^{2}\right)+\mathcal{O}\left(G^{3}\right)\right] \\
H^{(2,3)}\left(r^{2}, p^{2}\right) & =\frac{1}{r^{2}}\left[\frac{G}{r} c_{1}^{(2,3)}\left(\boldsymbol{p}^{2}\right)+\left(\frac{G}{r}\right)^{2} c_{2}^{(2,3)}\left(\boldsymbol{p}^{2}\right)+\mathcal{O}\left(G^{3}\right)\right]
\end{aligned}
$$

The first coefficient function, $H^{(0)}\left(r^{2}, p^{2}\right)$, is the Hamiltonian that describes the gravitational interaction of spinless particles; the remaining ones give systematically spin-dependent interactions. The coefficients $c_{i}^{A}$ are the same as in Eq. (6.14).

From Eqs. (6.29) and (6.30) we have the explicit values of the $\mathcal{O}(G)$ position-space Hamiltonian coefficients:

$$
\begin{aligned}
c_{1}^{(0)} & =\frac{m^{2} \nu^{2}}{\xi \gamma^{2}}\left(1-2 \sigma^{2}\right), \\
c_{1}^{(1, i)} & =\frac{\nu}{\xi \gamma^{2}} \frac{2 \sigma E}{m_{i}}+\frac{1}{m_{i}^{2}\left(\gamma_{i}+1\right)} c_{1}^{(0)}, \\
c_{1}^{(2,1)} & =-\frac{3 \nu}{\xi \gamma^{2}}\left(1-2 \sigma^{2}\right)-\left(\frac{3}{m_{1}\left(\gamma_{1}+1\right)}+\frac{3}{m_{2}\left(\gamma_{2}+1\right)}\right) \frac{2 \sigma \boldsymbol{p}^{2}}{E \xi}-\frac{3 \boldsymbol{p}^{2}}{m_{1}^{2} m_{2}^{2}\left(\gamma_{1}+1\right)\left(\gamma_{2}+1\right)} c_{1}^{(0)}, \\
c_{1}^{(2,2)} & =-\frac{1}{3} c_{1}^{(2,1)}, \\
c_{1}^{(2,3)} & =0 .
\end{aligned}
$$

Similarly, the $\mathcal{O}\left(G^{2}\right)$ terms are obtained from Eqs. (6.32) and (6.33). The spin-independent and spin-orbit ones are given by

$$
\begin{aligned}
c_{2}^{(0)}= & \frac{\nu^{2} m^{3}}{\xi \gamma^{2}}\left[\frac{3}{4}\left(1-5 \sigma^{2}\right)-\frac{4 \nu \sigma\left(1-2 \sigma^{2}\right)}{\gamma \xi}-\frac{\nu^{2}(1-\xi)\left(1-2 \sigma^{2}\right)^{2}}{2 \gamma^{3} \xi^{2}}\right], \\
c_{2}^{(1,1)}= & -\frac{\nu E}{2 \xi \gamma^{2} m_{1}}\left[-\frac{\left(5 \sigma^{2}-3\right) \sigma}{\sigma^{2}-1}\left(4 m_{1}+3 m_{2}\right)+\frac{2\left(2 \sigma^{2}-1\right)}{\sigma^{2}-1}\left(2 \sigma E\left(\gamma_{1}^{-2}+\gamma_{2}^{-2}\right)+\gamma_{1}^{-1}\left(1-2 \sigma^{2}\right) m_{2}\right)\right. \\
& \left.+\frac{4 \nu\left(6 \sigma^{2}-1\right) E}{\xi \gamma^{2}}\right]+\frac{2}{m_{1}^{2}\left(\gamma_{1}+1\right)} c_{2}^{(0)}, \\
c_{2}^{(1,2)}= & \left.c_{2}^{(1,1)}\right|_{m_{1} \leftrightarrow m_{2}, \gamma_{1} \leftrightarrow \gamma_{2}} .
\end{aligned}
$$

The remaining lengthier coefficients $c_{2}^{(2,1)}, c_{2}^{(2,2)}$, and $c_{2}^{(2,3)}$ are found in the Supplemental Material [92]. The variables used here are defined in Eq. (5.50) and below Eq. (5.54): 


$$
\begin{aligned}
& \gamma=\frac{E}{m}, \quad \gamma_{1}=\frac{E_{1}}{m_{1}}, \quad \gamma_{2}=\frac{E_{2}}{m_{2}}, \quad \xi=\frac{E_{1} E_{2}}{E^{2}}, \quad \sigma=\frac{p_{1} \cdot p_{2}}{m_{1} m_{2}}, \\
& E=E_{1}+E_{2}, \quad m=m_{1}+m_{2}, \quad \nu=\frac{m_{1} m_{2}}{m^{2}}
\end{aligned}
$$

where $E_{1}$ and $E_{2}$ are the energies of the two particles.

The EFT amplitude coefficients can be obtained from the real-space Hamiltonian coefficients from Table I and Eqs. (6.21) and (6.24). At $\mathcal{O}(G)$ we have

$$
\begin{gathered}
a_{1}^{(0)}=-c_{1}^{(0)}, \quad a_{1}^{(1,1)}=c_{1}^{(1,1)}, \quad a_{1}^{(1,2)}=c_{1}^{(1,2)}, \\
a_{1}^{(2,1)}=\frac{1}{3} c_{1}^{(2,1)}=-c_{1}^{(2,2)}, \quad a_{1}^{(2,2)}=0, \quad a_{1}^{(2,3)}=0,
\end{gathered}
$$

while at $\mathcal{O}\left(G^{2}\right)$ we have

$$
\begin{aligned}
a_{2}^{(0)} & =-c_{2}^{(0)}+\frac{1}{2 \xi E} A_{0}\left[\left(c_{1}^{(0)}\right)^{2}\right] \\
a_{2}^{(1, i)} & =\frac{1}{2} c_{2}^{(1, i)}-\frac{1}{2 \xi E} A_{1}\left[c_{1}^{(0)} c_{1}^{(1, i)}\right], \\
a_{2}^{(2,1)} & =\frac{1}{8} c_{2}^{(2,1)}-\frac{1}{6 \xi E} A_{3}\left[c_{1}^{(0)} c_{1}^{(2,1)}\right]+\frac{p^{2}}{2 \xi E} A_{2}\left[c_{1}^{(1,1)} c_{1}^{(1,2)}\right]+\frac{\xi E}{24}\left(c_{1}^{(1,1)}+c_{1}^{(1,2)}\right) c_{1}^{(2,1)}, \\
a_{2}^{(2,2)} & =\frac{1}{8} c_{2}^{(2,1)}+\frac{1}{2} c_{2}^{(2,2)}+\frac{1}{6 \xi E} A_{2}\left[c_{1}^{(0)} c_{1}^{(2,1)}\right]-\frac{p^{2}}{\xi E} A_{2}\left[c_{1}^{(1,1)} c_{1}^{(1,2)}\right]+\frac{\xi E}{24}\left(c_{1}^{(1,1)}+c_{1}^{(1,2)}\right) c_{1}^{(2,1)}, \\
a_{2}^{(2,3)} & =\frac{1}{2} c_{2}^{(2,3)}-\frac{\xi E}{3 \boldsymbol{p}^{4}}\left[c_{1}^{(0)} c_{1}^{(2,1)}\right]+\frac{1}{\xi E} A_{2}\left[c_{1}^{(1,1)} c_{1}^{(1,2)}\right]-\frac{\xi E}{6 \boldsymbol{p}^{2}}\left(c_{1}^{(1,1)}+c_{1}^{(1,2)}\right) c_{1}^{(2,1)},
\end{aligned}
$$

where

$$
\begin{aligned}
& A_{0}[X]=\left[(1-3 \xi)+2 \xi^{2} E^{2} \partial\right] X, \\
& A_{1}[X]=\left[(1-3 \xi)+\frac{2 \xi^{2} E^{2}}{p^{2}}+2 \xi^{2} E^{2} \partial\right] X, \\
& A_{2}[X]=\left[\frac{1}{4}(1-3 \xi)+\frac{\xi^{2} E^{2}}{p^{2}}+\frac{1}{2} \xi^{2} E^{2} \partial\right] X, \\
& A_{3}[X]=\left[\frac{3}{4}(1-3 \xi)+\frac{\xi^{2} E^{2}}{p^{2}}+\frac{3}{2} \xi^{2} E^{2} \partial\right] X .
\end{aligned}
$$

The explicit values of the amplitude coefficients $a_{i}^{A}$ summarized in Sec. VE are much simpler than the corresponding $c_{i}^{A}$ Hamiltonian coefficients. This is not surprising given that Hamiltonians are gauge dependent, while amplitudes are gauge independent. It would be interesting to see if there exists a formulation of the EFT which leads to a Hamiltonian which exhibits the simplicity of the scattering amplitude.

\section{PHYSICAL OBSERVABLES}

The two-body Hamiltonian constructed in the previous section allows us to compute observables of scattering processes and of bound motion of spinning bodies. We will focus here on scattering observables, to point out a simple connection to the eikonal phase. Unlike the spinless case where the motion occurs in a plane and therefore there is only a single scattering angle, the spinning case has several interesting observables. Since the orbital angular momentum is no longer conserved, the scattering process is threedimensional, and thus there are two deflection angles. Moreover, since the spins are not separately conserved, they also change in a scattering process. As for the spinless case, these scattering observables are useful stepping stones for the construction of effective one-body Hamiltonians $[2,93,94]$ which can be used to evaluate bound-state dynamics. In this case, the dynamics is similarly rich with three-dimensional motion and multiple angles, leading to nontrivial modulation of gravitational wave signals which may be used to determine the properties of the binary constituents.

\section{A. Classical mechanics of particles with spin}

Consider the general problem of an arbitrary Hamiltonian describing the interaction of two particles with rest-frame spin three-vectors $\boldsymbol{S}_{1}$ and $\boldsymbol{S}_{2}$ in their centerof-mass frame, $H=H\left(\boldsymbol{r}, \boldsymbol{p}, \boldsymbol{S}_{1}, \boldsymbol{S}_{2}\right)$. In our case we will truncate to a fixed number of spin vectors, namely bilinear in spin. This is consistent, since terms in the solutions to the 
equations of motion with a certain number of spin vectors do not receive contributions from terms in the Hamiltonian with a larger number of spin vectors. While, as usual, $\boldsymbol{r}$ and $\boldsymbol{p}$ are canonically conjugate to each other, the spin variables do not have a natural canonical conjugate. To derive the equations of motion we use the fact that they must generate $S O(3)$, so

$$
\left\{S_{a}^{i}, S_{b}^{j}\right\}=\delta_{a b} \epsilon^{i j k} S_{a}^{k}, \quad a, b=1,2,
$$

where $\{A, B\}$ is the Poisson bracket of $A$ and $B$. One way to understand this relation is by recalling that in the complete theory the spin vector is given by the expectation value of the Lorentz generator. Applying Ehrenfest's theorem to the Lorentz algebra leads to Eq. (7.1). A similar strategy for deriving the equations of motion for the spin variables is found in, for example, Refs. [21,93].

The equations of motion are then

$\dot{\boldsymbol{r}}=\frac{\partial H}{\partial \boldsymbol{p}}, \quad \dot{\boldsymbol{p}}=-\frac{\partial H}{\partial \boldsymbol{r}}, \quad \dot{\boldsymbol{S}}_{a}=-\boldsymbol{S}_{a} \times \frac{\partial H}{\partial \boldsymbol{S}_{a}}, \quad a=1,2$,

where in the spin equation of motion no summation is implied on the right-hand side. One can use spherical polar coordinates, but for the purpose of finding the impulse $\Delta p$ we find it convenient to use Cartesian coordinates. One may either solve the equations of motion for coordinates and momenta and spins as a function of time or one may choose the $z$ coordinate as an effective time parameter.

There are two conservation laws that aid the construction of classical solutions. These fix the energy and the total angular momentum in terms of their asymptotic values:

$$
\begin{aligned}
& E=H\left(\boldsymbol{r}, \boldsymbol{p}, \boldsymbol{S}_{1}, \boldsymbol{S}_{2}\right)=\sqrt{p_{\infty}^{2}+m_{1}^{2}}+\sqrt{p_{\infty}^{2}+m_{2}^{2}}, \\
& \boldsymbol{I}=\boldsymbol{r} \times \boldsymbol{p}+\boldsymbol{S}_{1}+\boldsymbol{S}_{2}=\boldsymbol{L}_{\mathrm{in}}+\boldsymbol{S}_{1, \text { in }}+\boldsymbol{S}_{2, \text { in }},
\end{aligned}
$$

where $\boldsymbol{p}_{\infty}=p_{\infty} \boldsymbol{e}_{z}$ is the incoming momentum at infinity, ${ }^{17}$ as indicated in Fig. 11. We take the orbital angular momentum at infinity to be

$$
\boldsymbol{L}_{\mathrm{in}}=\boldsymbol{b} \times \boldsymbol{p}_{\infty}=b p_{\infty} \boldsymbol{e}_{y},
$$

where $\boldsymbol{b}=-b \boldsymbol{e}_{x}$ and $b$ is the impact parameter. Note that under the conservative dynamics, the spins cannot exchange energy with the remainder of the system because under the equations of motion,

$$
\frac{d\left|\boldsymbol{S}_{a}\right|^{2}}{d t}=2 \boldsymbol{S}_{a} \cdot \dot{\boldsymbol{S}}_{a}=-2 \boldsymbol{S}_{a} \cdot\left(\boldsymbol{S}_{a} \times \frac{\partial H}{\partial \boldsymbol{S}_{a}}\right)=0
$$

\footnotetext{
${ }^{17}$ The notation $p_{\infty}$ is sometimes defined differently, as in Ref. [95].
}

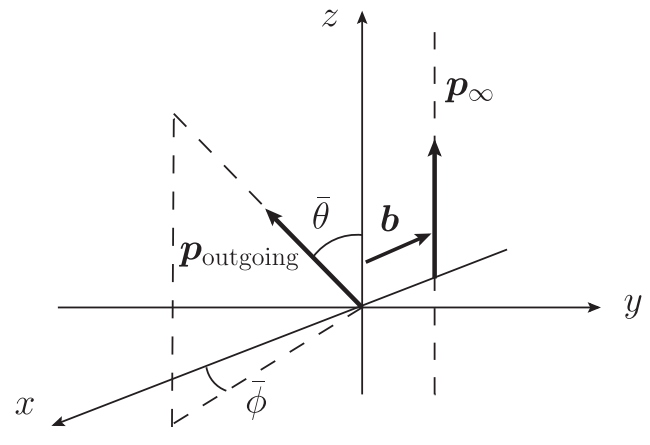

FIG. 11. A representation of a scattering process in the presence of spin. The center of mass is placed at the origin; $\boldsymbol{p}_{\infty}$ is the incoming momentum, $\boldsymbol{p}_{\text {outgoing }}$ is the outgoing momentum, and $\left|\boldsymbol{p}_{\text {outgoing }}\right|=\left|\boldsymbol{p}_{\infty}\right|$. Its direction has been translated to pass through the origin to facilitate the depiction of the two scattering angles $\bar{\theta}$ and $\bar{\phi}$.

where, as in the spin equation of motion (7.2), the index $a=1,2$ is not summed.

We solve the equations of motion perturbatively in Newton's constant; i.e., we search for a solution for coordinates, momenta, and spins of the form

$$
\begin{aligned}
\boldsymbol{r}(t) & =\boldsymbol{r}_{0}(t)+G \boldsymbol{r}_{1}(t)+G^{2} \boldsymbol{r}_{2}(t)+\cdots, \\
\boldsymbol{p}(t) & =\boldsymbol{p}_{0}(t)+G \boldsymbol{p}_{1}(t)+G^{2} \boldsymbol{p}_{2}(t)+\cdots, \\
\boldsymbol{S}_{a}(t) & =\boldsymbol{S}_{a, 0}(t)+G \boldsymbol{S}_{a, 1}(t)+G^{2} \boldsymbol{S}_{a, 2}(t)+\cdots .
\end{aligned}
$$

Replacing them in the equations of motion (7.2) leads to iterative relations between the time derivative of the $n$th term in the expansions above and all the lower-order terms. The $\mathcal{O}\left(G^{0}\right)$ terms describe the motion of a free spinning particle in flat space, i.e., a straight line fixed by the initial momentum, the impact parameter, and the initial spin. The first-order differential equations for the higher-order terms can be integrated; the relevant boundary conditions are that $\boldsymbol{r}_{n \geq 1}, \boldsymbol{p}_{n \geq 1}$, and $\boldsymbol{S}_{a, n \geq 1}$ vanish at $t=-\infty$. The contribution of each order in $G$ to an observable $O$, such as the impulse and spin kick, is then

$\Delta O_{n}=\int_{-\infty}^{\infty} d t \frac{d O_{n}}{d t}=O_{n}(t=+\infty)-O_{n}(t=-\infty)$

with the complete result being their sum weighted with the appropriate powers of $G$.

The incoming and outgoing trajectories approach straight lines at $t= \pm \infty$, respectively, which are along the incoming and outgoing momenta. Thus, the polar and azimuthal scattering angles can be read off in terms of their components or, alternatively, in terms of the incoming momentum and the impulse. Starting with an initial momentum along some generic direction defined by the angles $\theta_{0}$ and $\phi_{0}$, 
$\boldsymbol{p}_{\text {incoming }}=p_{\infty} \sin \theta_{0} \cos \phi_{0} \boldsymbol{e}_{x}+p_{\infty} \sin \theta_{0} \sin \phi_{0} \boldsymbol{e}_{y}$

$$
+p_{\infty} \cos \theta_{0} \boldsymbol{e}_{z}
$$

and then the outgoing momentum, expressed in terms of the scattering angles $\bar{\theta}$ and $\bar{\phi}$, is

$$
\begin{aligned}
\boldsymbol{p}_{\text {outgoing }}= & \boldsymbol{p}_{\text {incoming }}+\Delta \boldsymbol{p} \\
= & p_{\infty} \sin \left(\theta_{0}+\bar{\theta}\right) \cos \left(\phi_{0}+\overline{\boldsymbol{\phi}}\right) \boldsymbol{e}_{x} \\
& +p_{\infty} \sin \left(\theta_{0}+\bar{\theta}\right) \sin \left(\phi_{0}+\bar{\phi}\right) \boldsymbol{e}_{y} \\
& +p_{\infty} \cos \left(\theta_{0}+\bar{\theta}\right) \boldsymbol{e}_{z} .
\end{aligned}
$$

The scattering angles can then easily be extracted in terms of the components of the impulse $\Delta p$ and the incoming momentum. It is worth noting that, if e.g., $\theta_{0}=\pi$ and $\phi_{0}=0$, then the azimuthal angle evaluated at finite Newton's constant exhibits a discontinuity in the limit $G \rightarrow 0$. Indeed, for such a value of $\theta_{0}$, the incoming momentum has vanishing components along $\boldsymbol{e}_{x}$ and $\boldsymbol{e}_{y}$. The components of the outgoing momentum in these directions are both $\mathcal{O}(G)$, leading to $\tan \bar{\phi}=\mathcal{O}(1)$. This discontinuity is unphysical and may easily be remedied by slightly changing the initial conditions such that the incoming momentum is not parallel to a coordinate axis.

\section{B. Impulse, spin kick, and the eikonal phase}

As we will describe in some detail in Ref. [70], by solving Hamilton's equations we find a remarkably simple hidden structure, tying the solution to the gauge-invariant amplitudes. In the case of spinless particles there is a direct link between the physical observables and gauge-invariant quantities extracted from scattering amplitudes $[10,15,39,40]$. Indeed, Refs. [13,38] provide a general formalism for systematically extracting physical observables from amplitudes and their unitarity cuts. For spinning particles such a relation has been found at lowest order in $G$ found in Ref. [38] and further applied in Ref. [61]. Here we show that such relations appear to be general by rewriting the solution for the impulse and spin kick at $\mathcal{O}\left(G^{2}\right)$ in terms of appropriate derivatives acting on the eikonal phase [68], showing that the notion of the eikonal phase naturally generalizes to the case of spin.

As for the spinless case, we define the eikonal phase $\chi=\chi_{1}+\chi_{2}+\cdots$ by Fourier transforms of appropriate parts of amplitudes. The $\mathcal{O}(G)$ contribution to the eikonal phase, in particular, is just the Fourier transform of the tree amplitude to impact parameter space, ${ }^{18}$

$\chi_{1}=\frac{1}{4 m_{1} m_{2} \sqrt{\sigma^{2}-1}} \int \frac{d^{2-2 \epsilon} \boldsymbol{q}}{(2 \pi)^{2-2 \epsilon}} e^{-i \boldsymbol{q} \cdot \boldsymbol{b}} \mathcal{M}^{\mathrm{tree}}(\boldsymbol{q})$,

and the $O\left(G^{2}\right)$ contributions to the eikonal phase is given simply by the triangle contributions to the one-loop amplitudes,

$$
\chi_{2}=\frac{1}{4 m_{1} m_{2} \sqrt{\sigma^{2}-1}} \int \frac{d^{2} \boldsymbol{q}}{(2 \pi)^{2}} e^{-i \boldsymbol{q} \cdot \boldsymbol{b}} \mathcal{M}^{\Delta+\nabla}(\boldsymbol{q}),
$$

where $\mathcal{M}^{\triangle+\nabla}(\boldsymbol{q})$ is given in Eq. (5.43). Since the goal is to compare with results obtained from Hamilton's equations, in the formulas above we must use the amplitudes expressed in terms of the canonical rest-frame spins. The tree-level amplitude in this form is given in Eq. (5.56) while the triangle part of the one-loop amplitude, $\mathcal{M}^{\triangle+\nabla}(\boldsymbol{q})$, is given by the first two lines of Eq. (5.57). The coefficient of each spin-dependent structure is given in Eqs. (5.58), (5.49), and (5.53). Carrying out the Fourier transforms gives the following remarkably compact expressions:

$$
\begin{aligned}
& \chi_{1}=\frac{G \xi E}{|\boldsymbol{p}|}\left[-a_{1}^{(0)} \ln \boldsymbol{b}^{2}-\frac{2 a_{1}^{(1, i)}}{\boldsymbol{b}^{2}}\left(\boldsymbol{p} \times \boldsymbol{S}_{i}\right) \cdot \boldsymbol{b}+a_{1}^{(2,1)}\left(\frac{2}{\boldsymbol{b}^{2}} \boldsymbol{S}_{1 \perp} \cdot \boldsymbol{S}_{2 \perp}-4 \frac{\boldsymbol{S}_{1 \perp} \cdot \boldsymbol{b} \boldsymbol{S}_{2 \perp} \cdot \boldsymbol{b}}{\boldsymbol{b}^{4}}\right)\right], \\
& \chi_{2}=\frac{\pi G^{2} \xi E}{|\boldsymbol{p}|}\left[\frac{a_{2}^{(0)}}{|\boldsymbol{b}|}-\left(a_{2}^{(2,2)} \boldsymbol{S}_{1} \cdot \boldsymbol{S}_{2}+a_{2}^{(2,3)} \boldsymbol{p} \cdot \boldsymbol{S}_{1} \boldsymbol{p} \cdot \boldsymbol{S}_{2}\right) \frac{1}{|\boldsymbol{b}|^{3}}-\frac{a_{2}^{(1, i)}}{|\boldsymbol{b}|^{3}}\left(\boldsymbol{p} \times \boldsymbol{S}_{i}\right) \cdot \boldsymbol{b}+a_{2}^{(2,1)}\left(\frac{1}{|\boldsymbol{b}|^{3}} \boldsymbol{S}_{1 \perp} \cdot \boldsymbol{S}_{2 \perp}-3 \frac{\boldsymbol{S}_{1 \perp} \cdot \boldsymbol{b} \boldsymbol{S}_{2 \perp} \cdot \boldsymbol{b}}{|\boldsymbol{b}|^{5}}\right)\right],
\end{aligned}
$$

where we define

$$
S_{\perp i} \equiv S_{i}-p \frac{S_{i} \cdot p}{p^{2}}
$$

\footnotetext{
${ }^{18}$ At higher orders in $G$ the $\boldsymbol{b}$ in the eikonal formula could differ from the geometric impact parameter [69], but we can set aside this distinction through $\mathcal{O}\left(G^{2}\right)$.
}

Note that the $\boldsymbol{p}$ here, following from the notation in our amplitudes, is the incoming momentum $\boldsymbol{p}_{\infty}$ in Fig. 11, which should not be confused with the intermediate $\boldsymbol{p}$ in Sec. VII A. In Eq. (7.12), we can also use the modelindependent expressions of $a_{i}^{A}$ given in terms of the $c_{i}^{A}$ coefficients appearing in the potential via Eqs. (6.40) and (6.41). Remarkably the simple gauge-invariant functions in Eq. (7.12) encode the physical information for classical scattering processes through $\mathcal{O}\left(G^{2}\right)$, including spinless, 
spin-orbit, and $\operatorname{spin}_{1}$-spin 2 interactions in a form valid to all orders in velocity.

To extract the impulse and spin kick from the eikonal phase, consider the kinematic configuration shown in Fig. 11 where

$$
\begin{aligned}
& \boldsymbol{p}=\left(0,0, p_{\infty}\right), \quad \boldsymbol{b}=(-b, 0,0), \\
& \boldsymbol{L}=\boldsymbol{L}_{\text {in }}=p_{\infty}(0, b, 0) .
\end{aligned}
$$

As mentioned before, $\boldsymbol{p}$ here represents the incoming momentum $\boldsymbol{p}_{\infty}$. By evaluating the eikonal phase on this kinematics and comparing to the solution of the equations of motion, we find that impulse in the $x-y$ plane is [70]

$$
\begin{aligned}
\Delta \boldsymbol{p}_{\perp}= & -\left\{\boldsymbol{P}_{\perp}, \chi\right\}-\frac{1}{2}\left\{\chi,\left\{\boldsymbol{P}_{\perp}, \chi\right\}\right\}-\mathcal{D}_{S L}\left(\chi,\left\{\boldsymbol{P}_{\perp}, \chi\right\}\right) \\
& +\frac{1}{2}\left\{\boldsymbol{P}_{\perp}, \mathcal{D}_{S L}(\chi, \chi)\right\},
\end{aligned}
$$

and the spin kick for all three components is

$$
\begin{aligned}
\Delta \boldsymbol{S}_{i}= & -\left\{\boldsymbol{S}_{i}, \chi\right\}-\frac{1}{2}\left\{\chi,\left\{\boldsymbol{S}_{i}, \chi\right\}\right\}-\mathcal{D}_{S L}\left(\chi,\left\{\boldsymbol{S}_{i}, \chi\right\}\right) \\
& +\frac{1}{2}\left\{\boldsymbol{S}_{i}, \mathcal{D}_{S L}(\chi, \chi)\right\},
\end{aligned}
$$

where both relations are valid up to $\mathcal{O}\left(G^{2}\right)$ and

$$
\begin{aligned}
\left\{\boldsymbol{P}_{\perp}, f\right\} & \equiv-\nabla_{\boldsymbol{b}} f, \\
\left\{S_{a}^{i}, f\right\} & \equiv \epsilon^{i j k} \frac{\partial f}{\partial S_{a}^{j}} S_{a}^{k} \quad(a \text { not summed }), \\
\mathcal{D}_{S L}(f, g) & \equiv-\sum_{a=1,2} \epsilon^{i j k} S_{a}^{k} \frac{\partial f}{\partial S_{a}^{i}} \frac{\partial g}{\partial L^{j}} \\
& =\frac{1}{\boldsymbol{p}^{2}} \sum_{a=1,2}\left(\frac{\partial f}{\partial S_{a}^{j}} \frac{\partial g}{\partial b^{j}} \boldsymbol{S}_{a} \cdot \boldsymbol{p}-p^{j} \frac{\partial f}{\partial S_{a}^{j}} \boldsymbol{S}_{a} \cdot \nabla_{\boldsymbol{b}} g\right),
\end{aligned}
$$

where $f$ and $g$ depend on $\boldsymbol{S}, \boldsymbol{p}$, and $\boldsymbol{b}$. In the brackets $\boldsymbol{p}$ should be taken as inert. To use the first form of $D_{S L}$, we replace $\boldsymbol{b}$ with $\boldsymbol{L}$ via

$$
\boldsymbol{b}=\frac{1}{p^{2}} \boldsymbol{p} \times \boldsymbol{L}
$$

consistent with $\boldsymbol{p} \cdot \boldsymbol{b}=0$. After evaluating the derivatives, we substitute in the values at $t=-\infty$ given in Eq. (7.14) to obtain the impulse and spin kick.

While Eq. (7.15) does not directly give the impulse in the $z$ direction, this quantity follows from energy conservation,

$$
\Delta p_{z}=-\frac{1}{2|\boldsymbol{p}|}(\Delta \boldsymbol{p})^{2},
$$

which can be iteratively solved as a series in $G$. From the structure of the brackets and $D_{S L}$ in Eq. (7.17), the value $S_{a}^{2}$ is preserved, as required from the equation of motion (7.5).

The expressions for the impulse and spin kick in Eqs. (7.15) and (7.16) then match the derived results from the equations of motion, as we have explicitly verified. Together with Eqs. (7.9) and (7.8), they relate the scattering angles and the eikonal phase; in the limit of vanishing spin this relation reproduces the standard one [68], implying the proportionality of the sine of half of the scattering angle and the derivative of the eikonal phase with respect to the absolute value of the impact parameter. An ancillary Mathematica text file [92] contains the explicit values of impulse and spin kick for the initial conditions in Eq. (7.14).

The expressions in Eqs. (7.15) and (7.16) strongly suggest an all orders generalization. For example, one matches the above expressions to the order that they are valid, by

$$
\Delta \mathcal{O}=e^{-i \chi \mathcal{D}}\left[\mathcal{O}, e^{i \chi \mathcal{D}}\right]
$$

where the commutator is related to the brackets in Eq. (7.17) by $[f, g]=i\{f, g\}$ and

$$
\chi \mathcal{D} g \equiv \chi g+i D_{S L}(\chi, g),
$$

for any function $g$ that appears to the right of $\mathcal{D}$. Equation (7.20) is interpreted as being multiplied from the right by a function independent of the orbital angular momentum; alternatively, one may simply define $D_{S L}$ to vanish when it is the rightmost operator in that expression. We defer a detailed discussion of the derivation of these results and their implications to Ref. [70].

\section{CONCLUSIONS}

In this paper we presented a systematic method for constructing the conservative classical Hamiltonian describing the gravitational interaction of two massive spinning bodies. Such Hamiltonians provide crucial input toward obtaining precision gravitational-wave predictions from binary systems that include Kerr black holes or neutron stars. Our formalism extends the arbitrary-spin approach of Refs. [71,72] and incorporates the world-line interactions of Refs. [52,57] into a field-theory framework from which scattering amplitudes can be calculated. The tree-level and one-loop amplitudes we find using this formalism determine the classical two-spinning-body Hamiltonian by EFT matching along the lines of Ref. [12].

We constructed the tree-level amplitude to all orders in spin and velocity and show that it reproduces the $\mathcal{O}(G)$ results of Ref. [58] for the Kerr black hole and extend it to general objects, such as neutron stars with generic spininduced multipole moments. To demonstrate the utility of our approach, we obtained new nontrivial results for the $\operatorname{spin}_{1}-\operatorname{spin}_{2}$ Hamiltonian at $\mathcal{O}\left(G^{2}\right)$ valid to all orders in 
velocity. The bilinear-in-spin part of the one-loop amplitude containing the complete velocity dependence agrees with the spin- $1 / 2$ calculation in Ref. [66]. This is in line with expectations $[23,34,62]$ that this is sufficient at oneloop to capture such spin bilinears. We gave an argument for the lower bound on the value of the spin which is sufficient in our formalism to capture the complete spin dependence of an amplitude at a given loop order.

To extract the two-spinning-body Hamiltonian to all orders in velocity we extended the EFT approach of Ref. [12] to include spin degrees of freedom. In doing so we encountered a subtlety: for the one-loop infrared divergences of the EFT and of the full theory to be the same, it was necessary to have a specific treatment of the terms in the relation between Lorentz generators and spin tensors that are subleading in the classical limit. This procedure guarantees that the constructed EFT corresponds to the relativistic theory we started with. Because of the strongerthan-classical scaling of parts of the loop-level amplitudes, we expect that the matching of infrared divergences must be revisited at every loop order and increasingly more subleading terms be included. Further study is needed to determine whether this procedure is sufficient to fix the subleading terms to all orders in Newton's constant. It may instead be possible to construct a more involved EFT that makes the matching of infrared divergences more straightforward or even avoids it altogether. It would be important to explore both of these strategies toward the obviously interesting problem of systematically constructing the spindependent two-body Hamiltonian at $\mathcal{O}\left(G^{3}\right)$ and beyond.

By suitably choosing the initial conditions, the Hamiltonian derived here can be used to describe any dynamical problem, including the important bound-state cases. For constructing precision gravitational-wave templates that incorporate the new spin information, it is necessary to import these results into models, such as the effective one body approach $[2,16]$.

In this paper we summarized the results of solving the equations of motion for our bilinear-in-spin Hamiltonian in a scattering process and defer a more detailed discussion to Ref. [70]. We obtained the impulse and spin kick in a scattering process. Their construction is substantially more intricate than for the spinless case because orbital angular momentum is not conserved and, consequently, the scattering trajectory is no longer planar. Despite this additional complexity, the results for the impulse and spin kick obtained from the solution to the equations of motion are neatly encoded in the eikonal phase [68,69], obtained by Fourier transforming relevant parts of the amplitude. It is rather striking that the eikonal phase determines the scattering observables, including the spin kick. This points to a much greater hidden simplicity than visible in the Hamiltonian and equations of motion. Based on our results it does seem that a general simple formalism should exist that translates the eikonal phase into generic physical observables. The formalism of Refs. [13,38], which directly expresses physical observables in terms of scattering amplitudes and their unitarity cuts should provide important guidance for further developments along these lines.

We validated our results for the spin-dependent twobody Hamiltonian and the associated observables through several nontrivial checks. Our primary test is that, after expanding in velocity, our result agrees with the state-ofthe-art calculations of spin-orbit and $\operatorname{spin}_{1}-\operatorname{spin}_{2}$ potentials in the post-Newtonian framework [54,55] in the overlapped region. Truncating our Hamiltonian to spin-orbit interactions, we also reproduce the all-orders-in-velocity scattering angle obtained in Ref. [59], whose spin-orbit part is in agreement with Ref. [64], for the configuration where the spins of the two bodies are aligned with the orbital angular momentum. An additional nontrivial test is in the test-mass limit, in which we reproduce the all-orders-in-velocity results of Refs. [75]. (See also Refs. [65,91].)

While the Lagrangian for higher-spin fields we constructed here is not directly suitable for quantum loop calculations with internal higher-spin fields, it is sufficient for tree-level calculations, which in turn are sufficient for constructing all unitarity cuts required in the classical limit. A very interesting direction that can usefully impact the complexity of constructing higher order spin-dependent Hamiltonians is to systematically expand our understanding of the double copy including spin. The double copy expresses gravitational amplitudes in terms of simpler gauge-theory ones. Here we pointed out some tantalizing double-copy relations. This includes double-copy properties of the two-matter-one-graviton tree-level vertices, corresponding to the energy-momentum tensor for arbitrary spin [58]. In addition, we presented a KLT-like factorization for the tree-level gravitational Compton amplitude.

An obvious problem is to extend the results obtained here to high powers of spin at $\mathcal{O}\left(G^{2}\right)$ and beyond. As we have argued, amplitudes of low-spin particles are in general insufficient for this purpose because of special relations between Lorentz generators in fixed representations. We set up our arbitrary-spin formalism precisely to avoid these limitations. The higher-spin Lagrangian we used captures the covariantization of the parity-even spin-induced gravitational linear response functions and thus includes all parity-even multipole moments. As the number of spin operators increases, nonlinear response functions, described by operators with two or more gravitons, also need to be included. Here we avoided them by focusing on terms that, while bilinear in spins, are at most linear in the spin of each particle. An important problem is the complete classification of all such operators (each containing as many Riemann tensors as the desired number of gravitons) and the evaluation of their contribution to the effective Hamiltonian of massive spinning bodies. For black holes, it may be possible to fix coefficients through purely theoretical considerations as done for the energy-momentum 
tensor [58]. For neutron stars or other astrophysical objects, the coefficients carry information about its internal structure and properties, and should be treated as phenomenological parameters, to be determined by observation. The first contribution of such an operator to the two-body Hamiltonian depends on the number of gravitons it contains. For example, the two-graviton operators first contribute at $\mathcal{O}\left(G^{2}\right)$ to conservative processes and at $\mathcal{O}\left(G^{3 / 2}\right)$ to processes with outgoing gravitational radiation.

It would also be of crucial importance to see what further progress can be made in developing an eikonal formalism that includes arbitrary spin contributions at any order and to understand in detail the extent of the direct links between finite parts of scattering amplitudes and physical quantities. It would also be important to see whether appropriate analytic continuations can relate observables of the unbound and bound motion, as for the spin-aligned case [39].

In summary, we expect the amplitude-based effectivefield-theory approach advocated here to lead to further progress on the spin dependence of gravitational interactions. Our linkage of scattering observables to the eikonal phase demonstrates a surprising hidden simplicity which suggests that better methods for constructing physical observables may exist. This will be further discussed in Ref. [70].

\section{ACKNOWLEDGMENTS}

We especially thank Alessandra Buonanno, Jan Steinhoff, and Justin Vines for a number of very helpful discussions and for supplying a simpler form of the testmass Hamitonian based on Ref. [75]. We also thank Clifford Cheung, Thibault Damour, David Kosower, Aneesh Manohar, Donal O'Connell, Julio Parra-Marinez, Rafael Porto, Ira Rothstein, and Mikhail Solon for many helpful discussions. Z. B. and A. L. are supported by the U.S. Department of Energy (DOE) under Award No. DESC0009937. R. R. is supported by the U.S. Department of Energy (DOE) under Grant No. DE-SC0013699. C. H. S. is supported by the Mani L. Bhaumik Institute for Theoretical Physics. M. Z. is supported by the Swiss National Science Foundation under Contract No. SNF200021 179016 and the European Commission through the ERC Grant pertQCD.
[1] B. P. Abbott et al. (LIGO Scientific and Virgo Collaborations), Observation of Gravitational Waves from a Binary Black Hole Merger, Phys. Rev. Lett. 116, 061102 (2016); GW170817: Observation of Gravitational Waves from a Binary Neutron Star Inspiral, Phys. Rev. Lett. 119, 161101 (2017).

[2] A. Buonanno and T. Damour, Effective one-body approach to general relativistic two-body dynamics, Phys. Rev. D 59, 084006 (1999); Transition from inspiral to plunge in binary black hole coalescences, Phys. Rev. D 62, 064015 (2000).

[3] F. Pretorius, Evolution of Binary Black Hole Spacetimes, Phys. Rev. Lett. 95, 121101 (2005); M. Campanelli, C. O. Lousto, P. Marronetti, and Y. Zlochower, Accurate Evolutions of Orbiting Black-Hole Binaries Without Excision, Phys. Rev. Lett. 96, 111101 (2006); J. G. Baker, J. Centrella, D. I. Choi, M. Koppitz, and J. van Meter, Gravitational Wave Extraction from an Inspiraling Configuration of Merging Black Holes, Phys. Rev. Lett. 96, 111102 (2006).

[4] Y. Mino, M. Sasaki, and T. Tanaka, Gravitational radiation reaction to a particle motion, Phys. Rev. D 55, 3457 (1997); T. C. Quinn and R. M. Wald, An Axiomatic approach to electromagnetic and gravitational radiation reaction of particles in curved space-time, Phys. Rev. D 56, 3381 (1997).

[5] J. Droste, The field of $n$ moving centres in Einstein's theory of gravitation, Proc. Acad. Sci. Amsterdam 19, 447 (1916); H. A. Lorentz and J. Droste, De beweging van een stelsel lichamen onder de theorie van Einstein I,II Koninklijke Akademie Van Wetenschappen te Amsterdam 26 392, 649 (1917) [English translation in Lorentz Collected papers, in
The Hague: Nijhof, edited by (1934-1939), Vol. 5, p. 330]; A. Einstein, L. Infeld, and B. Hoffmann, The gravitational equations and the problem of motion, Ann. Math. 39, 65 (1938); T. Ohta, H. Okamura, T. Kimura, and K. Hiida, Physically acceptable solution of einstein's equation for many-body system, Prog. Theor. Phys. 50, 492 (1973); P. Jaranowski and G. Schäfer, Third post-Newtonian higher order ADM Hamilton dynamics for two-body point mass systems, Phys. Rev. D 57, 7274 (1998); , Erratum, Phys. Rev. D 63, 029902 (2000); T. Damour, P. Jaranowski, and G. Schäfer, Dynamical invariants for general relativistic two-body systems at the third post-Newtonian approximation, Phys. Rev. D 62, 044024 (2000); L. Blanchet and G. Faye, Equations of motion of point particle binaries at the third post-Newtonian order, Phys. Lett. A 271, 58 (2000); T. Damour, P. Jaranowski, and G. Schäfer, Dimensional regularization of the gravitational interaction of point masses, Phys. Lett. B 513, 147 (2001); T. Damour, P. Jaranowski, and G. Schäfer, Nonlocal-in-time action for the fourth post-Newtonian conservative dynamics of two-body systems, Phys. Rev. D 89, 064058 (2014).

[6] P. Jaranowski and G. Schäfer, Derivation of local-in-time fourth post-Newtonian ADM Hamiltonian for spinless compact binaries, Phys. Rev. D 92, 124043 (2015).

[7] W. D. Goldberger and I.Z. Rothstein, An effective field theory of gravity for extended objects, Phys. Rev. D 73, 104029 (2006).

[8] B. Kol and M. Smolkin, Classical effective field theory and caged black holes, Phys. Rev. D 77, 064033 (2008); B. Kol and M. Smolkin, Non-relativistic gravitation: From Newton 
to Einstein and back, Classical Quantum Gravity 25, 145011 (2008); J. B. Gilmore and A. Ross, Effective field theory calculation of second post-Newtonian binary dynamics, Phys. Rev. D 78, 124021 (2008); S. Foffa and R. Sturani, Effective field theory calculation of conservative binary dynamics at third post-Newtonian order, Phys. Rev. D 84, 044031 (2011); S. Foffa, P. Mastrolia, R. Sturani, and C. Sturm, Effective field theory approach to the gravitational two-body dynamics, at fourth post-Newtonian order and quintic in the Newton constant, Phys. Rev. D 95, 104009 (2017); R. A. Porto and I. Z. Rothstein, Apparent ambiguities in the post-Newtonian expansion for binary systems, Phys. Rev. D 96, 024062 (2017); S. Foffa, P. Mastrolia, R. Sturani, C. Sturm, and W. J. T. Bobadilla, Static Two-Body Potential at Fifth Post-Newtonian Order, Phys. Rev. Lett. 122, 241605 (2019); J. Blümlein, A. Maier, and P. Marquard, Five-Loop static contribution to the gravitational interaction potential of two point masses, Phys. Lett. B 800, 135100 (2020); S. Foffa and R. Sturani, Conservative dynamics of binary systems to fourth Post-Newtonian order in the EFT approach I: Regularized Lagrangian, Phys. Rev. D 100, 024047 (2019); S. Foffa, R. A. Porto, I. Rothstein, and R. Sturani, Conservative dynamics of binary systems to fourth Post-Newtonian order in the EFT approach II: Renormalized Lagrangian, Phys. Rev. D 100, 024048 (2019); J. Blümlein, A. Maier, and P. Marquard, Five-loop static contribution to the gravitational interaction potential of two point masses, Phys. Lett. B 800, 135100 (2020); J. Blümlein, A. Maier, P. Marquard, and G. Schäfer, Fourth post-Newtonian Hamiltonian dynamics of two-body systems from an effective field theory approach, arXiv: 2003.01692.

[9] B. Bertotti, On gravitational motion, Nuovo Cimento 4, 898 (1956); R. P. Kerr, The Lorentz-covariant approximation method in general relativity, Nuovo Cimento (1955-1965) 13, 469 (1959); B. Bertotti and J. F. Plebański, Theory of gravitational perturbations in the fast motion approximation, Ann. Phys. (N.Y.) 11, 169 (1960); M. Portilla, Momentum and angular momentum of two gravitating particles, J. Phys. A 12, 1075 (1979); K. Westpfahl and M. Goller, Gravitational scattering of two relativistic particles in postlinear approximation, Lett. Nuovo Cimento 26, 573 (1979); M. Portilla, Scattering of two gravitating particles: Classical approach, J. Phys. A 13, 3677 (1980); L. Bel, T. Damour, N. Deruelle, J. Ibanez, and J. Martin, Poincaré-invariant gravitational field and equations of motion of two pointlike objects: The postlinear approximation of general relativity, Gen. Relativ. Gravit. 13, 963 (1981); K. Westpfahl, Highspeed scattering of charged and uncharged particles in general relativity, Fortschr. Phys. 33, 417 (1985); T. Ledvinka, G. Schaefer, and J. Bicak, Relativistic ClosedForm Hamiltonian for Many-Body Gravitating Systems in the Post-Minkowskian Approximation, Phys. Rev. Lett. 100, 251101 (2008).

[10] T. Damour, High-energy gravitational scattering and the general relativistic two-body problem, Phys. Rev. D 97, 044038 (2018).

[11] N. E. J. Bjerrum-Bohr, J. F. Donoghue, and P. Vanhove, Onshell techniques and universal results in quantum gravity, J. High Energy Phys. 02 (2014) 111; N. E. J. Bjerrum-Bohr,
P. H. Damgaard, G. Festuccia, L. Planté, and P. Vanhove, General Relativity from Scattering Amplitudes, Phys. Rev. Lett. 121, 171601 (2018).

[12] C. Cheung, I. Z. Rothstein, and M. P. Solon, From Scattering Amplitudes to Classical Potentials in the Post-Minkowskian Expansion, Phys. Rev. Lett. 121, 251101 (2018).

[13] D. A. Kosower, B. Maybee, and D. O'Connell, Amplitudes, observables, and classical scattering, J. High Energy Phys. 02 (2019) 137.

[14] Z. Bern, C. Cheung, R. Roiban, C. H. Shen, M. P. Solon, and M. Zeng, Scattering Amplitudes and the Conservative Hamiltonian for Binary Systems at Third Post-Minkowskian Order, Phys. Rev. Lett. 122, 201603 (2019).

[15] Z. Bern, C. Cheung, R. Roiban, C. H. Shen, M. P. Solon, and M. Zeng, Black hole binary dynamics from the double copy and effective theory, J. High Energy Phys. 10 (2019) 206.

[16] A. Antonelli, A. Buonanno, J. Steinhoff, M. van de Meent, and J. Vines, Energetics of two-body Hamiltonians in postMinkowskian gravity, Phys. Rev. D 99, 104004 (2019).

[17] A. K. Collado, P. Di Vecchia, and R. Russo, Revisiting the second post-Minkowskian eikonal and the dynamics of binary black holes, Phys. Rev. D 100, 066028 (2019); A. Cristofoli, P. H. Damgaard, P. Di Vecchia, and C. Heissenberg, Second-order Post-Minkowskian scattering in arbitrary dimensions, J. High Energy Phys. 07 (2020) 122.

[18] J. Blümlein, A. Maier, P. Marquard, and G. Schäfer, Testing binary dynamics in gravity at the sixth post-Newtonian level, Phys. Lett. B 807, 135496 (2020); C. Cheung and M. P. Solon, Classical gravitational scattering at $\mathcal{O}\left(G^{3}\right)$ from Feynman diagrams, J. High Energy Phys. 06 (2020) 144; D. Bini, T. Damour, and A. Geralico, Binary dynamics at the fifth and fifth-and-a-half post-Newtonian orders, Phys. Rev. D 102, 024062 (2020); Sixth post-Newtonian local-in-time dynamics of binary systems, Phys. Rev. D 102, 024061 (2020).

[19] D. Bini, T. Damour, and A. Geralico, Novel Approach to Binary Dynamics: Application to the Fifth Post-Newtonian Level, Phys. Rev. Lett. 123, 231104 (2019); N. Siemonsen and J. Vines, Test black holes, scattering amplitudes and perturbations of Kerr spacetime, arXiv:1909.07361; A. Antonelli, C. Kavanagh, M. Khalil, J. Steinhoff, and J. Vines, Gravitational spin-orbit coupling through third-subleading post-Newtonian order: From first-order self-force to arbitrary mass ratios, arXiv:2003.11391.

[20] L. Blanchet, Gravitational radiation from post-Newtonian sources and inspiralling compact binaries, Living Rev. Relativity 17, 2 (2014); G. Schäfer and P. Jaranowski, Hamiltonian formulation of general relativity and postNewtonian dynamics of compact binaries, Living Rev. Relativity 21, 7 (2018); L. Barack and A. Pound, Self-force and radiation reaction in general relativity, Rept. Prog. Phys. 82, 016904 (2019); L. Barack et al., Black holes, gravitational waves and fundamental physics: A roadmap, Classical Quantum Gravity 36, 143001 (2019); M. Levi, Effective field theories of post-Newtonian gravity: A comprehensive review, arXiv:1807.01699.

[21] R. A. Porto, The effective field theorist's approach to gravitational dynamics, Phys. Rep. 633, 1 (2016). 
[22] D. Neill and I. Z. Rothstein, Classical space-times from the S matrix, Nucl. Phys. B877, 177 (2013).

[23] V. Vaidya, Gravitational spin Hamiltonians from the S matrix, Phys. Rev. D 91, 024017 (2015).

[24] M. L. Mangano and S. J. Parke, Multiparton amplitudes in gauge theories, Phys. Rep. 200, 301 (1991); L. J. Dixon, Calculating scattering amplitudes efficiently, arXiv:hep-ph/ 9601359; Z. Bern, L. J. Dixon, and D. A. Kosower, Progress in one loop QCD computations, Annu. Rev. Nucl. Part. Sci. 46, 109 (1996); H. Elvang and Y.t. Huang, Scattering amplitudes, arXiv:1308.1697.

[25] Z. Bern, J. J. Carrasco, M. Chiodaroli, H. Johansson, and R. Roiban, The duality between color and kinematics and its applications, arXiv:1909.01358.

[26] R. Britto, F. Cachazo, B. Feng, and E. Witten, Direct Proof of Tree-Level Recursion Relation in Yang-Mills Theory, Phys. Rev. Lett. 94, 181602 (2005).

[27] Z. Bern, L. J. Dixon, D. C. Dunbar, and D. A. Kosower, One loop $n$-point gauge-theory amplitudes, unitarity and collinear limits, Nucl. Phys. B425, 217 (1994); Z. Bern, L. J. Dixon, D. C. Dunbar, and D. A. Kosower, Fusing gaugetheory tree amplitudes into loop amplitudes, Nucl. Phys. B435, 59 (1995); Z. Bern, L. J. Dixon, and D. A. Kosower, One loop amplitudes for $e^{+} e^{-}$to four partons, Nucl. Phys. B513, 3 (1998); Z. Bern, L. J. Dixon, D. C. Dunbar, M. Perelstein, and J. S. Rozowsky, On the relationship between Yang-Mills theory and gravity and its implication for ultraviolet divergences, Nucl. Phys. B530, 401 (1998); R. Britto, F. Cachazo, and B. Feng, Generalized unitarity and one-loop amplitudes in $N=4$ super-Yang-Mills, Nucl. Phys. B725, 275 (2005); Z. Bern, J. J. M. Carrasco, H. Johansson, and D. A. Kosower, Maximally supersymmetric planar Yang-Mills amplitudes at five loops, Phys. Rev. D 76, 125020 (2007).

[28] Z. Bern and A. G. Morgan, Massive loop amplitudes from unitarity, Nucl. Phys. B467, 479 (1996).

[29] Z. Bern, L. J. Dixon, and D. A. Kosower, Progress in one loop QCD computations, Annu. Rev. Nucl. Part. Sci. 46, 109 (1996); Z. Bern and Y. t. Huang, Basics of generalized unitarity, J. Phys. A 44, 454003 (2011); J. J. M. Carrasco and H. Johansson, Generic multiloop methods and application to $N=4$ super-Yang-Mills, J. Phys. A 44, 454004 (2011).

[30] G. Ossola, C. G. Papadopoulos, and R. Pittau, Reducing full one-loop amplitudes to scalar integrals at the integrand level, Nucl. Phys. B763, 147 (2007).

[31] D. Forde, Direct extraction of one-loop integral coefficients, Phys. Rev. D 75, 125019 (2007).

[32] Y. Iwasaki, Quantum theory of gravitation vs. classical theory-fourth-order potential, Prog. Theor. Phys. 46, 1587 (1971); Y. Iwasaki, Fourth-order gravitational potential based on quantum field theory, Lett. Nuovo Cimento 1, 783 (1971); 1, 783 (1971); S. N. Gupta and S. F. Radford, Improved gravitational coupling of scalar fields, Phys. Rev. D 19, 1065 (1979).

[33] J. F. Donoghue, General relativity as an effective field theory: The leading quantum corrections, Phys. Rev. D 50, 3874 (1994); B. R. Holstein and J. F. Donoghue, Classical Physics and Quantum Loops, Phys. Rev. Lett. 93, 201602 (2004).
[34] B. R. Holstein and A. Ross, Spin effects in long range gravitational scattering, arXiv:0802.0716.

[35] T. Damour, Gravitational scattering, post-Minkowskian approximation and effective one-body theory, Phys. Rev. D 94, 104015 (2016); A. Cristofoli, N. E. J. Bjerrum-Bohr, P. H. Damgaard, and P. Vanhove, On post-Minkowskian Hamiltonians in general relativity, arXiv:1906.01579.

[36] F. Cachazo and A. Guevara, Leading singularities and classical gravitational scattering, J. High Energy Phys. 02 (2020) 181.

[37] A. Guevara, Holomorphic classical limit for spin effects in gravitational and electromagnetic scattering, J. High Energy Phys. 04 (2019) 033.

[38] B. Maybee, D. O'Connell, and J. Vines, Observables and amplitudes for spinning particles and black holes, J. High Energy Phys. 12 (2019) 156.

[39] G. Kälin and R. A. Porto, From boundary data to bound states, J. High Energy Phys. 01 (2020) 072; From boundary data to bound states II: Scattering angle to dynamical invariants (with twist), J. High Energy Phys. 02 (2020) 120 .

[40] N. E. J. Bjerrum-Bohr, A. Cristofoli, and P. H. Damgaard, Post-Minkowskian scattering angle in Einstein gravity, J. High Energy Phys. 08 (2020) 038.

[41] H. Kawai, D. C. Lewellen, and S. H. H. Tye, A relation between tree amplitudes of closed and open strings, Nucl. Phys. B269, 1 (1986); Z. Bern, L. J. Dixon, M. Perelstein, and J. S. Rozowsky, Multileg one loop gravity amplitudes from gauge theory, Nucl. Phys. B546, 423 (1999).

[42] Z. Bern, J. J. M. Carrasco, and H. Johansson, New relations for gauge-theory amplitudes, Phys. Rev. D 78, 085011 (2008).

[43] Z. Bern, J. J. M. Carrasco, and H. Johansson, Perturbative Quantum Gravity as a Double Copy of Gauge Theory, Phys. Rev. Lett. 105, 061602 (2010).

[44] Z. Bern, J. J. Carrasco, L. J. Dixon, H. Johansson, and R. Roiban, The Ultraviolet Behavior of $N=8$ Supergravity at Four Loops, Phys. Rev. Lett. 103, 081301 (2009); Z. Bern, J. J. M. Carrasco, L. J. Dixon, H. Johansson, and R. Roiban, Simplifying multiloop integrands and ultraviolet divergences of gauge theory and gravity amplitudes, Phys. Rev. D 85, 105014 (2012); Z. Bern, S. Davies, T. Dennen, A. V. Smirnov, and V.A. Smirnov, Ultraviolet Properties of $N=4$ Supergravity at Four Loops, Phys. Rev. Lett. 111, 231302 (2013); Z. Bern, S. Davies, and T. Dennen, Enhanced ultraviolet cancellations in $\mathcal{N}=5$ supergravity at four loops, Phys. Rev. D 90, 105011 (2014); Z. Bern, J. J. M. Carrasco, W. M. Chen, H. Johansson, R. Roiban, and M. Zeng, Five-loop four-point integrand of $N=8$ supergravity as a generalized double copy, Phys. Rev. D 96, 126012 (2017); Z. Bern, J. J. Carrasco, W. M. Chen, A. Edison, H. Johansson, J. Parra-Martinez, R. Roiban, and M. Zeng, Ultraviolet properties of $\mathcal{N}=8$ supergravity at five loops, Phys. Rev. D 98, 086021 (2018).

[45] Z. Bern, C. Cheung, H. H. Chi, S. Davies, L. Dixon, and J. Nohle, Evanescent Effects Can Alter Ultraviolet Divergences in Quantum Gravity Without Physical Consequences, Phys. Rev. Lett. 115, 211301 (2015); Z. Bern, H. H. Chi, L. Dixon, and A. Edison, Two-loop renormalization of quantum gravity simplified, Phys. Rev. D 95, 046013 (2017). 
[46] F. A. Berends, R. Kleiss, P. De Causmaecker, R. Gastmans, and T. T. Wu, Single bremsstrahlung processes in gauge theories, Phys. Lett. 103B, 124 (1981); F. A. Berends, R. Kleiss, P. De Causmaecker, R. Gastmans, W. Troost, and T. T. Wu, Multiple bremsstrahlung in gauge theories at highenergies. 2. single bremsstrahlung, Nucl. Phys. B206, 61 (1982); Z. Xu, D. H. Zhang, and L. Chang, Helicity amplitudes for multiple bremsstrahlung in massless nonabelian gauge theories, Nucl. Phys. B291, 392 (1987).

[47] N. Arkani-Hamed, T. C. Huang, and Y. t. Huang, Scattering amplitudes for all masses and spins, arXiv:1709.04891.

[48] B. P. Abbott et al. (LIGO Scientific and Virgo Collaborations), GW151226: Observation of Gravitational Waves from a 22-Solar-Mass Binary Black Hole Coalescence, Phys. Rev. Lett. 116, 241103 (2016); GW190412: Observation of a binary-black-hole coalescence with asymmetric masses, arXiv:2004.08342.

[49] M. A. Abramowicz and W. Kluzniak, A precise determination of angular momentum in the black hole candidate GRO J1655-40, Astron. Astrophys. 374, L19 (2001); A. C. Fabian and G. Miniutti, The X-ray spectra of accreting Kerr black holes, arXiv:astro-ph/0507409; J. E. McClintock, R. Shafee, R. Narayan, R. A. Remillard, S. W. Davis, and L. X. $\mathrm{Li}$, The Spin of the near-extreme Kerr black hole GRS $1915+105$, Astrophys. J. 652, 518 (2006); L. W. Brenneman and C. S. Reynolds, Constraining black hole spin via X-ray spectroscopy, Astrophys. J. 652, 1028 (2006); L. W. Brenneman et al., The spin of the supermassive black hole in NGC 3783, Astrophys. J. 736, 103 (2011); L. Gou et al., The extreme spin of the black hole in Cygnus X-1, Astrophys. J. 742, 85 (2011); M. A. Nowak, J. Wilms, K. Pottschmidt, N. Schulz, D. Maitra, and J. Miller, Suzaku observations of $4 \mathrm{U} 1957+11$ : Potentially the most rapidly spinning black hole in (the Halo of) the galaxy, Astrophys. J. 744, 107 (2012); C. S. Reynolds, Measuring black hole spin using x-ray reflection spectroscopy, Space Sci. Rev. 183, 277 (2014).

[50] C. Cutler and E. E. Flanagan, Gravitational waves from merging compact binaries: How accurately can one extract the binary's parameters from the inspiral wave form?, Phys. Rev. D 49, 2658 (1994); T. A. Apostolatos, C. Cutler, G. J. Sussman, and K. S. Thorne, Spin induced orbital precession and its modulation of the gravitational wave forms from merging binaries, Phys. Rev. D 49, 6274 (1994).

[51] B. Barker and R. O'Connell, Derivation of the equations of motion of a gyroscope from the quantum theory of gravitation, Phys. Rev. D 2, 1428 (1970); B. Barker and R. O'Connell, Gravitational two-body problem with arbitrary masses, spins, and quadrupole moments, Phys. Rev. D 12, 329 (1975); L. E. Kidder, C. M. Will, and A. G. Wiseman, Spin effects in the inspiral of coalescing compact binaries, Phys. Rev. D 47, R4183 (1993); L. E. Kidder, Coalescing binary systems of compact objects to postNewtonian $5 / 2$ order. 5. Spin effects, Phys. Rev. D 52, 821 (1995); H. Tagoshi, A. Ohashi, and B. J. Owen, Gravitational field and equations of motion of spinning compact binaries to 2.5 postNewtonian order, Phys. Rev. D 63, 044006 (2001); G. Faye, L. Blanchet, and A. Buonanno, Higher-order spin effects in the dynamics of compact binaries. I. Equations of motion, Phys. Rev. D 74, 104033 (2006); L. Blanchet,
A. Buonanno, and G. Faye, Higher-order spin effects in the dynamics of compact binaries. II. Radiation field, Phys. Rev. D 74, 104034 (2006); , Erratum, Phys. Rev. D 75, 049903 (2007); , Erratum, Phys. Rev. D 81, 089901 (2010); T. Damour, P. Jaranowski, and G. Schaefer, Hamiltonian of two spinning compact bodies with next-to-leading order gravitational spin-orbit coupling, Phys. Rev. D 77, 064032 (2008); J. Steinhoff, S. Hergt, and G. Schaefer, On the nextto-leading order gravitational spin(1)-spin(2) dynamics, Phys. Rev. D 77, 081501 (2008); J. Steinhoff, S. Hergt, and G. Schaefer, Spin-squared Hamiltonian of next-toleading order gravitational interaction, Phys. Rev. D 78, 101503 (2008); S. Hergt, J. Steinhoff, and G. Schaefer, Reduced Hamiltonian for next-to-leading order spinsquared dynamics of general compact binaries, Classical Quantum Gravity 27, 135007 (2010); S. Marsat, L. Blanchet, A. Bohe, and G. Faye, Gravitational waves from spinning compact object binaries: New post-Newtonian results, arXiv:1312.5375; S. Hergt, J. Steinhoff, and G. Schaefer, On the comparison of results regarding the post-Newtonian approximate treatment of the dynamics of extended spinning compact binaries, J. Phys. Conf. Ser. 484, 012018 (2014); S. Marsat, A. Bohe, G. Faye, and L. Blanchet, Nextto-next-to-leading order spin-orbit effects in the equations of motion of compact binary systems, Classical Quantum Gravity 30, 055007 (2013); A. Bohe, S. Marsat, G. Faye, and L. Blanchet, Next-to-next-to-leading order spin-orbit effects in the near-zone metric and precession equations of compact binaries, Classical Quantum Gravity 30, 075017 (2013); A. Bohé, G. Faye, S. Marsat, and E. K. Porter, Quadratic-in-spin effects in the orbital dynamics and gravitational-wave energy flux of compact binaries at the 3PN order, Classical Quantum Gravity 32, 195010 (2015); D. Bini, A. Geralico, and J. Vines, Hyperbolic scattering of spinning particles by a Kerr black hole, Phys. Rev. D 96, 084044 (2017); N. Siemonsen, J. Steinhoff, and J. Vines, Gravitational waves from spinning binary black holes at the leading post-Newtonian orders at all orders in spin, Phys. Rev. D 97, 124046 (2018).

[52] R. A. Porto, Post-Newtonian corrections to the motion of spinning bodies in NRGR, Phys. Rev. D 73, 104031 (2006); R. A. Porto and I. Z. Rothstein, Next to leading order spin(1)spin(1) effects in the motion of inspiralling compact binaries, Phys. Rev. D 78, 044013 (2008); , Erratum, Phys. Rev. D 81, 029905 (2010).

[53] R. A. Porto and I. Z. Rothstein, The Hyperfine EinsteinInfeld-Hoffmann Potential, Phys. Rev. Lett. 97, 021101 (2006); R. A. Porto and I. Z. Rothstein, Comment on 'On the next-to-leading order gravitational $\operatorname{spin}(1)-\operatorname{spin}(2)$ dynamics' by J. Steinhoff et al., arXiv:0712.2032; R. A. Porto and I.Z. Rothstein, Spin(1)Spin(2) effects in the motion of inspiralling compact binaries at third order in the post-Newtonian expansion, Phys. Rev. D 78, 044012 (2008); Erratum, Phys. Rev. D 81, 029904 (2010); M. Levi, Next to Leading Order gravitational Spin1-Spin2 coupling with Kaluza-Klein reduction, Phys. Rev. D 82, 064029 (2010); R. A. Porto, Next to leading order spin-orbit effects in the motion of inspiralling compact binaries, Classical Quantum Gravity 27, 205001 (2010); M. Levi, next to leading order gravitational spin-orbit coupling in an 
effective field theory approach, Phys. Rev. D 82, 104004 (2010); M. Levi, Binary dynamics from spin1-spin2 coupling at fourth post-Newtonian order, Phys. Rev. D 85, 064043 (2012); M. Levi and J. Steinhoff, Next-to-next-toleading order gravitational spin-orbit coupling via the effective field theory for spinning objects in the postNewtonian scheme, J. Cosmol. Astropart. Phys. 01 (2016) 011; M. Levi and J. Steinhoff, Next-to-next-toleading order gravitational spin-squared potential via the effective field theory for spinning objects in the postNewtonian scheme, J. Cosmol. Astropart. Phys. 01 (2016) 008; M. Levi and J. Steinhoff, Complete conservative dynamics for inspiralling compact binaries with spins at fourth post-Newtonian order, arXiv: 1607.04252; R. A. Porto, A. Ross, and I. Z. Rothstein, Spin induced multipole moments for the gravitational wave flux from binary inspirals to third Post-Newtonian order, J. Cosmol. Astropart. Phys. 03 (2011) 009; Spin induced multipole moments for the gravitational wave amplitude from binary inspirals to 2.5 Post-Newtonian order, J. Cosmol. Astropart. Phys. 09 (2012) 028; N. T. Maia, C. R. Galley, A. K. Leibovich, and R. A. Porto, Radiation reaction for spinning bodies in effective field theory I: Spinorbit effects, Phys. Rev. D 96, 084064 (2017); M. Levi, S. Mougiakakos, and M. Vieira, Gravitational cubic-in-spin interaction at the next-to-leading post-Newtonian order, arXiv:1912.06276; M. Levi, A. J. Mcleod, and M. Von Hippel, $\mathrm{N}^{3} \mathrm{LO}$ gravitational spin-orbit coupling at order $G^{4}$, arXiv:2003.02827; NNNLO gravitational quadratic-in-spin interactions at the quartic order in G, arXiv:2003.07890.

[54] J. Steinhoff, G. Schaefer, and S. Hergt, ADM canonical formalism for gravitating spinning objects, Phys. Rev. D 77, 104018 (2008).

[55] J. Hartung, J. Steinhoff, and G. Schafer, Next-to-next-toleading order post-Newtonian linear-in-spin binary Hamiltonians, Ann. Phys. (Berlin) 525, 359 (2013).

[56] M. Levi and J. Steinhoff, Equivalence of ADM Hamiltonian and effective field theory approaches at next-to-next-toleading order spin1-spin2 coupling of binary inspirals, J. Cosmol. Astropart. Phys. 12 (2014) 003.

[57] M. Levi and J. Steinhoff, Spinning gravitating objects in the effective field theory in the post-Newtonian scheme, J. High Energy Phys. 09 (2015) 219.

[58] J. Vines, Scattering of two spinning black holes in postMinkowskian gravity, to all orders in spin, and effectiveone-body mappings, Classical Quantum Gravity 35, 084002 (2018).

[59] A. Guevara, A. Ochirov, and J. Vines, Scattering of Spinning Black Holes from Exponentiated Soft Factors, J. High Energy Phys. 09 (2019) 056.

[60] M. Z. Chung, Y. T. Huang, J. W. Kim, and S. Lee, The simplest massive S-matrix: From minimal coupling to Black Holes, J. High Energy Phys. 04 (2019) 156.

[61] A. Guevara, A. Ochirov, and J. Vines, Black-hole scattering with general spin directions from minimal-coupling amplitudes, Phys. Rev. D 100, 104024 (2019).

[62] M. Z. Chung, Y. T. Huang, and J. W. Kim, From quantized spins to rotating black holes, J. High Energy Phys. 09 (2020) 074.
[63] M. Z. Chung, Y.t. Huang, J. W. Kim, and S. Lee, Complete Hamiltonian for spinning binary systems at first post-Minkowskian order, J. High Energy Phys. 05 (2020) 105.

[64] D. Bini and T. Damour, Gravitational spin-orbit coupling in binary systems, post-Minkowskian approximation and effective one-body theory, Phys. Rev. D 96, 104038 (2017); Gravitational spin-orbit coupling in binary systems at the second post-Minkowskian approximation, Phys. Rev. D 98, 044036 (2018).

[65] J. Vines, J. Steinhoff, and A. Buonanno, Spinning-blackhole scattering and the test-black-hole limit at second postMinkowskian order, Phys. Rev. D 99, 064054 (2019).

[66] P. H. Damgaard, K. Haddad, and A. Helset, Heavy black hole effective theory, J. High Energy Phys. 11 (2019) 070.

[67] R. Aoude, K. Haddad, and A. Helset, On-shell heavy particle effective theories, J. High Energy Phys. 05 (2020) 051.

[68] R. J. Glauber, in Lectures in Theoretical Physics, edited by W. E. Brittin and L. G. Dunham (Interscience Publishers, Inc., New York, 1959), Vol. I, p. 315; D. Amati, M. Ciafaloni, and G. Veneziano, Higher order gravitational deflection and soft bremsstrahlung in planckian energy superstring collisions, Nucl. Phys. B347, 550 (1990); D. Amati, M. Ciafaloni, and G. Veneziano, Towards an S-matrix description of gravitational collapse, J. High Energy Phys. 02 (2008) 049; E. Laenen, G. Stavenga, and C. D. White, Path integral approach to eikonal and nextto-eikonal exponentiation, J. High Energy Phys. 03 (2009) 054; S. Melville, S. G. Naculich, H. J. Schnitzer, and C. D. White, Wilson line approach to gravity in the high energy limit, Phys. Rev. D 89, 025009 (2014); R. Akhoury, R. Saotome, and G. Sterman, High energy scattering in perturbative quantum gravity at next to leading power, arXiv:1308.5204; P. Di Vecchia, A. Luna, S. G. Naculich, R. Russo, G. Veneziano, and C. D. White, A tale of two exponentiations in $\mathcal{N}=8$ supergravity, Phys. Lett. B 798, 134927 (2019); M. Kulaxizi, G. S. Ng, and A. Parnachev, Subleading eikonal, AdS/CFT and 'double stress tensors, J. High Energy Phys. 10 (2019) 107; Z. Bern, H. Ita, J. ParraMartinez, and M. S. Ruf, Universality in the classical limit of massless gravitational scattering, arXiv:2002.02459; S. Abreu, F. Febres Cordero, H. Ita, M. Jaquier, B. Page, M. S. Ruf, and V. Sotnikov, The Two-Loop Four-Graviton Scattering Amplitudes, arXiv:2002.12374.

[69] M. Ciafaloni and D. Colferai, Rescattering corrections and self-consistent metric in Planckian scattering, J. High Energy Phys. 10 (2014) 085.

[70] Z. Bern, A. Luna, R. Roiban, C. H. Shen, and M. Zeng (to be published).

[71] V. B. Berestetskii, E. M. Lifshitz, and L. P. Pitaevskii, Quantum Electrodynamics (Pergamon Press, Oxford, 1982).

[72] I. B. Khriplovich and A. A. Pomeransky, Equations of motion of spinning relativistic particle in external fields, Zh. Eksp. Teor. Fiz. 113, 1537 (1998) [J. Exp. Theor. Phys. 86, 839 (1998)]; I. B. Khriplovich and A. A. Pomeransky, Equations of motion of spinning relativistic particle in external fields, Surv. High Energy Phys. 14, 145 (1999); I. B. Khriplovich and A. A. Pomeransky, Gravitational interaction of spinning bodies, center-of-mass coordinate 
and radiation of compact binary systems, Phys. Lett. A 216, 7 (1996).

[73] W. D. Goldberger and A. K. Ridgway, Radiation and the classical double copy for color charges, Phys. Rev. D 95, 125010 (2017); W. D. Goldberger, S. G. Prabhu, and J. O. Thompson, Classical gluon and graviton radiation from the bi-adjoint scalar double copy, Phys. Rev. D 96, 065009 (2017); W. D. Goldberger and A. K. Ridgway, Bound states and the classical double copy, Phys. Rev. D 97, 085019 (2018); D. Chester, Radiative double copy for EinsteinYang-Mills theory, Phys. Rev. D 97, 084025 (2018); W. D. Goldberger, J. Li, and S. G. Prabhu, Spinning particles, axion radiation, and the classical double copy, Phys. Rev. D 97, 105018 (2018); C. H. Shen, Gravitational radiation from color-kinematics duality, J. High Energy Phys. 11 (2018) 162; Y. F. Bautista and A. Guevara, On the double copy for spinning matter, arXiv:1908.11349.

[74] W. B. Kilgore, One-loop integral coefficients from generalized unitarity, arXiv:0711.5015; S. Badger, Direct extraction of one-loop rational terms, J. High Energy Phys. 01 (2009) 049.

[75] J. Vines, D. Kunst, J. Steinhoff, and T. Hinderer, Canonical Hamiltonian for an extended test body in curved spacetime: To quadratic order in spin, Phys. Rev. D 93, 103008 (2016).

[76] H. Johansson and A. Ochirov, Double copy for massive quantum particles with spin, J. High Energy Phys. 09 (2019) 040; Y. F. Bautista and A. Guevara, On the double copy for spinning matter, arXiv:1908.11349.

[77] I. B. Khriplovich and M. E. Pospelov, Magnetic quadrupole moment of W boson in Kobayashi-Maskawa model, Nucl. Phys. B420, 505 (1994).

[78] V. Rahal and H. Ren, The multipole expansion of conserved tensor currents and the number of form-factors, Phys. Rev. D 41 (1990) 1989.

[79] M. Fierz and W. Pauli, On relativistic wave equations for particles of arbitrary spin in an electromagnetic field, Proc. R. Soc. A 173, 211 (1939); M. Fierz and G. Pasa, On the relativistic theory of force-free particles with any spin, arXiv: 1704.00662.

[80] L. P. S. Singh and C. R. Hagen, Lagrangian formulation for arbitrary spin. 1. The boson case, Phys. Rev. D 9, 898 (1974).

[81] J. R. Klauder and B.-S. Skagerstam, Coherent States: Applications in Physics and Mathematical Physics (World Scientific, Singapore, 1985), https://doi.org/10.1142/0096.

[82] S. Deser and A. Waldron, Inconsistencies of massive charged gravitating higher spins, Nucl. Phys. B631, 369 (2002).

[83] A. Cucchieri, M. Porrati, and S. Deser, Tree level unitarity constraints on the gravitational couplings of higher spin massive fields, Phys. Rev. D 51, 4543 (1995); I. Cortese,
R. Rahman, and M. Sivakumar, Consistent non-minimal couplings of massive higher-spin particles, Nucl. Phys. B879, 143 (2014).

[84] I. Giannakis, J. T. Liu, and M. Porrati, Massive higher spin states in string theory and the principle of equivalence, Phys. Rev. D 59, 104013 (1999).

[85] L. L. Foldy and S. A. Wouthuysen, On the Dirac theory of spin $1 / 2$ particle and its nonrelativistic limit, Phys. Rev. 78 (1950) 29.

[86] M. B. Green, J. H. Schwarz, and E. Witten, in Superstring Theory. Vol. 1: Introduction, Cambridge Monographs On Mathematical Physics (Cambridge University Press, Cambridge, England, 1987), p. 469.

[87] G. Passarino and M. J. G. Veltman, One loop corrections for e+ e- annihilation into mu+ mu- in the Weinberg model, Nucl. Phys. B160, 151 (1979).

[88] R. K. Ellis and G. Zanderighi, Scalar one-loop integrals for QCD, J. High Energy Phys. 02 (2008) 002.

[89] W. Beenakker and A. Denner, Infrared divergent scalar box integrals with applications in the electroweak Standard Model, Nucl. Phys. B338, 349 (1990).

[90] W. E. Caswell and G. P. Lepage, Effective Lagrangians for bound state problems in QED, QCD, and other field theories, Phys. Lett. 167B, 437 (1986); G. T. Bodwin, E. Braaten, and G. P. Lepage, Rigorous QCD analysis of inclusive annihilation and production of heavy quarkonium, Phys. Rev. D 51, 1125 (1995); , Erratum, Phys. Rev. D 55, 5853 (1997).

[91] E. Barausse, E. Racine, and A. Buonanno, Hamiltonian of a spinning test-particle in curved spacetime, Phys. Rev. D 80, 104025 (2009).

[92] See Supplemental Material at http://link.aps.org/ supplemental/10.1103/PhysRevD.104.065014 for the coefficients of the Hamiltonian and amplitudes are provided in the Mathematica text ancillary file, coefficients.m. The results of momentum impulse and spin kick in a scattering event is given in the file DeltaP_DeltaS.m.

[93] T. Damour, Coalescence of two spinning black holes: An effective one-body approach, Phys. Rev. D 64, 124013 (2001).

[94] T. Damour, P. Jaranowski, and G. Schaefer, Effective one body approach to the dynamics of two spinning black holes with next-to-leading order spin-orbit coupling, Phys. Rev. D 78, 024009 (2008); E. Barausse and A. Buonanno, An improved effective-one-body Hamiltonian for spinning black-hole binaries, Phys. Rev. D 81, 084024 (2010); M. Khalil, J. Steinhoff, J. Vines, and A. Buonanno, Fourth postNewtonian effective-one-body Hamiltonians with generic spins, arXiv:2003.04469.

[95] T. Damour, Classical and quantum scattering in postMinkowskian gravity, Phys. Rev. D 102, 024060 (2020). 